\title{
2019 Lumbar Spine Research Society Annual Scientific Meeting Oral and Poster Presentations Chicago, Illinois, April 4-5, 2019
}

\author{
(DOI: 10.3171/2019.4.SPINE.LSRS2019abstracts)
}

Disclaimer: The Journal of Neurosurgery Publishing Group (JNSPG) acknowledges that these abstracts are published as submitted and did not go through JNSPG's peer-review or editing process.

\section{Paper 01. Risk Factors Associated with Development of Urinary Retention Following Posterior Lumbar Spinal Fusion}

Jeremy Mormol, $B S^{l}$, Bryce Basques, $M D^{l}$, Kevin Alter, $B S^{2}$, Jannat Khan, $B S^{2}$, Philip Louie, $M D^{l}, E d w a r d$ Goldberg, $M D^{2}$, Matthew Colman, $M D^{3}$, Howard An, $M D^{I}$

${ }^{I}$ Rush University Medical Center, Chicago, IL, ${ }^{2}$, Chicago, IL, ${ }^{3}$ Rush University, Chicago, IL

Background/Introduction: Postoperative urinary retention (POUR) is common following lumbar spinal fusion procedures. Most risk factors that have been identified are nonmodifiable and therefore not useful for the prevention of this complication. The present study aims to further investigate characteristics associated with increased occurrence of POUR.

Materials/Methods: A retrospective cohort study was performed using consecutive patients who underwent posterior lumbar laminectomy and fusion at an academic institution between 2011 and 2018. Patient demographic and comorbidity information, operative characteristics and postoperative characteristics were collected. Patients with a history of urologic problems other than BPH or had a prior history of POUR were excluded. The standard protocol for all lumbar fusion patients at the institution is to document the first three post-void residuals (PVRs) using a bladder scan, with POUR necessitating straight catheterization defined as a PVR greater than $400 \mathrm{ml}$. All variables were tested for association using a multivariate regression model created in backwards stepwise fashion, where variables with the highest $\mathrm{p}$ value were sequentially removed until only those with p-values $<0.200$ remained in the model. The threshold for statistical significance was set at $\mathrm{p}<0.05$.

Results: 814 patients were included in this study. Average age was 58 years, average BMI was 30.89 and $52.95 \%$ of patients were female. Out of the total cohort, $138(16.9 \%)$ had urinary retention requiring at least one straight catheterization. In the final multivariate model, prior diagnosis of BPH (Odds Ratio [OR] 3.46; $\mathrm{p}=<0.001)$, and American Society of Anesthesiologists (ASA) class 3 or greater (OR 1.58; $\mathrm{p}=0.043)$ were the best preoperative predictors of POUR, while prior history of lumbar spine surgery (OR $0.56 ; p=0.022)$ was associated with decreased rates of POUR. (Table 1) Glycopyrrolate use (OR 2.06; $\mathrm{p}=0.01$ ) and postoperative urinary tract infection (UTI) [OR $5.72 \mathrm{p}=0.004]$ were additionally associated with POUR.

Discussion/Conclusion: In a series of 814 posterior lumbar fusion patients, BPH, ASA 3 or greater, use of glycopyrrolate and postoperative UTI were independent predictors of POUR. The use of glycopyrrolate in anesthesia reversal is potentially modifiable and provides an attractive target for limiting the occurrence of this common, but impactful, complication following lumbar fusion. 


\title{
Paper 02. Beta-Lactam Antibiotic Surgical Prophylaxis is Safe in Patients with Self-Reported Penicillin Allergies: A Cohort Study of Lumbar Spine Surgery Patients
}

\author{
Andrew Pugely, $M D^{l}$, Joseph Laratta, $M D^{2}$, Alan Shamrock, $M D^{3}$, Piyush Kalakoti, $M D^{4}$ \\ ${ }^{1}$, New York, NY, ${ }^{2}$ The Spine Hospital at Columbia University, New York, NY, ${ }^{3}$ University of Iowa, Iowa \\ City, Iowa, ${ }^{4}$ University of Iowa, Iowa City, IA
}

Background/Introduction: The use of first-generation cephalosporin, cenfazolin, for antibiotic surgical prophylaxis in spine surgery remains strongly recommended. Given the distant cross-reactivity, patients with Penicillin (PCN) allergies are often not administered cephalosporins, but instead given less optimal alternative antibiotics. The reliability, however, of patient-reported penicillin allergies has been disputed. The purpose of this study was to determine the safety of administering beta-lactam antibiotics in those reporting a PCN.

Materials/Methods: A retrospective review of pertinent medical records of all patients undergoing complex lumbar spinal surgery at the University of Iowa between 2015-2016 that were referred to the Drug Allergy Clinic was performed. Primary outcome measure was comparison of penicillin skin testing results with patient reported drug allergies to PCN and/or beta-lactam antibiotics. Descriptive statistics were utilized for data analysis/interpretation.

Results: Overall, twenty-nine patients undergoing lumbar spine surgery with reported PCN allergies were referred to a Drug Allergy Clinic for evaluation of optimal surgical antibiotic prophylaxis. Of these referrals, 19 completed appointments in the Allergy Clinic where they underwent penicillin skin testing (PST), a drug challenge to a beta-lactam antibiotic, and/or had no intervention depending on the history obtained. Penicillin skin testing was performed on 17 of 19 (89\%) patients. No testing was indicated in 2 patients as an allergy was ruled out by the history. A negative PST was noted in all 17 (100\%) patients tested, indicating no penicillin allergy. Cefazolin was deemed safe to use in all 19 patients (100\%) patients evaluated. Eighteen of the patients evaluated in the Allergy Clinic completed a lumbar surgery. Cefazolin in any surgical prophylaxis regimen was used in all 18 surgeries (100\%) without any adverse peri-operative reactions.

Discussion/Conclusion: Patients with a self-reported history of PCN allergies are highly unlikely to have a reaction to Beta-Lactam antibiotics administered as surgical prophylaxis. A strong consideration should be given towards routine use of cefazolin despite reported allergies. Referral to a Drug Allergy Clinic is an excellent option to optimize surgical antibiotic prophylaxis in those with self-reported allergies. 


\section{Paper 03. Risk Factors for Readmission and Complications in Obese and Severely Obese Patients Undergoing Elective Posterior Lumbar Spine Fusion}

Deeptee Jain, $M D^{I}$, Jeremy Shaw, $M D^{2}$, Vedat Deviren, $M D^{3}$, alan Zhang, $M D^{4}$, Sigurd Berven, $M D^{3}$ ${ }^{l}$ New York University, Apt 10D, New York, ${ }^{2}$ University of Pittsburgh, Pittsburgh, PA, ${ }^{3}$, San Francisco, CA, ${ }^{4}$ University of California, San Francisco, San Francisco, $C A$

Background/Introduction: Obesity is an important risk factor for complications after lumbar posterior spine fusion (PSF) and poses unique challenges regarding safety and quality of care. Nonetheless, this patient population is not well studied. The purpose of this study was to determine risk factors for readmission in this specific patient population.

Materials/Methods: This was a retrospective case control study. Patients age $>18$ undergoing lumbar PSF were identified using ICD-9 codes in State Inpatient Database. Independent analyses were performed for three separate groups of patients: patients were identified as obese using codes for obesity or BMI $>30$, but not morbid obesity or BMI $>40$, severely obese using codes for morbid obesity or BMI $>40$, and normal if they had none of the above codes nor codes for overweight or underweight. Data were queried for demographics, comorbidities, surgery characteristics, and 90-day hospital readmission. Logistic multivariate regression analysis was performed.

Results: We analyzed 24,349 obese, 9,835 severely obese patients and 257,986 normal patients. 90-day hospital readmission rates was $14.5 \%, 17.5 \%$, and $12.1 \%$ in the obese, severely obese, and normal groups, respectively. In the obese group, risk factors for readmission included female gender, Black/Hispanic race, Medicare/Medicaid insurance, anterior or lateral interbody fusion, $>3$ levels, cerebrovascular disease, chronic pulmonary disease, congestive heart failure, diabetes, myocardial infarction, peptic ulcer disease, peripheral vascular disease, renal disease, and mental health disease, In the severely obese group, risk factors for readmission included female gender, Medicare/Medicaid insurance, $>3$ levels, CHF, diabetes without and with chronic complication, hemi/paraplegia, mild liver disease, and renal disease. In the normal group, all studied variables were risk factors for readmission.

Discussion/Conclusion: Ninety-day hospital readmission rates in obese and severely obese patients undergoing elective lumbar PSF are high. Risk factors include demographic variables such as race and insurance status, as well as various comorbid conditions. Similar risk factors were seen in normal weight patients, however with less magnitude, suggesting a synergistic effect of obesity with other risk factors. This study provides valuable insight into modifiable risk factors that may be potential targets for preoperative optimization and guidance on risk adjustment in this unique patient population. 


\section{Paper 04. Declining Trend in Osteoporosis Screening and Management Following Vertebral Fractures - A National Analysis of Commercial and Medicare Advantage Claims}

Azeem Malik, MBBS ${ }^{l}$, Sheldon Retchin, $M D^{2}$, Frank Phillips, $M D^{3}$, Kaleigh Peters, MS, APRN, NP-C , Wendy $\mathrm{Xu}, \mathrm{PhD}^{2}$, Elizabeth $\mathrm{Yu}, \mathrm{MD}^{4}$, Safdar Khan, $M D^{5}$

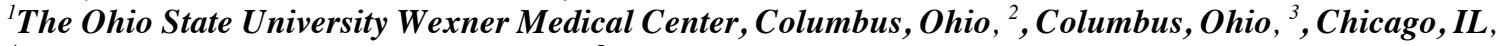
${ }^{4}$ Ohio State University, Columbus, Ohio, ${ }^{5}$, Columbus, $\mathrm{OH}$

Background/Introduction: Despite the increasing national incidence of osteoporosis, no study has evaluated the trends and rates of osteoporosis screening and associated medical management following thoracolumbar osteoporotic vertebral compression fractures (VCFs).

Materials/Methods: The 2008-2015Q3 Humana database was queried using ICD-9 diagnosis codes to identify patients with a primary osteoporotic VCF. Patients with a concurrent diagnosis of trauma/malignancy and a history of a fragility fracture in the year prior to the VCF were excluded to prevent overlap in treatment/screening rates. Apart from studying trends in the screening and medical management of osteoporosis, we also analyzed differences in the rates of second fragility fractures of vertebrae, hip, distal radius and proximal humerus between those patients who received anti-resorptive medication compared with those who did not.

Results: A total of 6,464 primary osteoporotic VCFs were retrieved from the database. Only $28.8 \%$ $(\mathrm{N}=1,860)$ patients received some form of anti-resorptive medication in the year following the VCF, with an average cost of treatment to be $\$ 1,511$ and median adherence/medication possession rate to be $69.5 \%$. An overall declining trend was noted in rates of osteoporosis management (from $37.9 \%$ to $25.9 \%$ ) between 2008 and 2015 . Only $36.7 \%$ patients received a DEXA scan in the year following the VCF. Risk factors associated with no prescription of anti-resorptive medication within 1 year of VCF were male gender (OR 1.17 [95\% CI 1.01-1.35]; p=0.027), history of CVA/stroke (OR 1.56 [95\% CI 1.08-2.32]; p=0.022), diabetes mellitus (OR 1.28 [95\% CI 1.04-1.58]; $\mathrm{p}=0.023$ ). Patients in the West vs. Midwest (OR 1.26 [95\% CI 1.04-1.51]; $\mathrm{p}=0.016$ ) and commercial insurance beneficiaries (OR 1.95 [95\% CI 1.08-3.52]; $\mathrm{p}=0.027$ ) were more likely to receive anti-resorptive medication. Patients on anti-resorptive medication vs. those not taking any medication were significantly less likely to receive a second fragility fracture (17.1\% vs. $43.3 \%$; $\mathrm{p}<0.001)$.

Discussion/Conclusion: The proportion of patients starting anti-resorptive medication within a year after a VCF remains low (28.8\%). Furthermore, a declining trend of anti-resorptive medication prescription was noted over time. Understanding this gap in care can allow providers to launch enhanced bone health/fracture prevention programs in their institutions to reduce the financial burden of osteoporotic fractures on the health-care system. 


\section{Paper 05. Local Mechanical Environment Plays a Role in Spinal Bone Mineral Density Measured by Quantitative Computed Tomography: A Study on Lumbar Lordosis}

Ichiro Okano, $M D^{l}$, Brandon Carlson, $M D, M P H^{l}$, Fabian Winter, $M D{ }^{l}$, Stephan Salzmann, $M D^{l}$, Toshiyuki Shirahata, $M D, P h D^{2}$, John Carrino, $M D, M P H^{l}$, Andrew Sama, $M D^{l}$, Frank Cammisa, $M D^{l}$, Federico Girardi, $M D^{3}$, Alexander Hughes, $M D^{l}$

${ }^{l}$ Hospital for Special Surgery, New York, New York, ${ }^{2}$ Hospital for Special Surgery, New York, New York, ${ }^{3}$, New york, New York

Background/Introduction: Patients with a degenerative spine condition have a different weight load distribution compared to healthy individuals. There have been some reports on the association between spinal balance parameters and regional bone mineral density (BMD), but the results are controversial.. The purpose of this study is to evaluate the relationship between spino-pelvic parameters and regional volumetric BMDs (vBMD) measured by QCT in the lumbo-sacral region among patients undergoing lumbar fusion surgery.

Materials/Methods: The data of consecutive patients undergoing posterior lumbar spinal fusion between 2014-2017 was reviewed. We included patients with preoperative computed tomography of the lumbarspine and standing whole spine radiographs. Patients with severe coronal imbalance, or any ongoing antiosteoporotic treatment were excluded. QCT measurements were conducted in L1 to S1 vertebral trabecular bone. The associations between each spino-pelvic sagittal parameters (SVA, LL, TK, PI, PT, SS) and vBMDs from L1 to S1 were evaluated

Results: 145 patients were included in the final analyses. Mean age ( \pm SD) was $65.4( \pm 11.7) .55 .2 \%$ of the patients were female. Mean vBMD in L1 $( \pm$ SD) was $118.3 \pm 37.4 \mathrm{mg} / \mathrm{cm} 3$. After adjusting by age, gender, race, and BMI, LL was negatively associated with vBMDs in all levels from L1-L5 (\% coefficient and Rsquare: L1: -0.442, 0.269; L2 -0.551, 0.297; L3 -0.611, 0.363; L4 -0.554, 0.228 ; L5 -0.431, 0.196), but not in S1. SS was negatively associated with vBMD only at L4 (\% coefficient -0.586). SVA, PI, PT, TK were not associated with vBMDs at any levels.

Discussion/Conclusion: We demonstrated that higher LL was associated with lower vBMDs in all levels. Our results suggest that BMD is affected not only by metabolic factors, but also by the mechanical environment. It is also implied that local mechanical forces have different loading effects on vertebral bodies that affect bone density. The effect of LL on vBMD was most significant at L3. L3 is usually located at the apex of the lumbar curve. Due to this anatomical characteristic, LL change might influence the weight load distribution and regional vBMD in L3 more than other lumbar vertebrae. 


\section{Paper 06. Clinical Level of Evidence Presented at the Lumbar Spine Research Society (LSRS) Annual Meeting Over 10 Years (2008-2017): A Systematic Review}

Nicholas Eyrich, M.S. ${ }^{l}$, Bridger Rodoni, B.S. ${ }^{I}$, Rakesh Patel, M.D. ${ }^{2}$; Ilyas Aleem, MD, MSc, FRCSC ${ }^{l}$ ${ }^{I}$ University of Michigan, Ann Arbor, MI, ${ }^{2}$, Ann Arbor, MI

Background/Introduction: The Lumbar Spine Research Society (LSRS) is dedicated to advancing knowledge of the lumbar spine to promote evidence-based care. We sought to systematically review the level of clinical evidence presented at LSRS annual meetings from 2008 through 2017.

Materials/Methods: A total of 458 paper abstracts presented at LSRS annual meetings were independently assessed by two reviewers. Abstracts were excluded based on specific criteria resulting in only clinical studies being included for analyses. Reviewers designated a clinical level of evidence (LOE) to each included abstract from level I to level IV based on criteria set forth by the American Academy of Orthopaedic Surgeons (AAOS). Reviewer agreement was assessed using Cohens Kappa coefficient (k) and disagreements were subsequently discussed until a consensus was reached. Student's t-test was used to assess for differences in mean LOE grades. Chi-squared testing was used to assess nonrandom changes in level of evidence.

Results: A total of 299 abstracts met the inclusion criteria. Over the last ten LSRS meetings, $2.68 \%$ of the presentations were level I, $22.4 \%$ were level II, $37.1 \%$ were level III, and $37.8 \%$ were level IV. We found the average LOE from 2008 to 2017 to be 3.10 (median = 3). Additionally, 63.9\% presentations were Therapeutic Studies, $30.1 \%$ were Prognostic Studies, and 6.02\% were Diagnostic studies. When comparing the first five years (2008-2012) to the last five years (2013-2017), we observed a significant increase in Level II $(\mathrm{p}<0.05)$ and Level III $(\mathrm{p}<0.05)$ evidence along with a corresponding decrease in level IV evidence $(\mathrm{p}<0.01)$. The average LOE improved significantly from 3.28 (2008-2012) to 2.88 (2013-2017) $(\mathrm{p}<0.001)$.

Discussion/Conclusion: Emphasis on evidence-based medicine within spine surgery, specifically pertaining to the lumbar spine, has positively influenced the clinical level of evidence disseminated at LSRS annual meetings between 2008 and 2017. Continued focus on higher quality Level I studies is warranted. 


\section{Paper 07. Teriparatide Treatment Improves Bone Quality in the Lumbar Spine Out of Proportion to DEXA Changes}

Anthony Mikula, $M D^{1}$, Ross Puffer, $M D^{2}$, Jeffrey St. Jeor, BS ${ }^{2}$, Jeremy Fogelson, MD ${ }^{3}$, A. Noelle Larson, $M D^{2}$, Ahmad Nassr, MD ${ }^{1}$, Bradford Currier, M.D. ${ }^{4}$, Mohamad Bydon, M.D. ${ }^{4}$, Michael Yaszemski, MD, $P h D^{2}$, Benjamin Elder, $M D, P h D^{3}$

${ }^{I}$ Mayo Clinic, Rochester, $M N,{ }^{2}$, Rochester, Minnesota, ${ }^{3}$ Mayo Clinic, Rochester, Minnesota, ${ }^{4}$, Rochester, MN

Background/Introduction: Teriparatide has been used to improve bone quality when considering spinal fusion surgery in patients with osteoporosis. Hounsfield units (HU), a standardized measurement for radiodensity, can be obtained on routine computed tomography (CT) scans, while dual energy x-ray absorptiometry (DEXA) is the standard test for bone density. The purpose of this study was compare changes in bone quality in the lumbar spine between Hounsfield Unit measurements and dual energy x-ray absorptiometry (DEXA) following Teriparatide treatment.

Materials/Methods: A retrospective chart review was performed from 1997 to 2018 across all campuses at our institution. We identified patients who had been treated with at least 6 months of Teriparatide with a CT scan and DEXA scan before and after therapy. HU were measured from L1-L4 with three measurements per vertebral body.

Results: Fifty-two patients (46 women, six men) were identified for analysis with an average age of 66.5 years who underwent an average of $20.9 \pm 6.5$ months of Teriparatide therapy. Average ( \pm standard deviation) HU improvement throughout the lumbar spine (L1-L4) was from $109.8 \pm 53$ to $133.9 \pm 61$ with a p-value of 0.039 and a 95\% CI from 1.2 to 46.1. Each individual lumbar level also had an average improvement in HU but only L1 was statistically significant (L1 improved from 112.4 to 139.2 with a pvalue of 0.013). Based on DEXA results, BMD (measured in $\mathrm{g} / \mathrm{cm} 2$ ) in the lumbar spine improved from $0.85 \pm 0.21$ to $0.93 \pm 0.21$ with a p-value of 0.044 . Lumbar spine t-scores improved from $-2.4 \pm 1.5$ to $-1.7 \pm 1.5$ with a p-value of 0.03 . Average femoral BMD only improved from $0.65 \pm 0.17$ to $0.67 \pm 0.17$ with a p-value of 0.43 . Femoral t-scores improved from $-2.5 \pm 1.1$ to $-2.3 \pm 1.0$ with a p-value of 0.31 .

Discussion/Conclusion: Teriparatide treatment improved BMD based on HU and DEXA in the lumbar spine, without a change in femoral BMD. The $22 \%$ improvement in HU out of proportion to the DEXA results supports some surgeons' subjective sense that intraoperative bone quality following Teriparatide treatment is better than indicated by DEXA results, particularly in comparison to the femoral BMD. 


\title{
Paper 08. Impact of Certificate-of-Need (CON) State Laws on Costs and Outcomes following Elective Posterior Lumbar Fusions
}

\author{
Azeem Malik, $M B B S^{l}$, Sheldon Retchin, $M D^{2}$, Wendy Xu, $P h D^{2}$, Safdar Khan, $M D^{3}$ \\ ${ }^{1}$ The Ohio State University Wexner Medical Center, Columbus, Ohio, ${ }^{2}$, Columbus, Ohio, ${ }^{3}$, Columbus, \\ $\mathrm{OH}$
}

Background/Introduction: Certificate-of-Need (CON) state laws have been a major mandate of healthpolicies aimed at forming centralized health planning as a key-core of the US health-care system. Though the federal CON mandate was repealed in 1986, several states still continue to use it as a means of limiting health-care resource utilization while attempting to provide quality care in high-volume facilities. The current study aimed to assess whether elective posterior lumbar fusions (PLFs) performed in CON vs. No CON states had lower utilization rates, lower costs, and better quality of care.

Materials/Methods: The 2005-2014 100\% Medicare Standard Analytical File was queried to identify patients undergoing elective 1-to-2 level PLF. States were categorized into 'CON' and 'No CON' based on existing laws. For each group (CON vs. No CON), differences in per-capita utilization, 90-day reimbursements and proportion of high-volume facilities ( $>150$ procedures/year) vs. low-volume facilities ( $\leq 50$ procedures/year) have been reported. Multi-variate analyses were used analyze 90 -day complication/readmission rates between the two groups.

Results: A total of 188,687 patients underwent an elective 1-to-2 level PLF in a CON state and 167,642 patients received the procedure in a No CON state from 2005 to 2014. The average per capita utilization of PLFs was lower in CON states as compared to No CON states (14.5 vs. 15.4 per 10,000 population; $\mathrm{p}<0.001)$. Average 90-day re-imbursements between CON and No CON states differed by a small amount $(\$ 22,115$ vs. $\$ 21,802)$. CON states had higher proportion of high-volume facilities (CON vs. No CON $40.9 \%$ vs. $29.9 \%$; $\mathrm{p}<0.05)$ and lower proportion of low-volume facilities (CON vs. No CON $-37.2 \%$ vs. $45.0 \% ; \mathrm{p}<0.05$ ). PLFs performed in CON states had a slightly lower odds of 90-day complications (OR 0.97 [95\% CI 0.96-0.99]; $\mathrm{p}<0.001$ ) and readmissions (OR 0.95 [95\% CI 0.93-0.97]; $\mathrm{p}<0.001$ ).

Discussion/Conclusion: While the presence of CON laws was associated with lower utilization of elective 1-to-2 level PLFs, and a greater presence of high-volume facilities, the mandate's effect on enhancing quality-of-care, by reduction of 90-day readmissions and 90-day complications is minimally significant. Future research, involving other surgical sub-specialties, is needed before health-policy makers may consider the removal or alteration of these laws. 


\title{
Paper 09. Reoperation Rates After Single-Level Lumbar Discectomy in the Military Health System
}

\author{
Melvin Helgeson, MD; Scott Wagner, MD, Donald Fredericks, MD, Donald Colontonio, MD, Michael \\ Elsenbeck, MD, Daniel Christensen, MD, Clark Cady, MD \\ Walter Reed National Military Medical Center, Bethesda, MD
}

Background/Introduction: Despite advances in surgical techniques, rates of recurrent lumbar disc herniation(LDH) range from 5-24\%, representing the most common cause of surgical failure and subsequent reoperation. Our goal was to determine the reoperation rate, time to reoperation and type of revision procedures performed after single-level discectomy for LDH within the military health system.

Materials/Methods: Using CPT codes to capture lumbar MD procedures, 3999 patients undergoing primary single level microdiscectomy for lumbar disc herniation were identified between October 2012 and November 2017. Demographic data, as well as number and type of revision surgical intervention were investigated. Rates of subsequent procedures involving instrumented fusion and complications were recorded.

Results: We identified 415 patients $(10.3 \%)$ that required a revision surgery for a same-level reherniation. Of these patients requiring revision surgery at the same level of index procedure, $9.3 \%$ required more than one revision surgery. The majority of first time revision procedures consisted of revision MD (290/415, $69.9 \%$ ); however 125 patients (30.1\%) underwent single- or multi-level fusion for definitive treatment at time of first revision. Of the 40 patients requiring more than one revision, $21(52.5 \%)$ eventually required a fusion procedure, which was significantly higher than patients having only one revision $(\mathrm{p}=<0.0001)$. Overall, 146 patients $(35.2 \%)$ who underwent additional surgical treatment for reherniation of a single level MD required fusion at some timepoint. There were 21 complications in 18 patients $(5 \%)$ following revision surgery for first-time reherniation: incidental durotomy was the most common complication, accounting for $6(28.5 \%)$ of the complications.

Discussion/Conclusion: In our retrospective study of 3999 patients undergoing single level microdiscectomy for lumbar disc herniation, we identified 415 patients $(10.4 \%)$ undergoing subsequent reoperation for a recurrent $\mathrm{LDH}$ at the same level. Within this group, $9.6 \%$ would require two or more reoperations, and $52.5 \%$ of these patients would ultimately undergo a lumbar arthrodesis at the same level as the initial disc herniation. Our findings characterize the lumbar disc reherniation population in the military, with major implications for the prognosis and treatment strategy of these commonly-treated injuries. 


\section{Paper 10. Patient Outcomes Following Short-Segment Lumbar Fusion Are Not Affected by PI-LL Mismatch}

Srikanth Divi, M.D. ${ }^{l}$, Dhruv Goyal, BA ${ }^{l}$, Matthew Galetta, BA ${ }^{l}$, Kristen Nicholson, PhD ${ }^{l}$, Mark Kurd, $M D^{I}$, Greg Anderson, $M D^{2}$, Alan Hilibrand, $M D^{2}$, Alexander Vaccaro, $M D$ PhD MBA ${ }^{3}$, Chris Kepler, MD $M B A^{4}$, Gregory Schroeder, M.D. ${ }^{2}$

${ }^{1}$, Philadelphia, PA, ${ }^{2}$ Rothman Institute, Thomas Jefferson University Hospital, Philadelphia, PA, ${ }^{3}$ Rothman Institute, Philadelphia, PA, ${ }^{4}$, Philadelphia , PA

Background/Introduction: Mismatch between pelvic incidence (PI) and lumbar lordosis (LL) has been emphasized as a reason for poor outcomes in deformity surgery. However, this relationship is less clear in patients undergoing short segment lumbar fusions for degenerative lumbar disease.

Materials/Methods: A retrospective review was conducted at a single center, academic hospital. Patients undergoing one or two level lumbar fusion were retrospectively identified. Patients with a history of previous surgery, infection, tumor, or trauma and those with less than one year follow-up were excluded. Patients were separated into two groups based on postoperative PI-LL mismatch $\leq 10^{\circ}$ (NM) or PI-LL mismatch $>10^{\circ}(\mathrm{M})$. Outcomes including the PCS-12, MCS-12, ODI, VAS back and leg scores were analyzed. Absolute PROM scores, the recovery ratio and the percent of patients achieving MCID between groups were compared and a multiple linear regression analysis was performed.

Results: A total of 352 patients were included, with 71 patients in the no mismatch (NM) group, and 303 patients in the mismatch (M) group. There was a significant difference in pelvic tilt (PT), PI, LL, and PI-LL between the two groups at baseline and postoperatively $(\mathrm{p}<.001)$. Patients in the M group started with more PI-LL mismatch compared to the NM group $\left(22.1^{\circ}\right.$ vs. $\left.7.7^{\circ}, \mathrm{p}<.001\right)$ and this difference increased postoperatively $\left(24.3^{\circ}\right.$ vs. $\left.2.2^{\circ}, \mathrm{p}<.001\right)$. There were no differences between the two groups in terms of preoperative or postoperative outcome scores. Recovery ratio analysis shows that both groups improved to a similar amount postoperatively $(\mathrm{p}>.05)$ and there was no difference between the proportions of patients achieving MCID for both groups ( $\mathrm{p}>.05$ ). In addition, having a PI-LL mismatch postoperatively was not found to be an independent predictor of any outcome score on multivariate analysis.

Discussion/Conclusion: The results of this study show that patients that had a PI-LL mismatch preoperatively continued to have a significant PI-LL mismatch postoperatively. However, there were no differences in patient outcomes compared to patients without a mismatch. These findings suggest that while correction of spinopelvic parameters is an important consideration in deformity surgery, it may not as in surgery for lumbar degenerative disease. 


\section{Paper 11. Healthcare Utilization and Costs in the Two Years Prior to a Single-Level Posterior Lumbar Fusion: What Happens Before Surgery?}

Azeem Malik, $M B B S^{l}$; Nikhil Jain, $M D^{2}$, Sohrab Virk, $M D^{3}$, Frank Phillips, $M D^{4}$, Safdar Khan, $M D^{3}$ ${ }^{l}$ The Ohio State University Wexner Medical Center, Columbus, Ohio, ${ }^{2}$ Ohio State University, Columbus, Ohio, ${ }^{3}$, Columbus, $\mathrm{OH},{ }^{4}$, Chicago, IL

Background/Introduction: There is little data with regards to pre-operative costs incurred prior to a single level posterior lumbar fusion in the 2 years preceding the surgery. We sought to collate data using a national database to determine healthcare resource utilization prior to a single-level posterior lumbar fusion.

Materials/Methods: The PearlDiver Humana Database was queried for active records of patients undergoing primary single-level posterior lumbar fusions for degenerative spine pathology using CPT and ICD codes. Pre-operative healthcare utilization was categorized into Procedures (laminectomies/decompressions), office visits, physical therapy, radiology, injections (facet blocks/rhizotomies/epidurals), pain medications (NSAIDS and opioids) and muscle relaxants. Healthcare utilization categories were cross-referenced with concurrent diagnoses for degenerative spine pathology to ensure relevant billing records were retrieved. Per patient average reimbursements (PPAR) were calculated for each healthcare resource category with regard to specified pre-operative time periods $(0-3$ months, $0-6$ months, 0-1year and 0-2years).

Results: Records of 10,281 patients, Commercial(Comm) $=1408$ and Medicare Advantage(MA)=8873, undergoing primary single-level lumbar fusions were retrieved. Overall cost for spine-related care in the 2 years preceding surgery was $\$ 31,965,233$ (\$3,109/patient) and $\$ 9,858,501$ (\$7001/patient) in MA and Commercial beneficiaries respectively. Overall 2-year PPARs for each resource category were:- Office visits (MA $=\$ 888 ; \mathrm{C}=\$ 1133$ ); Physical Therapy $(\mathrm{MA}=\$ 554 ; \mathrm{Comm}=\$ 711)$, Radiology (MA=\$936; Comm $=\$ 2402)$, Injections ( $M A=\$ 1285$, Comm $=\$ 3055)$, Pain Meds $(M A=\$ 504$; Comm $=\$ 705)$, Muscle Relaxants(MA $=\$ 106 ;$ Comm $=\$ 137)$, Opioids $(\mathrm{MA}=\$ 206 ; \mathrm{Comm}=\$ 282)$ and Procedures $(\mathrm{MA}=\$ 6,904$; $\mathrm{Comm}=\$ 10,395)$. A high healthcare resource utilization was seen within the last 3-6 months prior to surgery with an average $35 \%-57 \%$ of overall PPAR/each category being accounted for in the last six months. Of note, $46.3 \%$ (MA)/49.5\%(Comm) of the overall PPAR for injections was accounted for in the last three months and $41.3 \%(\mathrm{MA}) / 41.9 \%(\mathrm{Comm})$ of the overall PPAR for Opioids were accounted for in the last six months.

Discussion/Conclusion: We observed a high utilization of spine-related care in the three to six months prior to a single level posterior fusion for both Commercial and Medicare Advantage enrollees. As we move towards value based payment models, judicious use of non-surgical interventions with proven benefit, particularly in patients that ultimately require surgical intervention, will be required to optimize cost-effectiveness. 
Paper 12. Epidural Steroid Injections Show Little Relationship with Improved Clinical Outcomes in both Operative and Non-operative Management of Degenerative Spondylolisthesis

Michael Gerling, $M D^{I}$, Cole Bortz, $B A^{2}$, Haddy Alas, $B A^{3}$, Avery Brown, $B S^{2}$, Katherine Pierce, $B A^{2}$, Aaron Buckland, $M D^{4}$, Themistocles Protopsaltis, $M D^{4}$, Jon Lurie, $M D, M S^{5}$, Wenyan Zhao, PhD ${ }^{5}$, Peter Passias, $M D^{2}$

${ }^{l}$ NYU LMC School of Medicine, TriBeCa, NY, ${ }^{2}$, New York, NY, ${ }^{3}$ SUNY Downstate College of Medicine, Brooklyn, NY, ${ }^{4}$ Department of Orthopedic Surgery, NYU Langone Orthopedic Hospital, NYU Langone Health, Manhattan, NY, ${ }^{5}$, Lebanon, $\mathrm{NH}$

Background/Introduction: This study investigates the relationship between epidural steroid injection(ESI) and surgical crossover rates for degenerative spondylolisthesis patients from both randomized and concurrent observational cohorts of the Spine Patient Outcomes Research Trial(SPORT).

Materials/Methods: Included:surgical candidates $>18$ yrs with degenerative spondylolisthesis. Excluded:patients receiving ESI before enrollment. Those who received ESI within 3-months of enrollment(ESI) and those who did not(no-ESI) were compared for differences in baseline characteristics Outcomes at 1-year, 2-year, 3-year, and 4-year intervals following enrollment were assessed within operative and non-operative groups using longitudinal mixed-effect models with random subject intercept term to account for correlations between repeated measurements. Treatment comparisons were performed at follow-up intervals. Area under the curve analysis for all time-points assessed global significance of treatment.

Results: Included:266 patients(192 no-ESI, 74 ESI). Patient groups did not differ in age, sex, BMI, or comorbidity burden(all p>0.05).No-ESI had greater baseline SF-36 body-pain scores(38 $\pm 20 \mathrm{vs}$ $30 \pm 16, \mathrm{p}=0.002)$ and self-reported surgical preference(38\% vs $11 \%, \mathrm{p}<0.001)$. There were no differences in surgical rates within 4-years of enrollment between no-ESI and ESI patients(61\% vs 62\%,p=0.97). Surgical ESI and no-ESI patients did not differ in op-time, blood loss, rates of decompression, multilevel fusion, or levels decompressed(all p>0.05). Surgical ESI and no-ESI patients showed no differences in postoperative HRQL changes at any follow-up interval, or in 4 year average HRQL outcomes. Non-operative no-ESI patients showed greater 4-year average improvement in SF-36 body pain(no-ESI:17.8, ESI:7.8,p=0.004) and physical function(no-ESI:13.1, ESI:4.3,p=0.005) than non-operative ESI patients. These improvements in SF-36 body pain were greater for non-operative no-ESI patients at 1 -year $(\mathrm{p}=0.002)$ and 3 -

years $(\mathrm{p}=0.005)$; improvements in $\mathrm{SF}-36$ physical function were greater for non-operative no-ESI patients at 1 -year $(\mathrm{p}=0.030)$ and 2 -years $(\mathrm{p}=0.002)$. Of patients that were initially non-operative, there was no difference between ESI and no-ESI groups in surgical crossover rates(ESI:52\%, no-ESI:39\%,p=0.15). For operative patients, crossover rates to non-operative treatment did not differ between ESI and no-ESI groups(ESI:23\%, no-ESI: $16 \%$,p=0.38).

Discussion/Conclusion: For surgical degenerative spondylolisthesis patients, there was no relationship between ESI and improved clinical outcomes over a 4 year study. For non-operative patients, ESI was associated with inferior pain reduction through 3 years of follow-up; however, this was confounded by higher levels of baseline pain. ESI showed little relationship with surgical crossover. 
Paper 13. Spino-pelvic sagittal alignment analyses of multi-level lumbar spondylolysis versus monolevel lumbar spondylolysis

Bin Wang, $M B^{l}$; Xu Sun, M.D. ${ }^{l}$, Zezhang Zhu, M.D. ${ }^{2}$, Bangping Qian, M.D. ${ }^{2}$, Yong Qiu, M.D. ${ }^{3}$, Zhen Liu, $M . D{ }^{4}$, Qingshuang Zhou, $M . M^{5}$, Liang Xu, M.D. ${ }^{2}$, Xi Chen, $M D^{3}$, Changzhi Du, MD

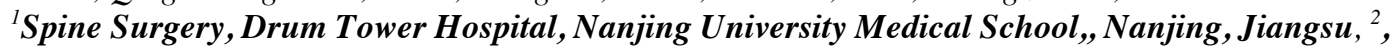
Nanjing, Jiangsu, ${ }^{3}$ Spine Surgery, Drum Tower Hospital, Nanjing University Medical School, Nanjing, Jiangsu, ${ }^{4}$, Nanjing, Jiangse, ${ }^{5}$ Drum Tower Hospital Clinical College of Nanjing Medical University, Nanjing, Jiangsu, ${ }^{6}, N A N J I N G, J I A N G S U$

Background/Introduction: Isthmic spondylolysis (IS) is a radiological defect or fracture of the pars interarticularis. Infrequent lumbosacral consecutive multi-level IS is revealed in only few cases and sagittal alignment of multi-level IS is not clear. The aim of this study is to compare demographic and radiographic data of IS with normal population and to compare these parameters between multi-level IS and mono-level IS.

Materials/Methods: We retrospectively reviewed 453 symptomatic isthmic spondylolysis patients who underwent surgical intervention at our single center between January 2007 and January 2018, and 158 asymptomatic volunteers were recruited. Basic radiographic parameters were measured, including slip parameters, pelvic parameters and sagittal alignment parameters. Pelvic incidence-lumbar lordosis (PI-LL) mismatch and the ratios of L4S1 segmental lordosis to LL (L4S1/LL), L5S1 segmental lordosis to LL (L5S1/LL) and pelvic tilt to PI (PT/PI) were also calculated to evaluate the relationship between spinopelvic parameters and a morphologic parameter characteristic of each individual.

Results: 51 (11.6\%) patients combined with multi-level IS (multi_IS) and 402 patients combined with mono-level IS (mono_IS). Pelvic parameters including PI, PT, SS and PT/PI were greater in IS patients compared to normal populations $(\mathrm{P}<0.001)$. There was no significant difference for PI and SS between multi_IS and mono_IS, but higher PT and PT/PI in multi_IS should be highlighted (P>0.05). LL and L4S1 segmental lordosis were higher in IS group compared with normal population $(\mathrm{P}<0.05)$, but L5S1 segmental lordosis was lowest in multi_IS group. Surprisingly, L4S1/LL and L5S1/LL were observed lower in IS group, which were particularly lowest in multi_IS (P<0.05). Furthermore, PI-LL showed a greater lack of lordosis in the multi_IS group than in the mono_IS group compared with normal populations $(\mathrm{P}<0.05)$. In comparison to normal population, larger $\mathrm{C} 7$ tilt degree and SVA were in IS, particularly for multi_IS $(\mathrm{P}<0.05)$. Functional and clinical outcomes of mono_IS were reported significantly worse than mono_IS $(\mathrm{P}<0.05)$

Discussion/Conclusion: Isthmic spondylolysis and spondylolisthesis had different sagittal alignment compared to asymptomatic volunteers, particularly in the lumbosacral region. Sagittal malalignment for multi_IS is characterized with trunk anterior tilt, insufficient of lumbosacral lordosis and pelvic retroversion. 


\section{Paper 14. Prediction of Complications, Readmission, and Revision Surgery Based on Duration of Preoperative Opioid Use: Analysis of Lumbar Fusion}

Nikhil Jain, $M D^{1}$, John Brock, BA ${ }^{2}$, Azeem Malik, $M B B S^{3}$, Frank Phillips, $M D^{4}$, Safdar Khan, $M D^{5}$ ${ }^{l}$ Ohio State University, Columbus, Ohio, ${ }^{2}$, Philadelphia, Pennsylvania, ${ }^{3}$, COLUMBUS, OHIO, ${ }^{4}$, Chicago, IL, ${ }^{5}$, Columbus, $\mathrm{OH}$

Background/Introduction: Preoperative opioid use results in adverse outcomes and higher costs after elective surgery. However, duration thresholds for higher risk are not entirely known. Therefore, the purpose of our study was to determine the number and duration of preoperative opioid prescriptions in order to estimate the risk of postoperative adverse events after lumbar fusion.

Materials/Methods: National insurance claims data (2007-Q3 2015) were used to identify primary 1 or 2level posterior lumbar fusions (PLFs) performed for degenerative disease. Longitudinal tracking of generic formulations of Schedule-II and III opioids taken by the oral route were used to record preoperative opioid use. The effect of preoperative opioid burden (naive, 3 months, $>3$ to 6 months, $>6$ months but stopped 3 months before surgery, and $>6$ months of continuous use) on the risks of various adverse outcomes was studied using Kaplan-Meier survival curves and Cox proportional hazards analysis with adjustment for demographic and clinical covariates. We chose specific adverse outcomes that are related to the effect of opioids on pain modulation, mobility, and immune function. All-cause and pain-related emergency department (ED) visits, falls, deep vein thrombosis and pulmonary embolism (DVT/PE), wound dehiscence and infection, systemic infection and sepsis, and hospital readmission, all within 90 days after the index surgery were studied. Revision surgery within 1 year after the index procedure was also studied.

Results: A total of 10,681 patients with 1 or 2-level PLFs were included. Preoperative opioid prescription for $>6$ months was associated with a higher risk of all-cause and pain-related ED visits, wound dehiscence/infection, and hospital readmission within 90 days as well as revision surgery within 1 year after PLF. Stopping the opioid prescription 3 months pre-operatively for chronic users resulted in a significant reduction in the risk of adverse outcomes (Fig. 1).

Discussion/Conclusion: Patients with a preoperative opioid prescription for up to 3 months before 1 or 2level lumbar fusion had a similar risk of adverse outcomes as opioid-naive patients. While $>6$ months of opioid use was associated with a higher risk of adverse outcomes, a 3-month prescription-free period before the surgery appeared to mitigate this risk for chronic users. 


\section{Paper 15. Impact of Preoperative Opioid Use on Postoperative Patient Reported Outcomes in Lumbar Spine Surgery Patients}

Joseph Snavely, $M D^{l}$, Joseph Weiner, $B S^{2}$, Daniel Johnson, $M D^{l}$, Wellington Hsu, M.D. ${ }^{3}$, Alpesh Patel, $M D, F A C S^{l}$

${ }^{I}$ Northwestern University, Chicago, IL, ${ }^{2}$ Northwestern University Department of Orthopaedic Surgery, Chicago , IL, ${ }^{3}$ Northwestern Memorial Hospital, Chicago, Illinois

Background/Introduction: As narcotic consumption increases in the United States, many spine surgery patients are already taking opioid pain medication prior to surgery. Preoperative opioid use is linked with adverse outcomes including increased complication rate and prolonged hospitalization. Our objective was to examine the impact of preoperative opioid use on patient reported outcomes following lumbar spine surgery. Outcomes data was collected with the Patient-Reported Outcomes Measurement Information System (PROMIS), a validated assessment tool in spine surgery patients.

Materials/Methods: 91 patients undergoing lumbar spine surgery at a single institution completed PROMIS Pain Interference (PI) scores preoperatively and at 3, 12, and 24 months postoperatively. 36 patients used opioid pain medication at the time of their preoperative office visit compared to 55 patients who did not. The differences between preoperative and postoperative PI scores of both groups were compared at all time points using a student's t-test. Post-surgical changes in PI scores were compared to a Minimally Important Difference (MID) of 8, as previously defined in this population.

Results: Preoperative opioid use showed significant effect on preoperative to postoperative change in PI score at all time points. At 3 months, patients in the non-opioid group had mean PI improvement of -9.44 versus -2.98 in the opioid group $(\mathrm{p}=0.024)$. At 12 months, patients in the non-opioid group had mean PI improvement of -11.5 versus -5.4 in the opioid group $(\mathrm{p}=0.041)$. The most significant difference was seen at 24 months as patients in the non-opioid group had mean PI improvement of -13.0 versus -4.9 in the opioid group ( $\mathrm{p}=0.014$ ). The non-opioid group achieved MID postoperatively at all time points, while the opioid group did not achieve MID at any time point.

Discussion/Conclusion: Preoperative opioid use showed a significant detrimental effect on postoperative improvement in PI scores and achievement of MID. Lumbar spine surgery patients who do not use opioids preoperatively show significant post-surgical improvement in PI scores compared to patients who use preoperative opioids. These improvements in postoperative outcomes are seen at all time points up to 2 years after surgery. These findings may be useful in determining appropriate surgical candidates and educating patients on post-surgical outcomes. 
Paper 16. Factors Associated with Opioid Prescription and Dependence Following Elective Lumbar Spine Surgery

Aidin Abedi, $M D^{l}$, Blake Formanek, $B A^{l}$, Jeffrey Wang, $M D^{2}$; Zorica Buser, PhD ${ }^{3}$

${ }^{l}$, Los Angeles, CA, ${ }^{2}$ Keck School of Medicine of USC, Los Angeles, CA, ${ }^{3}$ University of Southern California, Keck School of Medicine, Los Angeles, CA

Background/Introduction: Over-prescription of opioids during the past decades has led to an opioid crisis, with an estimate of two million chronic opioid users across the United States as of 2015. Orthopedic surgeons are among the top opioid prescribers and low back pain is one of the most common chronic pain conditions. Yet, there is little information about opioid prescription patterns and chronic use following spine surgery. The main objectives of this study were to identify the factors associated with opioid prescription in the early post-operative period, and to identify the risk factors for opioid dependence following elective lumbar spine surgery.

Materials/Methods: A national US insurance database (Humana) was queried for patients who had undergone lumbar surgery between 2008-2016. After inclusion of all procedures, patients with spinal trauma, infection and cancer were excluded. The minimum cut-off for opioid dependence was set at three months after the operation. The associations of demographics, pre-operative characteristics and surgical factors with post-operative opioid prescription and dependence were assessed using bivariate logistic regression.

Results: A total of 70556 patients with early post-operative opioid prescription and 49084 with postoperative opioid dependence were included. Men were less likely to receive opioids ( $O R=0.94, p<0.0001)$ and patients with a history of opioid prescription within a year prior to surgery had the largest odds of opioid prescription following surgery $(\mathrm{OR}=5.53, \mathrm{p}<0.0001)$. Other important predictors included previous opioid dependence/chronic use $(\mathrm{OR}=2.06)$, smoking $(\mathrm{OR}=1.64)$, mental health disorders $(\mathrm{OR}=1.54)$, alcoholism $(\mathrm{OR}=1.49)$, lateral fusion $(\mathrm{OR}=2.79)$ and grafted surgery $(\mathrm{OR}=1.67)($ all $\mathrm{p}$-values $<0.0001)$. While men were less likely to develop opioid dependence $(\mathrm{OR}=0.71)$, patients receiving long-acting opioids $(\mathrm{OR}=4.26)$, those undergoing spinal fusion $(\mathrm{OR}=2.98)$, and patients with a history of mental illnesses were among those with highest risks of opioid dependence (all p-values $<0.0001$ ).

Discussion/Conclusion: Our preliminary findings showed that patients with some well-known risk factors for opioid dependence were more likely to get exposed to opioids following elective lumbar surgery. Identified risk factors for opioid dependence can be integrated into risk reduction strategies. These finding should be confirmed using multivariate analysis since some of the identified factors are known to be present in clusters. 


\section{Paper 17. Accuracy of Pedicle Screw Placement using a Novel Augmented Reality and Artificial Intelligence Surgical Navigation System: A Cadaveric Study}

Karina Katchko, $B S^{l}$, Krzysztof Siemionow, $M D P h D^{2}$; Cristian Luciano, $P h D^{3}$

${ }^{1}$, Chicago, IL, ${ }^{2}$ Holosurgical SA, Warsaw, Masovia, ${ }^{3}$ Holosurgical SA, Warsaw, Mazovia

Background/Introduction: Minimally invasive spine surgery (MISS) has potential advantages over open procedures however, all currently available navigation systems force surgeons to look away from the field to verify instrument position. This requires development of complex coordination skills with a steep learning curve. Augmented, reality-based display of virtual spinal anatomy over patient spinal anatomy may serve as a more user-friendly navigation system. This study assessed the accuracy of lumbar percutaneous pedicle instrumentation using a novel, reality assisted surgical navigation system in a cadaveric model.

Materials/Methods: The navigation system consists of a projection screen that hovers over the surgical field and 3D goggles to visualize the virtual spine model collocated with the cadaver's internal anatomy. An optical tracker was used to determine the orientation of all components of the system. Using CT the system computed the spatial relationship between internal anatomy and registration arrays. Five cadaveric spine specimens were used for validation. A Jamshidi needle was introduced into the lumbar pedicles at the precise location identified by the navigation system as the entry point of each pedicle. A guide-wire, followed by a pedicle screw mounted on a navigated screwdriver were then inserted. After instrumentation $\mathrm{CT}$ was used to evaluate the $3 \mathrm{D}$ position of the probe or screw with respect to the actual pedicles.

Results: This novel surgical navigation system using augmented reality and artificial intelligence technology resulted in accurate identification and precise 3D visualization of lumbar pedicles directly in the surgical field while performing percutaneous pedicle instrumentation on cadavers. All attempted pedicle screws $(\mathrm{N}=20)$ were placed in acceptable position, there were no breaches on post-instrumentation $\mathrm{CT}$, and no aborted attempts.

Discussion/Conclusion: Accurate percutaneous pedicle screw placement provides optimal mechanical strength while improving patient safety and decreasing the morbidity of open spine procedures. The evaluated navigation system accurately identified and visualized the 3D position and orientation of multiple lumbar pedicles at all attempted levels. The virtual model of the internal anatomy was precisely displayed at the location of the actual spine in all tested scenarios. Further investigation of this technology may lead to better patient outcomes, reduce surgical time, and decrease the learning curve for MISS. 


\section{Paper 18. Comparative Effectiveness of Adjustable Lordotic Expandable versus Static Lateral Lumbar Interbody Fusion Devices: One Year Clinical and Radiographic Outcomes}

Yan Li, MD, PhD ${ }^{l}$, Richard Frisch, $M D^{2}$, James Towner, $M D^{3}$, Yan Li, PhD ${ }^{4}$, Jessica Riggleman, BS ${ }^{5}$, Charles Ledonio, $M D^{5}$

${ }^{l}$ Neurosurgery University of Rochester Medical Center School of Medicine and Dentistry, Rochester, NY, ${ }^{2}$ Southeastern Spine Institute, Mt Pleasant, SC, ${ }^{3}$ University of Rochester Medical Center, Rochester, NY, ${ }^{4}$ Univeristy of Rochester , NA, NA, ${ }^{5}$ Globus Medical, Inc., Audubon, PA

Background/Introduction: Use of large interbody spacers in minimally invasive lateral lumbar interbody fusion (MIS LLIF) offers favorable clinical and radiographic results. Static interbody spacers may cause iatrogenic endplate damage and implant subsidence due to forceful impaction and excessive trialing. Expandable interbody spacers with adjustable lordosis offer in situ expansion that may optimize endplate contact and maximize and maintain sagittal alignment correction until fusion occurs. This study compares the clinical and radiographic outcomes between patients treated with static and expandable interbody spacers with adjustable lordosis for MIS LLIF.

Materials/Methods: This is a multi-surgeon, retrospective, Institutional Review Board-exempt chart review of consecutive patients who underwent MIS LLIF at 1-2 contiguous level(s) using either a PEEK static (32) or titanium expandable spacer with adjustable lordosis (57). The mean differences of radiographic and clinical outcomes were compared from preoperative to postoperative 12-month follow-up. Statistical results were significant when $\mathrm{P}<0.05$.

Results: Mean improvement of VAS back and leg pain scores from preoperative to 6 and 12 months was significantly higher in the expandable group compared to the static group $(\mathrm{P}<0.05)$. Mean improvement of ODI scores from preoperative to 3,6, and 12 months was significantly higher in the expandable group compared to the static group $(\mathrm{P}<0.001)$. The expandable group had a significantly greater mean improvement in segmental lordosis from preoperative to 6 weeks, 3, 6, and 12 months $(\mathrm{P}<0.001)$. For disc height, the mean improvement from preoperative to 6 weeks and 3 months was more significant in the expandable group compared to the static group $(\mathrm{P}<0.05)$. In the expandable group, the mean improvement from preoperative to 6 weeks, 3 , and 6 months was significantly greater compared to the static group for neuroforaminal height $(\mathrm{P}<0.001)$. Subsidence was $0 \%$ in the expandable group and $32.4 \%(12 / 37)$ in the static group.

Discussion/Conclusion: This study showed significant positive clinical and radiographic outcomes for patients who underwent MIS LLIF using expandable interbody spacers with adjustable lordosis based on more significant changes in VAS and ODI scores, and radiographic parameters at 12-month follow-up. There was no subsidence in the expandable group, compared to a $32 \%$ subsidence rate in the static group. 


\section{Paper 19. Does L4/5 Pose Additional Neurologic Risk in Lateral Lumbar Interbody Fusion?}

Stephan Salzmann, $M D^{l}$, Toshiyuki Shirahata, $M D, P h D^{2}$, Ichiro Okano, $M D^{l}$, Oliver Sax, $M S^{3}$, Jingyan Yang, $M H S^{4}$, Jennifer Shue, $M S^{5}$, Andrew Sama, $M D^{l}$, Frank Cammisa, $M D^{l}$, Federico Girardi, $M D^{6}$, Alexander Hughes, $M D^{I}$

${ }^{l}$ Hospital for Special Surgery, New York, New York, ${ }^{2}$ Hospital for Special Surgery, New York, New York, ${ }^{3}$, New York, New York, ${ }^{4}$ Hospital for Special Surgery, New York, New York, ${ }^{5}$ Hospital for Special Surgery, New York, New York, ${ }^{6}$, New york, New York

Background/Introduction: Lateral lumbar interbody fusion (LLIF) at the L4/5 level is a controversial topic in the spine literature. Nerve-related motor deficits of the quadriceps and tibialis anterior muscle are especially of major clinical concern. However, clinical studies assessing the neurologic safety profile of LLIF at L4/5 show conflicting results. The aim of the current study was to compare the rate of nerverelated motor deficits in patients undergoing LLIF with and without L4/5 involvement.

Materials/Methods: Patients undergoing LLIF from 2006-2016 at a single academic institution were eligible for inclusion. Subjects with missing pre- or postoperative motor exams were excluded.

Demographic and clinical data were obtained. New postoperative motor deficits were defined as a decrease in motor strength in the tibialis anterior or quadriceps femoris muscle at first postoperative visit compared to preoperative baseline. Resolution of motor deficits at last follow-up was defined as return to preoperative baseline or recovery of full motor strength. Regression analysis was performed to examine the association of LLIF at L4/5 and the risk of new motor deficits.

Results: 872 patients treated with LLIF (mean age: 62.2 years, $57.2 \%$ female) met inclusion criteria. 543 patients underwent LLIF including the L4/5 level and 329 patients underwent LLIF without L4/5 involvement. The rate of new motor deficits at the 6 week postoperative visit in the L4/5 group was $13.1 \%$, which was significantly higher compared to the non-L4/5 group with $5.5 \%(\mathrm{p}<0.001)$. After adjusting for potential confounders in multivariate logistic regression models, L4/5 was still significantly associated with an increased risk for new motor deficit $(\mathrm{OR}=2.285 ; \mathrm{p}=0.008)$. At last follow-up (mean=16.6 months), $2.6 \%$ of the patients in the $\mathrm{L} 4 / 5$ group and $0.3 \%$ of the patients in the non-L $4 / 5$ group had remaining deficits.

Discussion/Conclusion: To our knowledge this is the largest study to date comparing postoperative motor deficits after LLIF in patients with and without involvement of L4/5. Our results are in line with previous studies which report an initial increased risk of new motor deficits for LLIF performed at L4/5. The majority of new motor deficits, however, was transient in nature and resolved over time. 
Paper 20. Does the operative microscope increase surgical site infections in lumbar decompression procedures?

Addisu Mesfin, $M D^{I}$, Ayodeji Jubril, $B S^{2}$; Adan Omar, $M D^{l}$

${ }^{l}$ University of Rochester, Rochester, $N Y,{ }^{2}$, Rochester, $N Y$

Background/Introduction: Lumbar decompressions for herniated discs or lumbar stenosis are common spine procedures. Some studies have raised the concern that drape contamination of the operative microscope may be an additional risk for surgical site infections. We hypothesize that the use of the operative microscope for lumbar decompression procedures does not increase infection rates. Our objective was to compare surgical site infection rates between patients undergoing lumbar discectomy with an operative microscope versus surgical loopes.

Materials/Methods: A retrospective cohort analysis was performed on patients undergoing lumbar spinal decompressions via microscopic assistance (MA) or loope assistance (LA) by two orthopaedic spine surgeons at a tertiary academic medical center. Patients treated from 11/2012 to 10/2016 were enrolled. Variables including age, sex, race, BMI, length of surgery, intra-operative complications, estimated blood loss and post-operative surgical site infections (SSI) within 30 days were collected.

Results: A total of 225 patients were included in the study. 63 patients underwent loupe assisted (LA) lumbar decompression, and 162 underwent microscope assisted (MA) lumbar decompression. There were $72 \mathrm{~F} / 90 \mathrm{M}$ in the MA group and $31 \mathrm{~F} / 33 \mathrm{M}$ in the LA group. The MA was significantly older 45.2 yersus 40.4 in LA, $\mathrm{p}=0.02$ and had a significantly higher BMI (30.64 versus $27.79, \mathrm{p}<0.002)$. SSI rates were not significantly different, MA $3.7 \%(6 / 162)$ and LA $7.9 \%(5 / 63), p=0.14$. The MA group had a significantly longer operative time (92 minutes versus 50 minutes, $\mathrm{p}<0.001)$. Dural tears rates were $3.1 \%$ in MA and $1.6 \%$ in LA, $\mathrm{p}=0.3$ and were associated with longer operative time in the MA group, 162.2 minutes versus 90.2 minutes, $\mathrm{p}<0.0001$.

Discussion/Conclusion: The use of the operative microscope had similar infection rates as loupe assisted microdiscectomies. In academic institutions, the operative microscope may allow more opportunities for residents or fellows to partake/assist in the procedure as compared to loupe assisted procedures. 


\section{Paper 21. Postoperative Recovery Kinetics of Adult Spinal Deformity Patients with Proximal Junctional Kyphosis}

Frank Segreto, $B S^{l}$; Peter Passias, $M D^{l}$, Renaud Lafage, $M S^{2}$, Christopher Ames, $M D^{3}$, Justin Smith, $M D^{4}$, Breton Line, $B S^{5}$, Frank Schwab, $M D^{I}$, Shay Bess, $M D^{5}$, Christopher Shaffrey, $M D^{6}$, Virginie Lafage, $P h D^{7}$ ${ }^{1}$, New York, NY, ${ }^{2}$ Hospital for Special Surgery, New York, New York, ${ }^{3}$ University of California San Fransisco, San Fransisco, CA, ${ }^{4}$ University of Virginia, Charlottesville, VA, ${ }^{5}$, Denver, Colorado, ${ }^{6}$ University of Virginia Medical Center, Charlottesville, VA, ${ }^{7}$ Hospital for Special Surgery, New York, $N Y$

Background/Introduction: Proximal junctional kyphosis (PJK), a common radiographic finding after long spinal fusions, is one of the greatest challenges facing spinal surgeons. Recovery profiles of ASD patients with varying degrees of PJK, relative to patients without PJK, are poorly understood. The purpose of this study was to investigate the unique recovery profiles of PJK patients compared to those without.

Materials/Methods: Operative ASD patients (Coronal scoliosis $\geq 20^{\circ}, \mathrm{SVA} \geq 5 \mathrm{~cm}, \mathrm{PT} \geq 25^{\circ}$, and $/$ or $\mathrm{TK} \geq 60^{\circ}$ ) $>18 \mathrm{y} / \mathrm{o}$, with complete baseline(BL) and $2 \mathrm{Y}$ follow up (f/u) HRQLs were included. PJK was defined as $\geq 10^{\circ}$ for the angle between the UIV inferior endplate and UIV +2 superior endplate. Patients were stratified into 3 groups: No PJK(N-PJK), PJK, and Severe PJK(S-PJK: $\geq 20^{\circ}$ ), and propensity matched by BL age, CCI, pelvic incidence, and HRQL scores. A novel method of area-under-the-curve(AUC) normalization generated normalized $\mathrm{HRQL}$ scores at $\mathrm{BL}$ and all $\mathrm{f} / \mathrm{u}$ intervals $(6 \mathrm{~W}, 1 \mathrm{Y}, 2 \mathrm{Y})$. AUC was calculated for each $\mathrm{f} / \mathrm{u}$, and total area was divided by cumulative f/u length, generating one number describing overall timeadjusted HRQL recovery (Integrated Health State-IHS).

Results: 240 patients were included(80 per group). Demographics(Age 60.2, BMI 27.6, CCI 1.7, Gender $78.7 \% \mathrm{~F}, 92.2 \%$ White, $3.9 \%$ Black) were similar among groups $(\mathrm{p}>0.05)$. BL deformity presentation: PT $23.8^{\circ}$, PI-LL $15.0^{\circ}$, SVA $65.0 \mathrm{~mm}$, TPA $22.5^{\circ}$. Surgical approaches were $0.8 \%$ anterior, $70.4 \%$ posterior, $28.7 \%$ combined; $41 \%$ reoperative, levels fused was 6.0 anteriorly, 11.3 posteriorly; op-time was $386.3 \mathrm{~min}$, EBL was 1702.5 , LOS was 8.1 days; all $p>0.05$ between groups. Following surgery, all groups exhibited improved $1 Y+2 Y$ ODI, SRS, NRS Back and Leg scores(all $\mathrm{p}<0.001)$. Standard analysis found groups to have similar HRQL scores at all f/u intervals, except PJK had significantly less W6 NRS Back(4.97 vs 3.83 vs $4.96, \mathrm{p}=0.036)$ and better $\mathrm{Y} 2 \mathrm{ODI}(29.9$ vs 24.2 vs $32.1, \mathrm{p}=0.041)$. After normalization, despite S-PJK trending towards worse ODI and SRS recovery, and N-PJK trending towards worse NRS Leg recovery, no significant differences in recovery patterns or IHS were observed(all $\mathrm{p}>0.05)$.

Discussion/Conclusion: Within our analysis, all patients displayed similar recovery profiles, regardless of PJK status. These objective recovery benchmarks can help plan expectations accordingly for varying postoperative f/u visits. 
Paper 22. The Consequence of Non-Operative Management in Adult Spinal Deformity: Long-Term Durability of Alignment in Non-Operative Adult Spinal Deformity Treatment Relative to Those Undergoing Correction

Peter Passias, $M D^{l}$, Cole Bortz, $B A^{l}$, Haddy Alas, $B A^{2}$, Katherine Pierce, $B A^{l}$, Avery Brown, $B S^{l}$, Christopher Shaffrey, $M D^{3}$; Michael Gerling, $M D^{4}$, Christopher Ames, $M D^{5}$, Virginie Lafage, PhD ${ }^{6}$, International Spine Study Group (ISSG), , -

${ }^{1}$, New York, NY, ${ }^{2}$ SUNY Downstate College of Medicine, Brooklyn, NY, ${ }^{3}$ University of Virginia Medical Center, Charlottesville, VA, ${ }^{4}$ NYU LMC School of Medicine, TriBeCa, NY, ${ }^{5}$ University of California San Fransisco, San Fransisco, CA, ${ }^{6}$ Hospital for Special Surgery, New York, NY, ${ }^{7}$, Littleton, Colorado

Background/Introduction: As compared to non-operative intervention, operative ASD-treatment has been associated with superior long-term alignment outcomes; however, the literature is sparse in comparing durability of alignment between non-operative and operative ASD patients.

Materials/Methods: ASD patients(scoliosis $\geq 20^{\circ}, \mathrm{SVA} \geq 5 \mathrm{~cm}, \mathrm{PT} \geq 25^{\circ}$, or $\left.\mathrm{TK} \geq 60^{\circ}\right)>18 \mathrm{yr}$ with baseline (BL)/1-year(1Y) radiographs. Operative(Op) and non-operative(N-Op) ASD patients were propensity score matched(PSM) for BL PT, PI-LL, SVA, age, Charlson comorbidity index(CCI), and frailty(ASD-FI). Kaplan-Meier analyses assessed durability of SVA, PI-LL, and PT alignment beginning at $1 Y$ postop for Op patients, and BL for N-Op. Alignment was durable if maintained within +1 standard deviation of age-adjusted ideal. Log Rank tests compared Op/N-Op survival distributions.

Results: 394 ASD patients(53 $\pm 17 \mathrm{yrs}, 83 \% \mathrm{~F})$ were included in the PSM analysis(197 Op, $197 \mathrm{~N}-\mathrm{Op})$. For Op patients, fusions spanned $11 \pm 4$ levels, and surgical approach included $71 \%$ posterior, $2 \%$ anterior, and $28 \%$ combined. No differences were observed in age, sex, or BMI between groups(all $\mathrm{P}>0.05$ ). Table 1 shows alignment and comorbidity differences between Op and N-Op groups at BL, $1 \mathrm{Y}$, and $2 \mathrm{Y}$ intervals. $\mathrm{N}-\mathrm{Op}$ patients showed less durable PI-LL alignment at $1 \mathrm{Y}$ (Non-Op: $63.5 \%$ vs Op: $73.6 \%)$ and $2 \mathrm{Y}(52.3 \%$ vs $65.3 \%, \mathrm{P}=0.026)$. Cumulative durability of $\mathrm{SVA}(\mathrm{P}=0.153)$ and $\mathrm{PT}(\mathrm{P}=0.708)$ did not differ between groups. Despite no BL differences, N-Op patients had higher rates of lung disease $(4.2 \%$ vs $0 \%, \mathrm{P}=0.016)$ and hypertension $(22 \%$ vs $10 \%, \mathrm{P}=0.006)$ at $2 \mathrm{Y}$ Despite no differences in $\mathrm{BL}$ frailty, a greater proportion of $\mathrm{N}-\mathrm{Op}$ patients were considered frail(ASD-FI $>3)$ at $1 \mathrm{Y}(24 \%$ vs $12 \%, \mathrm{P}=0.003)$ and $2 \mathrm{Y}(25 \%$ vs $13 \%, \mathrm{P}=0.007)$. Non-Op patients also showed inferior $\mathrm{BL}-2 \mathrm{Y}$ changes in frailty score $(1.2$ vs $-0.4, \mathrm{P}=0.001)$.

Discussion/Conclusion: Unlike operative, non-operative ASD patients showed progressive decline in spinopelvic alignment and inferior clinical outcomes over a 2-year period. As compared to operative, nonoperative patients also developed higher rates of pulmonary comorbidities and hypertension. 


\section{Paper 23. Pelvic Incidence Affects Age-Adjusted Alignment Outcomes in a Population of Adult Spinal Deformity}

Peter Passias, $M D^{l}$, Cole Bortz, $B A^{l}$, Frank Segreto, $B S^{l}$, Samantha Horn, $B A^{l}$, Katherine Pierce, $B A^{l}$, Haddy Alas, $B A^{2}$, Avery Brown, $B S^{l}$, Aaron Buckland, $M D^{3}$; Michael Gerling, $M D^{4}$

${ }^{1}$, New York, NY, ${ }^{2}$ SUNY Downstate College of Medicine, Brooklyn, NY, ${ }^{3}$ Department of Orthopedic Surgery, NYU Langone Orthopedic Hospital, NYU Langone Health, Manhattan, NY, ${ }^{4}$ NYU LMC School of Medicine, TriBeCa, NY

Background/Introduction: Current planning for surgical adult spinal deformity(ASD) patients takes into account patient age when establishing postoperative alignment targets for sagittal vertical axis(SVA), pelvic tilt(PT), and spino-pelvic-mismatch(PI-LL). Patient height and pelvic incidence(PI) have not been evaluated for their individual effects on achieving age-adjusted alignment targets.

Materials/Methods: Surgical ASD patients $>18 \mathrm{yr}$ with full-body $\mathrm{x}$-ray imaging at baseline(BL) and $<6 \mathrm{M}$ follow-up. Patients were grouped by percentile(low:<25th; normative:25th-75th; high: $>75$ th) for height and PI. Correction groups were generated at postoperative follow-up for actual alignment compared to ageadjusted ideal values for PT, PI-LL, and SVA, and PI-adjusted ideal alignment values for SS, as derived from clinically relevant formulas. Means comparison tests assessed differences in demographics and rates of matching ideal alignment $\left( \pm 10\right.$ year threshold for age-adjusted targets; $-7^{\circ}$ to $5^{\circ}$ measured minus ideal for SS) across height and PI groups.

Results: Included:205 ASD patients(59 $\pm 16 \mathrm{yr}, 64 \%$ F). Patients showed significant postoperative improvement in PT, PI-LL and SVA(all p<0.001). Breakdown of patient PI groups: low(25\%, $\left.38.5^{\circ} \pm 10.8^{\circ}\right)$, normative $\left(50 \%, 56.6^{\circ} \pm 5.4^{\circ}\right)$, high $\left(25 \%, 75.4^{\circ} \pm 7.1^{\circ}\right)$. Breakdown of height groups: low $(25 \%$, $1.51 \mathrm{~m} \pm 0.04 \mathrm{~m})$, normative $(50 \% 1.64 \mathrm{~m} \pm 0.05 \mathrm{~m})$, and high $(25 \%, 1.79 \mathrm{~m} \pm 0.06 \mathrm{~m})$. Age, BMI, sex did not differ across PI groups(all $\mathrm{p}>0.05$ ). Height groups did not differ in age or BMI(all $\mathrm{p}>0.05)$. Overall, $30 \%$ of patients met postoperative age-adjusted alignment target for PT, 24\% for PI-LL, and 29\% for SVA. 29\% of patients met PI-adjusted SS alignment. There were no differences across height groups in rates of achieving age-adjusted alignment ideals for PT, PI-LL, SVA, or SS (all $\mathrm{p}>0.05)$. Although there were no differences across PI groups in rates of achieving ideal alignment for PI-LL, SVA, or SS(all p>0 .05), patients with high PI reached ideal alignment for PT at a lower rate (15\%) than patients with normative(36\%) or low $\mathrm{PI}(33 \%, \mathrm{p}=0.031)$. Of the $58 \%$ of patients that matched any age-adjusted alignment target in early followup, there were no differences in postoperative HRQL outcomes across patient height or PI groups(all $\mathrm{p}>0.05$ ).

Discussion/Conclusion: Patients with high PI reached ideal postoperative PT alignment at a lower rate than patients with normative and low PI. Patient height had no impact on postoperative age-adjusted alignment outcomes. Current postoperative ideal alignment targets may warrant adjustment to account for the morphological factor of PI. 
Paper 24. Preoperative PHQ-2 Scoring Predicts Patient Satisfaction and Return to Work up to 1Year After Lumbar Fusion: A 2-Year Analysis from the Michigan Spine Surgery Improvement Collaborative (MSSIC)

Victor Chang, $M D^{l}$, Hesham Zakaria, $M D^{l}$, Michael Bazydlo, $M D^{l}$, Lonni Schultz, $M D^{l}$, Jad Khalil, MD ${ }^{2}$; Brad Webb, DO ${ }^{3}$

${ }^{1}$, Detroit, MI, ${ }^{2}$ Michigan Orthopaedic Institute ,Southfield, MI, ${ }^{3}$ Beaumont Hospital and Medical Center, Royal Oak, MI

Background/Introduction: The Michigan Spine Surgery Improvement Collaborative (MSSIC) is a prospective, longitudinal, multicenter, quality improvement collaborative.1 Using MSSIC, we sought to identify the relationship between a positive PHQ-2, which is predictive of depression, and patient satisfaction, return to work, and achieving ODI MCID up to 2-years after lumbar fusion.

Materials/Methods: A total of 8585 lumbar fusion patients were analyzed. Patient satisfaction was measured by the NASS patient satisfaction index. A positive PHQ-2 score is $=3$, which has a $82.9 \%$ sensitivity and $90.0 \%$ specificity in detecting major depressive disorder.2 Multivariate logistic regression models were constructed; variables tested include age, gender, race, $\mathrm{PMH}$, preoperative diagnosis (disc herniation, spondylolisthesis, etc), preoperative symptoms (axial pain, radicular pain, etc), severity of surgery, and preoperative opioid usage.

Results: Multivariate analysis was performed. Patients with a positive PHQ- 2 score were less likely to be satisfied after lumbar fusion at 90- days (OR 0.93, p<0.001), 1-year (OR 0.92, p=0.001), and 2-years (OR $0.92, \mathrm{p}=0.028$ ). A positive PHQ-2 was also associated with decreased likelihood of returning to work at 90 days (OR 0.76, p<0.001), 1-year (OR 0.85, p=0.001), and at 2-years (OR 0.82, p=0.031). A positive PHQ-2 was predictive of failure to achieve ODI MCID at 90-days (OR 1.07, p=0.005) but not at 1-year or 2-years after lumbar fusion.

Discussion/Conclusion: A multivariate analysis from a large, multicenter, prospective database on lumbar fusion patients was performed. We find that PHQ-2, which is a simple and accurate screening tool for depression, predicts and inability to return to work and worse satisfaction up to 2-years after lumbar fusion. Depression is a treatable condition, and so in the same way that patients are medically optimized before surgery to decrease postoperative morbidity, then perhaps patients should have preoperative psychiatric optimization to improve postoperative functional outcomes. 


\section{Paper 25. Does Obesity Impact Clinical Outcomes and Lumbar Sagittal Alignment after a Posterior Lumbar Spine Fusion?}

Jannat Khan, $B S^{l}$; Bryce Basques, $M D^{2}$, Kyle Kunze, $B S^{l}$, Gagan Grewal, $B S^{l}$, Young Soo Hong, $B S^{l}$, Coralie Pardo, $B S^{1}$, Philip Louie, $M D^{2}$, Matthew Colman, $M D^{3}$, Howard An, $M D^{2}$

${ }^{1}$, Chicago, IL, ${ }^{2}$ Rush University Medical Center, Chicago, IL, ${ }^{3}$ Rush University, Chicago, IL

Background/Introduction: Obesity is an increasingly common comorbidity in patients undergoing posterior lumbar spinal fusion (PLF). However, information about the effect of obesity on radiographic and clinical outcomes following PLF is less well understood. Therefore, this study compared obese and nonobese patients undergoing PLF.

Materials/Methods: A retrospective cohort study was conducted of consecutive patients who underwent primary elective open PLF. Patients were excluded from analysis if they were under 18 years of age at the time of surgery or had a fusion to treat a lumbar fracture, tumor, or infection. Patients were classified as obese per Center for Disease Control and Prevention guidelines if their body mass index (BMI) was above $30 \mathrm{~kg} / \mathrm{m} 2$. Other patient and operative characteristics were collected and compared between obese and nonobese groups. Preoperative and final postoperative Visual Analog Scale (VAS) Back/Leg pain, and Oswestry Disability Index (ODI), were collected. Preoperative, immediate postoperative, and final radiographs were assessed to measure lumbar lordosis (LL), pelvic tilt (PT), pelvic incidence (PI), and the PI-LL difference was calculated. Additionally, postoperative complications, pseudoarthrosis, and achievement of minimally clinically important difference for VAS back, VAS leg, and ODI were collected.

Results: A total of 974 patients were included; 506 (51.95\%) patients with BMI $<30$ (non-obese) and 468 $(48.05 \%)$ patients with $\mathrm{BMI} \geq 30$ (obese). Obese patients were more likely to have diabetes mellitus $(\mathrm{p}<0.001)$ and American Society of Anesthesiologists $\geq 3(\mathrm{p}<0.001)$. On multivariate linear regression, obese patients had significantly longer operative times $(\mathrm{p}<0.001)$ and postoperative length of stay $(\mathrm{p}<0.001)$ compared to non-obese patients. There was no difference in radiographic measurements, patientreported outcomes, postoperative complications, or reoperations between groups.

Discussion/Conclusion: Obese patients had significantly more comorbidities, longer operative time, extended postoperative length of stay compared to non-obese patients. However, sagittal parameters, patient-reported outcomes, inpatient complications, and reoperations were similar between the two groups. Given these findings, open PLF can be considered safe and effective in obese patients after thorough consideration of related comorbidities. 
Paper 26. Ambulation on POD\#0 is Associated with Decreased Adverse Events After Elective Lumbar Spine Surgery: Analysis of the Michigan Spine Surgery Improvement Collaborative (MSSIC)

DANIEL POSSLEY, DO, MS ${ }^{l}$, Jad Khalil, MD ${ }^{2}$, Hesham Zakaria, $M D^{3}$, KARAM ASMARO, MD ${ }^{4}$, Richard Rammo, $\mathrm{MD}^{4}$, THOMAS NOH, MD ${ }^{4}$, Michael Bazydlo, $\mathrm{MD}^{3}$, Lonni Schultz, $M D^{3}$, Victor Chang, $M D^{3}$, Joseph Schwab, $M D, M S^{5}$

'CORNERSTONE ORTHOPAEDICS AND SPORTS MEDICINE, WHEAT RIDGE, CO ${ }^{2}$ Michigan Orthopaedic Institute , Southfield , MI,${ }^{3}$, Detroit, MI ${ }^{4}$, DETROIT, MI, ${ }^{5}$ MGH, Boston, MA

Background/Introduction: The Michigan Spine Surgery Improvement Collaborative (MSSIC) is a multicenter quality improvement collaborative.1 Using the MSSIC database, we sought to identify the relationship between ambulation on the day of surgery (POD\#0) and 90-day adverse events after lumbar surgery, specifically urinary retention (UR), urinary tract infection (UTI), ileus, readmission, surgical site infection (SSI), PE/DVT, and disposition to a rehab facility.

Materials/Methods: A total of 23295 lumbar surgery patients were analyzed. Multivariate logistic regression models were constructed, including variables on patient demographics, medical history, and surgical intensity. Matching was performed to account for unknown confounding variables.

Results: POD\#0 ambulation was associated with decreased LOS (OR0.83, $\mathrm{p}<0.001)$, UR (OR0.73, $\mathrm{p}=0.008)$, UTI (OR0.52, $\mathrm{p}=0.001)$, ileus (OR0.52, $\mathrm{p}<0.001), 30$-day $(\mathrm{OR} 0.84, \mathrm{p}=0.035)$ and 90 -day (OR0.86, $\mathrm{p}=0.009)$ readmission, and rehab discharge (OR0.52, $\mathrm{p}<0.001)$. POD\#0 ambulation after singlelevel decompression (6244 patients) decreased LOS (OR0.72, $\mathrm{p}<0.001)$, UR (OR0.73, $\mathrm{p}=0.004)$, UTI (OR0.43, $\mathrm{p}=0.003)$, and rehab discharge (OR0.18, $\mathrm{p}<0.001)$. Ambulation after multi-level decompression (5526 patients) was associated with decreased LOS (OR0.73, $\mathrm{p}<0.001)$, UR (OR0.75, $\mathrm{p}=0.04)$, ileus (OR0.60, $\mathrm{p}=0.027)$, and rehab discharge (OR0.44, $\mathrm{p}<0.001)$. Ambulation after single-level fusion $(5790$ patients) decreased LOS (OR0.85 p<0.001), 30-day readmission (OR0.77, $\mathrm{p}=0.032)$ and rehab discharge (OR0.65, $\mathrm{p}=0.004)$. Ambulation after multi-level fusion (5735 patients) decreased LOS (OR0 .88, $\mathrm{p}<0.001$ ), UTI (OR0.60, p=0.003), ileus (OR0.51, p=0.02), 30-day readmission (OR0.77, $\mathrm{p}=0.032$ ), and rehab discharge $(\mathrm{OR} 0.59, \mathrm{p}<0.001)$. No change in rate of or DVT/PE was observed for patients who ambulated POD\#0.

Discussion/Conclusion: POD\#0 ambulation is associated with a significantly decreased risk for several key adverse events after lumbar spine surgery. Decreasing the incidence of these outcomes would be associated with significant cost savings. As ambulation POD\#0 is a modifiable factor in any patient's postoperative care following most spine surgery, it should be encouraged and incorporated into spine related enhanced recovery after surgery (ERAS) programs. 
Paper 27. Pre-operative muscle health impacts the time it takes to reach minimally clinically important differences in health related quality of life scores for one level lumbar fusions

Sohrab Virk, $M D^{1}$, Jung Mok, $B S^{2}$, Avani Vaishnav, $M B B S^{3}$, Steven McAnany, $M D^{3}$, Sravisht Iyer, $M D^{4}$, Todd Albert, $M D^{4}$, Catherine Himo Gang, $M P H^{5}$, Sheeraz Qureshi, $M D, M B A^{4}$

${ }^{1}$, New York, New York, ${ }^{2}$ Weill Cornell Medical College, New York, NY, ${ }^{3}$ Hospital for Special Surgery, New York, New York, ${ }^{4}$ Hospital for Special Surgery, New York, NY, ${ }^{5}$, New York, NY

Background/Introduction: There is evidence that degenerative changes in paralumbar musculature impact conditions like low back pain and spinal stenosis. We hypothesized that paralumbar muscle health would alter the time it took to reach minimal clinically important differences (MCIDs) in health-related quality of life scores (HRQOLs) for patients undergoing lumbar surgery.

Materials/Methods: We performed a retrospective review of patients that had a lumbar decompressive surgery or a one level lumbar spinal fusion. We analyzed MRI to quantify muscle health using the lumbar indentation value (LIV) which is a validated method of measuring the cross-sectional area of lumbar musculature. HRQOL scores were collected on these patients in the pre-operative period and the postoperative period up to 1 year out from surgery. These scores included the VAS leg, VAS back, ODI, SF-12 mental health scores (MHS) and SF-12 physical health scores (PHS). We correlated the LIV calculated off pre-operative MRI and correlated this finding with time to MCID using a linear regression analysis.

Results: Eighty-five patients were included. The average age was 58.4+/-15.7 and there were 45 men and 40 women. The most common diagnosis for lumbar decompression (LD) and lumbar fusion (LF) was disc herniation (49.2\%) and lumbar spinal stenosis (93.1\%), respectively. The average LIV for LD and LF patients was $16.2+/-6.5 \mathrm{~mm}$ and $17.1+/-6.6 \mathrm{~mm}$, respectively. There was no statistically significant correlation between time to MCID for HRQOL scores and LIV for LD patients. For LF patients, there was a statistically significant inverse relationship between time to MCID for ODI $(p=0.02)$ and LIV and time to MCID for SF-12 MHS ( $\mathrm{p}=0.04$ ) and LIV (i.e higher LIV correlated with lower time to MCID for ODI and SF-12 MHS).

Discussion/Conclusion: These results emphasize the need for evaluation of paralumbar muscle health for patients undergoing lumbar surgery. Specifically, lumbar muscle health correlates with time to MCID for ODI and SF-12 MHS for patients undergoing lumbar fusion surgery. Further investigations are needed to determine how improvement of cross-sectional area of paralumbar muscle impacts MCID for patients undergoing lumbar surgery. 


\section{Paper 28. BMI Does Not Affect Short-Term Patient Reported Outcomes after Lumbar Decompression Surgery}

Dhruv Goyal, BA ${ }^{l}$; Srikanth Divi, M.D. ${ }^{l}$, Matthew Galetta, $B A^{l}$, Kristen Nicholson, PhD ${ }^{l}$, Jeff Rihn, $M D^{2}$, Mark Kurd, $M D^{I}$, Alan Hilibrand, $M D^{2}$, Chris Kepler, $M D M B A^{3}$, Alexander Vaccaro, $M D P h D M B A^{4}$, Gregory Schroeder, M.D. ${ }^{2}$

${ }^{1}$, Philadelphia, PA, ${ }^{2}$ Rothman Institute, Thomas Jefferson University Hospital, Philadelphia, PA, ${ }^{3}$, Philadelphia ,PA, ${ }^{4}$ Rothman Institute, Philadelphia, PA

Background/Introduction: While it is well known that an increased preoperative body mass index (BMI) increases the risk of complications following surgery, there is a lack of evidence regarding its influence on patient reported outcome measurements (PROMs) following lumbar decompression surgery. The goal of this study was to elucidate how BMI affects PROMs in patients undergoing decompression surgery for degenerative disease.

Materials/Methods: Patients undergoing lumbar decompression surgery between one to three levels at a single center, academic hospital were retrospectively identified. Patients under the age of 18 years, and those undergoing surgery for infection, trauma, tumor, or revision, and less than one year follow-up were excluded. Patients were split into groups based on preoperative BMI: Group 1) BMI < 25.0; Group 2) BMI 25.0-29.9; Group 3) BMI 30.0-34.9; and Group 4) BMI > 35.0. Absolute PROM scores, the recovery ratio and the percent of patients achieving MCID between groups were compared and a multiple linear regression analysis was performed.

Results: A total of 195 patients were included (Table 1). Thirty-four (17.4\%) patients were in Group 1, 83 $(42.6 \%)$ were in Group 2, $47(24.1 \%)$ were in Group 3, and 31 (15.9\%) were in Group 4. There were no significant differences between BMI groups at baseline or postoperatively for each outcome measure. All groups improved from baseline as noted by the recovery ratio, except for MCS-12 scores in Group 3 and Group 4, however this trend did not reach significance $(\mathrm{p}=.080)$. The percent of patients reaching MCID was not significantly different between groups for any outcome measure. Multiple linear regression analysis revealed that BMI was not a significant predictor for change in outcomes for any measure. The 30day readmission rate was $1.03 \%, 90$-day readmission rate was $3.59 \%$, and overall revision rate at the 2-year follow-up was $3.1 \%$, with no significant differences between groups ( $\mathrm{p}>.05)$.

Discussion/Conclusion: While BMI is known increase complication rates in surgery, our results suggest that BMI may not significantly affect short-term patient outcomes in patients undergoing lumbar decompression surgery. More prospective evidence is needed to explore this relationship. 


\section{Paper 29. Preoperative Depression Affects Health-Care Related Quality of Life Following Lumbar Fusion}

Srikanth Divi, M.D. ${ }^{l}$, Dhruv Goyal, BA ${ }^{l}$, Matthew Galetta, BA ${ }^{l}$, Kristen Nicholson, PhD ${ }^{l}$, Kris Radcliff, $M D^{2}$, Barrett Woods, $M D^{3}$, Alan Hilibrand, $M D^{4}$, Chris Kepler, $M D M B A^{5}$, Alexander Vaccaro, $M D P h D$ $M B A^{6}$, Gregory Schroeder, M.D. ${ }^{4}$

${ }^{1}$, Philadelphia, PA,${ }^{2}$ Rothman, Philadelphia, PA, ${ }^{3}$ Rothan Institute, Philadelphia, Pennsylvania, ${ }^{4}$ Rothman Institute, Thomas Jefferson University Hospital, Philadelphia, PA, ${ }^{5}$, Philadelphia ,PA, ${ }^{6}$ Rothman Institute, Philadelphia, PA

Background/Introduction: The impact of preexisting depression on health-care related quality of life (HRQOL) outcomes following lumbar spine surgery is not well described. In this study, we identified depression using two criteria and used it to correlate with patient outcomes.

Materials/Methods: Patients undergoing lumbar fusion between one to three levels at a single center, academic hospital were retrospectively identified. Patients under the age of 18 years, and those undergoing surgery for infection, trauma, tumor, or revision, and less than one year follow-up were excluded. Patients with depression were identified using an existing clinical diagnosis or a score of MCS-12 $\leq 45.6$ on the preoperative SF-12 survey. Absolute HRQOL scores, the recovery ratio and the percent of patients achieving MCID between groups were compared, and a multiple linear regression analysis was performed.

Results: 383 patients were included in the total cohort, with 126 (32.9\%) patients reporting symptoms of depression based on MCS-12 and 257 (67.1\%) without depression. Using a preexisting clinical diagnosis, only $25(7.0 \%)$ patients were identified with depression. Using the MCS-12 threshold, preoperative scores except for PCS-12, and all postoperative scores were significantly different $(\mathrm{p}<.05)$ with the depression group reporting higher disability and pain in all domains. Using a preexisting diagnosis of depression, only preoperative ODI scores were significantly different ( $\mathrm{p}=.009)$, with worse scores in the depression group. There was a trend towards a lower proportion of patients achieving MCID for PCS-12 scores in patients with depression identified by MCS-12 ( $\mathrm{p}=.087)$. Recovery ratio analysis and multiple regression analysis indicated patients with depression identified by MCS-12 improved significantly less in all outcome measures $(\mathrm{p}<.05)$. These differences disappeared when stratifying patients based on preexisting diagnosis of depression.

Discussion/Conclusion: While patients in all groups improved across all outcome measures after surgery, those with symptoms of depression identified by preoperative MCS-12 score showed a lower magnitude of improvement. This difference was not seen when patients were stratified by preexisting diagnosis of depression. The findings in this study emphasize that symptoms of depression may exist sub-clinically in many patients undergoing surgery and can affect patient outcomes. 
Paper 30. Chronicity of Preoperative Opioid Usage Predicts Patient Satisfaction, Return to Work, and Achieving ODI MCID up to 2 Years After Lumbar Fusion: Analysis from the Michigan Spine Surgery Improvement Collaborative (MSSIC)

Hesham Zakaria, $M D^{I}$, Victor Chang, $M D^{l}$, Michael Bazydlo, $M D^{l}$, Lonni Schultz, MD ${ }^{l}$, Jad Khalil, MD ${ }^{2}$; Yi Guo, $M D^{3}$

${ }^{1}$, Detroit, MI, ${ }^{2}$ Michigan Orthopaedic Institute , Southfield , MI, ${ }^{3}$, Royal Oak, MI

Background/Introduction: The Michigan Spine Surgery Improvement Collaborative (MSSIC) is a multicenter quality improvement collaborative. Using the MSSIC database, we sought to identify the relationship between preoperative opioid usage and patient satisfaction, return to work, and achieving ODI MCID up to 2-years after lumbar fusion.

Materials/Methods: A total of 8693 lumbar fusion patients were analyzed. Patient satisfaction was measured by the NASS patient satisfaction index. Multivariate logistic regression models were constructed; variables tested include age, gender, race, $\mathrm{PMH}$, and number of surgical levels. Preoperative opioid chronicity was defined as opioid naïve (no opioid usage), new opioid users $(<6 \mathrm{wks})$, recent opioid users $(6 \mathrm{wks}-3 \mathrm{mo})$, frequent opioid users $(3 \mathrm{mo}-6 \mathrm{mo})$, and chronic opioid users $(>6 \mathrm{mo})$.

Results: Multivariate analysis was performed. Comparisons were done to opioid naïve patients. Chronic opioid users were less likely to be satisfied with their procedure at 90-days (OR 0.95, p<0.001), 1-year (OR $0.90, \mathrm{p}<0.001)$, and 2-years (OR $0.87, \mathrm{p}<0.001)$ after surgery. New opioid users were more likely to achieve ODI MCID at 90-days (OR 1.25, p<0.001), 1-year (OR 1.15, p<0.001), and 2-years (OR 1.22, $\mathrm{p}<0.002)$ postoperatively. Recent opioid users were more likely to achieve ODI MCID at 2 years (OR 1.26, $\mathrm{p}<0.001)$. Chronic opioid users were less likely to achieve ODI MCID at 90-days (OR 0.89, $\mathrm{p}<0.001), 1$ year (OR 0.87, p=0.002), and 2-years (OR 0.82, p=0.004). Chronic opioid users were also less likely to return to work at 90-days (OR $0.83, \mathrm{p}<0.001)$.

Discussion/Conclusion: A multivariate analysis on preoperative opioid usage from a large, multicenter, prospective database on lumbar fusion patients was performed. We found that, as compared to opioid naïve patients, 2-years postoperatively new opioid users were more likely to have a favorable outcome, while chronic opioid users were less likely to have a favorable outcome. Thus, preoperative opioid counseling is imperative to improve outcomes in spine surgery. 


\section{Paper 31. Durability and outcomes of minimally-invasive TLIF and adjacent laminectomy for grade I spondylolisthesis}

Anan Shtewe, $M D^{I}$, Bledi Brahimaj, $M D^{2}$, John O'Toole, $M D, M S^{3}$, Harel Deutsch, $M D^{2}$, Richard Fessler, $M D, P h D^{3}$, Ricardo Fontes, $M D^{l}$

${ }^{I}$ Rush University Medical Center, chicago, IL,${ }^{2}$, Chicago, IL,${ }^{3}$ Rush University Medical Center, Chicago, IL

Background/Introduction: To determine the re-operation rate and outcomes of patients undergoing simultaneous one-level MIS TLIF and adjacent-level laminectomy for grade one spondylolisthesis and adjacent stenosis.

Materials/Methods: Retrospective analysis of prospectively collected data at a single institution (20072017) of all patients who undergoing simultaneous MIS TLIF and laminectomy for stenosis adjacent to spondylolisthesis requiring short segment fusion. The primary outcome was reoperation rate and secondary outcomes were length of hospital stay (LOS), estimated blood loss (EBL), ODI and SF-12 scores.

Results: 37 patients were included (54\% male and $46 \%$ female) with average age of 67.5 years. Operative time was 244 minutes with 43 levels fused and 61 laminectomies. Estimated blood loss was $235 \mathrm{ml}$. Length of hospital stay was 5 days. The incidence of back pain was $92 \%, 19 \%$ had intermittent neurologic claudication and $86 \%$ had radicular symptoms. Seventy percent were non-smokers. The overall reoperation rate was $13.5 \%$ with a follow up average of 22 months. Two patients had recurrent stenosis at the level above the fusion. Two patients were re-operated due to pseudoarthrosis and one patient developed new symptoms a month after surgery $\mathrm{d} / \mathrm{t}$ a fall and required decompression. The average ODI was 35.7 while SF-12 was 34.5 .

Discussion/Conclusion: The addition of decompression alone secondary to stenosis at the adjacent level of short segment fusion during the same operation in patients with grade one spondylolisthesis has a $13.5 \%$ incidence of re-operation of all causes, where re-operation due to adjacent segment disease (ASD) was $5.4 \%$ with a mean follow up of 22 months. Recently published data report a higher incidence of reoperation rate for ASD to be more than $10 \%$. These findings show that this procedure is durable with acceptable re-operation rate and good outcomes. A prospective study to further determine the ability of such procedure to reduce the risk for symptomatic ASD is needed. 


\section{Paper 32. Greater PHQ-9 Score Predicts Worse Clinical Outcomes Following Minimally Invasive Transforaminal Lumbar Interbody Fusion}

Dil Patel, $B S^{l}$; Joon Yoo, $B A^{l}$, Benjamin Khechen, $B A^{2}$, Brittany Haws, $B S^{2}$, Sailee Karmarkar, $B S^{l}$, Eric Lamoutte, $B S^{l}$, Kern Singh, $M D^{3}$

${ }^{l}$, Chicago, IL,${ }^{2}$ Rush University Medical Center, Chicago, Illinois, ${ }^{3}$ Rush University Medical Center, Chicago, IL

Background/Introduction: Few studies have evaluated the relationship between depression and patientreported outcomes (PROs), postoperative pain, and narcotics utilization following minimally invasive spinal surgery. This study aims to determine if there exists an association between preoperative depression, assessed by Patient Health Questionnaire-9 (PHQ-9), and PROs, inpatient pain, and narcotics utilization after minimally invasive transforaminal lumbar interbody fusion (MIS TLIF).

Materials/Methods: Patients undergoing primary, single-level MIS TLIF were retrospectively reviewed and stratified by preoperative PHQ-9 scores: no depression ( $<5)$, mild depression (5-9), and moderate-tosevere depression $(\geq 10)$. PROs including Oswestry Disability Index (ODI), Veterans RAND (VR)-12 Mental Component Score (MCS) and Physical Component Score (PCS), and Visual Analog Scale (VAS) back and leg pain scores were collected preoperatively and at 6-week, 12-week, and 6-month follow-up. Inpatient pain scores were expressed using the Visual Analogue Scale (VAS), and narcotics utilization was calculated using Oral Morphine Equivalents (OMEs). Multivariate linear regression and chi-squared analysis were used to determine if an association existed between PHQ-9 score groups and baseline and perioperative characteristics. Multivariate linear regression was utilized to assess for an association between PHQ-9 and postoperative PROs.

Results: 94 patients were included. 47 had a PHQ-9 score $<5,22$ had a PHQ-9 score of 5-9, and 24 had a PHQ $\geq 10$. Patients with higher PHQ-9 scores were younger, more obese, and carried Workers' Compensation insurance. Higher PHQ-9 scores were also associated with greater inpatient VAS pain scores on postoperative day 0 and 1 and demonstrated greater hourly narcotics consumption on postoperative day 0 . Higher PHQ-9 scores were associated with worse preoperative ODI, VR-12 MCS and PCS, and VAS back and leg pain scores and worse postoperative scores in all PROs at each postoperative time point.

Discussion/Conclusion: The results of this study suggest depression symptoms are associated with increased preoperative disability, pain, and narcotic utilization. Additionally, patients with greater PHQ-9 demonstrate less clinical improvement postoperatively compared to patients with a PHQ- $9<5$ after MIS TLIF. As such, patients with greater preoperative PHQ-9 scores should be counseled regarding postoperative outcomes to better manage expectations and satisfaction. 


\section{Paper 33. Return to Activities and Discontinuation of Narcotics after Minimally Invasive Spine Surgery}

Jung Mok, $B S^{l}$, Avani Vaishnav, $M B B S^{2}$, Steven McAnany, $M D^{2}$, Todd Albert, $M D^{3}$, Sravisht Iyer, $M D^{3}$, Catherine Himo Gang, $\mathrm{MPH}^{4}$, Sheeraz Qureshi, $M D, M B A^{3}$

${ }^{l}$ Weill Cornell Medical College, New York, NY, ${ }^{2}$ Hospital for Special Surgery, New York, New York, ${ }^{3}$ Hospital for Special Surgery, New York, NY, ${ }^{4}$, New York, NY

Background/Introduction: This study compares the time to return to activities, namely return to work and return to driving, and time to discontinuation of narcotics, between MI single-level lumbar decompression and MI single-level lumbar fusion.

Materials/Methods: This is a retrospective, single academic center, single surgeon cohort study. To be included, patients had to have been driving or working pre-operatively, or had to have been administered narcotics post-operatively. The number of days it took for patients to return to the activity or to discontinue narcotics was calculated. Independent samples median t-tests and independent samples Mann-Whitney Utests were conducted to assess for differences between the two procedures. The percentage of patients who returned to activities/discontinued narcotics at various time-points $(15,30,60,90,120$, and 150 days) were also calculated, and chi2-tests were conducted to compare those percentages between the two surgeries.

Results: In return to driving analysis, 36 patients underwent MI single-level decompression (median days to return to driving $=14$ days), and 41 patients underwent MI single-level lumbar fusion (median $=16$ days). In return to work analysis, 38 patients underwent decompression (median days to return to work $=$ 16.5 days), and 31 patients underwent fusion (median $=14$ days). In discontinuation of narcotics analysis, 41 patients underwent decompression (median days to discontinue narcotics $=7$ days), and 44 patients underwent MI fusion (median = 11.5 days). For all three measures, there were no significant differences in demographic variables between those who underwent decompression and those who underwent fusion procedures. There were no statistically significant differences in the median number of days to return to driving, return to work, or discontinuation of narcotics, or in the distributions of those values.

Discussion/Conclusion: Return to activities and discontinuing narcotics after spine surgery are important aspects of patient expectations for recovery. It is well known that open lumbar fusion patients take significantly longer to return to activities and discontinue narcotics compared to open lumbar decompression patients. This study shows that utilizing minimally invasive methods for fusion may allow fusion patients to recover faster, and hence potentially eliminate the difference seen in open techniques. 
Paper 34. Ultrasound Measurement of the Skin as a Potential Marker of Bone Quality: A Prospective Pilot Study of Patients undergoing Lumbar Spinal Fusion

Stephan Salzmann, $M D^{l}$, Ichiro Okano, $M D^{l}$, Colleen Rentenberger, $M D^{2}$, Courtney Ortiz Miller, $B A^{3}$, Paul Schadler, $M D^{2}$, Theodore Miller, $M D^{2}$, Andrew Sama, $M D^{I}$, Frank Cammisa, $M D^{l}$, Federico Girardi, $M D^{4}$, Alexander Hughes, $M D^{l}$

${ }^{l}$ Hospital for Special Surgery, New York, New York, ${ }^{2}$, New York, New York, ${ }^{3}$ Hospital for Special Surgery, New York, New York, ${ }^{4}$, New york, New York

Background/Introduction: Bone mineral density (BMD) is not the sole predictor of fracture development. Qualitative markers including bone collagen maturity contribute to bone fragility. Clinically, bone quality assessment with Fourier-transform infrared spectroscopy (FTIR) is limited since a bone biopsy is required. Bone and related type I collagen containing connective tissues degenerate in parallel fashion. Using ultrasound (US), a decrease in dermal thickness and an increase in reticular layer echogenicity has been observed with aging due to an accumulation of degenerated collagen. We hypothesized that US dermal thickness and echogenicity correlate with bone collagen maturity.

Materials/Methods: 60 patients undergoing instrumented, posterior lumbar fusion were prospectively enrolled. Spine quantitative computed tomography (QCT) and skin US measurements were performed. Dermal layer thickness and echogenicity of the reticular dermis were calculated (Figure 1). During surgery, bone biopsies (iliac crest and pedicle) were obtained. Collagen maturity (ratio of mature to immature collagen cross-links) was assessed with FTIR. Pearson's correlation and linear regression tests were performed in Caucasian patients without anti-osteoporotic medications.

Results: 43 patients (mean age 61 years, 24 females) met inclusion criteria. Among men, there was no correlation between US measurements and collagen maturity. Among women, US dermal layer thickness correlated negatively with collagen maturity in trabecular bone of the iliac crest $(\mathrm{r}=-0.51, \mathrm{p}=0.01)$ and vertebra $(\mathrm{r}=-0.59, \mathrm{p}=0.01)$ as well as in cortical bone of the iliac crest $(\mathrm{r}=-0.50, \mathrm{p}=0.02)$ and vertebra $(\mathrm{r}=-$ $0.50, \mathrm{p}=0.04)$. In addition, echogenicity correlated positively with collagen maturity in trabecular vertebral bone $(r=0.59, p=0.01)$. After age adjustment, US measurements and collagen maturity remained significant. In both genders, US measurements showed no correlation with QCT BMD measurements.

Discussion/Conclusion: This study is the first to elucidate the relationship of collagen quality in skin and bone. Previous studies showed that skin thickness is only a poor predictor of BMD but demonstrated the ability to discriminate fracture patients from controls. This discrepancy could possibly be explained by our results, which suggest a significant correlation of skin US measurements with bone quality (collagen maturity) rather than bone quantity (BMD). In addition, our study suggests obvious gender differences. In summary, similar factors may determine both dermal layer and bone quality. 
Paper 35. Two-Year Outcomes of Patients Treated with BVN Ablation for the Relief of Chronic Low Back Pain: Results of the SMART Trial

\author{
Alfred Rhyne, $M D^{1}$, Jeffrey Fischgrund, $M D^{2}$, Rick Sasso, $M D^{3}$, Hyun Bae, $M D^{4}$ \\ ${ }^{1}$ OrthoCarolina, Charlotte, North Carolina, ${ }^{2}$, Royal Oak, Michigan, ${ }^{3}$, Carmel, IN,${ }^{4}$ Cedars-Sinai Spine \\ Center, Los Angeles, $\mathrm{CA}$
}

Background/Introduction: Thirty percent of Americans have low back pain (LBP) at any given time, leading to approximately 50 million physician visits in the U.S. annually. Chronic low back pain (CLBP) can be difficult to diagnose and treat using either non-surgical therapies or surgical interventions.

Antonacci et al. proposed that some pain previously ascribed to the disc actually emanates from the vertebral endplate nociceptors which communicate to the CNS through the basivertebral nerve (BVN).1 The purpose of the present study is to report the 2-year clinical outcomes for CLBP patients treated with radiofrequency $(\mathrm{RF})$ ablation of the $\mathrm{BVN}$ in a randomized controlled trial (RCT) that previously reported 1year follow-up.

Materials/Methods: A total of 147 patients were treated with RF Ablation of the BVN in an RCT designed to demonstrate safety and efficacy as part of a Food and Drug Administration-Investigational Device Exemption (FDA-IDE) trial. Evaluations, including patient self-assessments, physical and neurological examinations, and safety assessments, were performed at two and six weeks, and three, six, twelve, eighteen, and twenty-four months postoperatively.

Results: Clinical improvements in ODI, VAS, and SF-36 PCS were statistically significant compared to baseline at all follow-up time points through 2 years. The mean percent improvements in ODI and VAS compared to baseline at 2 years were $53.7 \%$ and $52.9 \%$, respectively. Responder rates for ODI and VAS were also maintained through 2 years with patients showing clinically meaningful improvements in both: ODI $\geq 10$-point improvement in $76.4 \%$ of patients and ODI $\geq 20$-point improvement in $57.5 \%$; VAS $\geq 1.5$ $\mathrm{cm}$ improvement in $70.2 \%$ of patients.

Discussion/Conclusion: Patients treated with RF ablation of the BVN for CLBP exhibited sustained clinical benefits in ODI and VAS and maintained high responder rates at 2 years following treatment. BVN ablation appears to be a durable, minimally invasive treatment for the relief of CLBP. 
Paper 36. A Comparative Study of Freshly Isolated Adipose-Derived and Bone Marrow-Derived Stem Cells in a Rat Posterolateral Spinal Fusion Model

Christina Holmes, $P h D^{1}$; Alexander Perdomo-Pantoja, $M D^{2}$, Maritza Taylor, $B A^{3}$, Colson Tomberlin, $B S^{4}$, Wataru Ishida, $M D^{5}$, Ethan Cottrill, $M S^{6}$, Sheng-Fu Lo, $M D^{l}$, Timothy Witham, $M D^{l}$

${ }^{1}$ Johns Hopkins University School of Medicine, Baltimore, MD, ${ }^{2}$ Johns Hopkins University School of

Medicine, Baltimore, Maryland, ${ }^{3}$, BALTIMORE, Maryland, ${ }^{4}$, Baltimore, Maryland, ${ }^{5}$ Johns Hopkins

University School of Medicine, BALTIMORE, Maryland, ${ }^{6}$ Johns Hopkins School of Medicine,

Baltimore, $M D$

Background/Introduction: Adipose-derived stem cells (ADSCs) offer clinical advantages in spinal fusion over bone marrow-derived stem cells (BMSCs), including larger available tissue volumes, higher stem cell concentrations, and reduced donor site morbidity. While pre-clinical studies have shown that ex vivo expanded ADSCs can be successfully used in spinal fusion, the use of freshly isolated cells will better allow clinical translation. We analyzed the efficacy of freshly isolated ADSCs and BMSCs in achieving successful spinal fusion combined with a clinical-grade bone graft substitute in a rat model.

Materials/Methods: ADSCs were isolated from the inguinal fat pads, while BMSCs were isolated from the long bones of syngeneic 6-8 week old Lewis rats and combined with Vitoss (Stryker) bone graft substitute for subsequent transplantation. Posterolateral spinal fusion surgery at L4-5 was performed on 27 female Lewis rats divided into 3 experimental groups: [1] Vitoss bone graft substitute only (VO group, $\mathrm{n}=9)$; [2] Vitoss $+2.5 \times 10^{\wedge} 6$ ADSCs/side $(\mathrm{n}=9)$; and, [3] Vitoss $+2.5 \times 10^{\wedge} 6 \mathrm{BMSCs} / \mathrm{side}(\mathrm{n}=9)$. Fusion was assessed eight weeks post-surgery. Manual palpation scoring evaluated the motion across the operated joint, and MicroCT images were assessed for fusion mass volume and CT fusion.

Results: MicroCT imaging analyses revealed that fusion volumes and CT fusion scores in the ADSC group were significantly higher than in the VO group $\left(22.07 \mathrm{~mm}^{\wedge} 3 \mathrm{vs} .14 .9 \mathrm{~mm}^{\wedge} 3\right.$, respectively, $\mathrm{p}=.006$, and 1.58 vs. 1.08, respectively, $\mathrm{p}=.007$ ); In the BMSC group, fusion volumes were significantly higher than in the VO group (19.54 $\mathrm{mm}^{\wedge} 3$ vs. $14.9 \mathrm{~mm}^{\wedge} 3$, respectively, $\left.\mathrm{p}=.03\right)$. CT volume and fusion score were not significantly different between the ADSC group and the BMSC group $\left(22.07 \mathrm{~mm}^{\wedge} 3\right.$ vs. $19.54 \mathrm{~mm}^{\wedge} 3$, and 1.58 vs. 1.33 , respectively. $P>.05$ ). Average manual palpation scores were not significantly different between groups (1.4 versus 1.2 versus 1.0 , respectively, $\mathrm{p}>.05$ ).

Discussion/Conclusion: In a rat model, ADSCs yielded increased fusion mass volume and rates of fusion, while BMSCs displayed increased fusion mass volume when combined with a clinical grade bone graft substitute. ADSCs showed a trend towards higher fusion mass volume and rates of fusion compared to BMSCs. Ongoing histological studies will evaluate the quality of bone formed in the fusion masses. 


\section{Paper 37. Fracture of Femoral Ring Grafts Used for ALIF Procedures: A Relatively Common Finding That Did Not Significantly Affect the Rate of Reoperation}

Travis Philipp, MD, Stephanie Radoslovich, BA, Jung Yoo, MD

Oregon Health and Science University, Portland, Oregon

Background/Introduction: Femoral ring allograft (FRA) was one of the first materials used as an anterior vertebral interbody graft. Although still commonly utilized for anterior lumbar interbody fusion (ALIF) procedures, there are several reports of failure of these implants. The goal of this study was to identify the incidence, consequences of, and the variables correlated with, FRA fracture following ALIF.

Materials/Methods: A retrospective cohort study was performed using surgical variables for all patients that underwent ALIF using FRAs at an academic institution over a 10-year period. Post-operative radiographs and computed tomography images were reviewed by both the corresponding and senior authors; a fracture group and no-fracture group were created for comparison. The following variables were analyzed: age, sex, height, weight, BMI, smoking status, graft height, index level, presence of spondylolisthesis, adjunct screw use, reoperation at the index surgery site and length of follow up. Frequency data comparisons were performed using contingency table analysis; comparisons of means were analyzed for continuous variables. A multivariate linear regression model was developed using screw use, graft height $<12 \mathrm{~mm}$, index level and weight as independent variables.

Results: 76 FRAs in 59 patients were identified, 13 (17\%) of which fractured. Mean follow up was 2.76 years. There was no difference in length of follow up between the two groups and there was no difference in reoperation rate between the fracture and no fracture groups $(\mathrm{p}>0.05)$. Age, sex, smoking status, use of buttress screws, weight, index level and presence of spondylolisthesis were not correlated with incidence of fracture $(\mathrm{p}>0.05)$. There was a significant correlation between the height of FRA and the incidence of fracture. Two percent (1/52) of grafts $>12 \mathrm{~mm}$ fractured and $50 \%(12 / 24)$ of grafts $<12 \mathrm{~mm}$ fractured ( $\mathrm{p}$ $<0.0001)$. Using ordinary least squares regression analysis, this result was independent of patient weight, use of buttress screws, and index level.

Discussion/Conclusion: In this series nearly one of every five FRAs used for ALIF procedures fractured, though a fractured graft was not correlated with an increased rate of reoperation. Short graft height $(<$ $12 \mathrm{~mm}$ ) was the only variable correlated with graft fracture. 


\section{Paper 38. Utilization of a Dᄀdimer Protocol for Detection of Deep Vein Thrombosis in Spine Patients}

Ilyas Eli, MD, Mohammed Azab, MD, Jonathan Harper, BS, Michael Karsy, MD, PhD, Jian Guan, MD, Andrea Brock, MD, MSc, William Couldwell, MD, PhD, Erica Bisson, MD, MPH; Andrew Dailey, MD University of Utah, Salt Lake City, UT

Background/Introduction: Venous thromboembolism, comprising deep vein thrombosis (DVT) and pulmonary embolism, is a significant source of mortality for spine patients and remains difficult to diagnose. The use of venous duplex ultrasound (VDU) for confirmation of venous thromboembolism is costly and requires experienced personnel for acquisition and interpretation. We evaluated a novel protocol using levels of D-dimers to screen for DVT.

Materials/Methods: A retrospective bioinformatics analysis identified neurosurgical inpatients who underwent a D-dimer screening protocol and had a VDU study evaluating the presence of DVT from March 2008 through July 2017. The D-dimer screening protocol involved assessing serum D dimer levels at baseline and every other day during hospitalization. Patients with levels $>3.0 \mu \mathrm{g} / \mathrm{ml}$ or that increased over 2 consecutive days underwent a VDU. Clinical risk factors and D-dimer levels were evaluated for prediction of DVT.

Results: Among a total of 1918 patient encounters ( $n=1854$ patients), 506 spine patients were identified with an overall DVT detection rate of $15.4 \%$. A D-dimer of $>2.5 \mu \mathrm{g} / \mathrm{ml}$ on admission conferred sensitivity $=0.68$, specificity $=0.52$, positive predictive value $(\mathrm{PPV})=0.20$, and negative predictive value $(\mathrm{NPV})=0.90$. A D-dimer value of $>3.5 \mu \mathrm{g} / \mathrm{ml}$ during hospitalization predicted DVT risk with a sensitivity $=0.77$, specificity $=0.27, \mathrm{PPV}=0.17$, and $\mathrm{NPV}=0.88$. Multivariable logistic regression showed that after adjusting for age, gender, length of stay, and completion of a major surgical procedure within the last 30 days, a D-dimer level $>2.5 \mu \mathrm{g} / \mathrm{ml}$ on admission conferred an independent risk of DVT (odds ratio=2.23, $95 \%$ confidence interval $=1.30-3.81)$.

Discussion/Conclusion: The use of a D-dimer protocol was effective in screening for DVTs in spine patients. A cutoff of $>2.5 \mu \mathrm{g} / \mathrm{ml}$ at day 0 or $>3.5 \mu \mathrm{g} / \mathrm{ml}$ during admission could be utilized for screening purposes. Additional refinement of this screening model could further improve identification of DVT in a practical and cost-effective manner. 


\section{Paper 39. A Validated Preoperative Score for Predicting 30-day Readmission after 1-2 level Elective Posterior Lumbar Fusion}

Deeptee Jain, $M D^{I}$, Paramjit Singh, $M D^{2}$, Mayur Kardile, $M D^{3}$, Sigurd Berven, $M D^{4}$

${ }^{l}$ New York University, Apt 10D, New York, ${ }^{2}$ University of California San Francisco, San Francisco, CA, ${ }^{3}$ University of California, San Francisco, San Francisco, CA, ${ }^{4}$, San Francisco, CA

Background/Introduction: Posterior lumbar fusion is one of the most commonly performed procedures in the United States. Readmission rates are an important metric of quality of care. The purpose of this study was to develop a model to predict 30-day readmission rates in elective 1-2 level lumbar posterior spine fusion patients.

Materials/Methods: This was a case control study. Patients who underwent elective 1-2 level lumbar PSF in the State Inpatient Database were identified. Data was queried for 30-day readmission, demographic and surgical data. Patients were randomly assigned to either the derivation or validation cohort. Stepwise multivariate analysis was conducted on the derivation cohort to predict 30-day readmission. Next, the Readmission after Posterior Spinal Fusion (RAPSF) score was created. Variables with odds ratio (OR) > 1.1 and $p<0.01$ on multivariate analysis were included; the value assigned to each variable was based on the OR and calibrated to 100. Linear regression was performed between readmission rate and RAPSF score to test its accuracy both in the derivation and validation cohorts.

Results: There were 92,262 and 90,257 patients in the derivation and validation cohorts. Thirty-day readmission rates were $10.9 \%$ and $11.1 \%$ respectively. The RAPSF consisted of (point value in parentheses): age (50-59: 4, 60-69: 4, 70-79:6, >90: 8), gender (female: 3), race (Hispanic: 4, black: 5), insurance (Medicare: 4, Medicaid: 5, other -non-commercial: 3), anterior approach (4), cerebrovascular disease (3), chronic pulmonary disease (3), congestive heart failure (4), diabetes without chronic complications (4), diabetes with chronic complication (4), hemiplegia/paraplegia (7), rheumatic disease (3), drug abuse (4), electrolyte disorder (4), osteoporosis (3), depression (3), obesity (4), morbid obesity (5). Linear regression between readmission rate and RAPSF fit the derivation and validation cohorts with an adjusted $\mathrm{r} 2$ of 0.92 and 0.94 and a coefficient of 0.011 and $0.011(\mathrm{p}<0.001)$, respectively.

Discussion/Conclusion: We developed an easy-to-use tool, the RAPSF, to accurately predict readmission rates in patients undergoing elective 1-2 level posterior lumbar fusion. The RAPSF may be useful to empower informed choice regarding the risks of surgery and to guide an evidence-based approach to preoperative optimization and risk adjustment within alternative payment models for elective spine surgery. 
Paper 40. Does the Mode of Questionnaire Administration Matter for Satisfaction with Outcomes in Lumbar Spine Surgery? A Comparison Study of 32,014 Patients from National Scale Registry

\author{
Inamullah Khan, $M D^{l}$, Jacquelyn Pennings, $P h D^{2}$, Ahilan Sivaganesan, $M D^{l}$, Anthony Asher, $M D^{3}$, \\ Mohamad Bydon, M.D. ${ }^{4}$, Kristin R. Archer, DPT, PhD ${ }^{l}$, Clinton Devin, $M D^{l}$ \\ ${ }^{l}$ Vanderbilt University Medical Center, Nashville, $\mathrm{TN},{ }^{2}$, Nashville, $\mathrm{TN},{ }^{3}$, Charlotte, $\mathrm{NC},{ }^{4}$, Rochester, \\ $M N$
}

Background/Introduction: Lower back pain (LBP) is a leading cause of global disability. Most of these patients need surgical management, and in the past two decades an exponential rise has been noticed in the rates of lower back surgeries. Modern health care emphasizes patient satisfaction; hence the North American Spine Satisfaction (NASS) questionnaire, along with other available instruments, are being utilized in many prospective registries as a measure of outcomes after spine surgery. The NASSquestionnaire can be administered by two modes, namely self-administration and intervieweradministration each having their own pros and cons. In the present study we made an effort to understand the impact of mode of administration on the NASS-questionnaire score in patients who underwent elective surgery for degenerative lumbar diseases.

Materials/Methods: The Quality and Outcomes Database (QOD) lumbar module was queried for patients who had completed their 3-month follow-up. Patients were grouped into either interviewer- or selfadministration based on completion of their patient reported outcomes (PROs) questionnaire via the respective mode at baseline and 3-month. Finally, a regression model was fitted with 3-month posttreatment satisfaction as the outcome. The covariates for this model included mode of questionnaire administration, age, gender, diabetes, anxiety, depression, level of education and surgical approach.

Results: The analysis included 32,014 total patients who underwent elective lumbar spine surgery and had a complete 3-month follow-up. Overall, patients improved in a clinically significant manner on all the PROs [EQ-5D, Oswestry Disability Index (ODI), and pain scores]. Patients who completed the questionnaire via self-administration reported significantly higher pain and disability-scores on follow-up; however, the differences were not clinically meaningful. The regression analysis showed that patients completing the questionnaire via interviewer-administration had higher odds of achieving satisfaction [Odds ratio: 1. 619, $(95 \%-\mathrm{CI}=1.482-1.768), \mathrm{p}=<0.001$ ].

Discussion/Conclusion: Post-treatment satisfaction is known to depend on many factors ranging from patients' socioeconomic and preoperative health-status to factors related to physician-patient interaction, including some of which are not directly related to the quality of care. Our study identifies the mode of questionnaire administration as one of the influencers of patient satisfaction with outcomes, after their lumbar spine surgery. 
Paper 41. Do patients that catastrophize back and leg pain prior to lumbar decompression do worse after surgery?

Sohrab Virk, $M D^{1}$, Jung Mok, $B S^{2}$, Avani Vaishnav, $M B B S^{3}$, Steven McAnany, $M D^{3}$, Todd Albert, $M D^{4}$, Sravisht Iyer, $\mathrm{MD}^{4}$, Catherine Himo Gang, $M P H^{5}$, Sheeraz Qureshi, $M D, M B A^{4}$

${ }^{1}$, New York, New York, ${ }^{2}$ Weill Cornell Medical College, New York, NY, ${ }^{3}$ Hospital for Special Surgery, New York, New York, ${ }^{4}$ Hospital for Special Surgery, New York, NY, ${ }^{5}$, New York, NY

Background/Introduction: The purpose of this study is to examine the role that pre-operative pain catastrophizing might have on post-operative outcomes for these patients.

Materials/Methods: We defined patients catastrophizing pain as having pre-operative VAS leg/back scores of 9 or above. These pain catastrophizing (PC) patients were then divided into a cohort with VAS leg scores above 9 (VL-PC) and a cohort with VAS back scores above 9 (VB-PC). Health related quality of life (HRQOL) scores were collected on these patients in the pre-operative period and the post-operative period up to 1 year out from surgery. These scores included the oswestry disability index (ODI), short form 12 (SF-12) mental health scores (MHS) and physical health scores (PHS) and PROMIS scores. We performed a student's T-test to analyze if there was a difference in outcomes after surgery between VL-PC patients and those that did not catastrophize leg pain (non-VL-PC).

Results: 139 patients were included within our analysis. There were 21 patients in the VB-PC cohort (14 female, mean age - 48.0 +/- 17.6 years old) and 31 patients in the VL-PC cohort (22 female, mean age $49.4+/-18.2$ years old). The pre-operative SF-12 MHS (42.0 vs. $47.5, \mathrm{p}=0.02)$, SF-12 PHS (28.1 vs 32.2, $\mathrm{p}=0.003)$, ODI (59.7 vs 38.0, $\mathrm{p}<0.001)$ and PROMIS (29.3 vs 35.7, p <0.001) scores were worse for the VL-PC cohort versus non-VL-PC. The pre-operative SF-12 MHS (40.6 vs. 47.9, $\mathrm{p}=0.02$ ), SF-12 PHS (28.6 vs 32.1, $\mathrm{p}=0.02)$, ODI (59.5 vs 39.7, $\mathrm{p}=0.01)$ and PROMIS (29.5 vs 35.7, $\mathrm{p}<0.001$ ) scores were worse for the VL-BC cohort versus non-VL-BC. In the post-operative period, the VL-PC cohort had worse HRQOL scores at the 2 week time point in terms of SF-12 PHS (28.1 vs 47.5, p = 0.02) and SF-12 MHS (42.0 vs $47.5, \mathrm{p}=0.02)$.

Discussion/Conclusion: These results show that patients that catastrophize back or leg pain may have short term worse outcomes after lumbar decompressive surgery. This difference in outcomes between those that catastrophize pain and those that do not catastrophize pain appears to disappear after 6 weeks out from surgery. 
Paper 42. Admission NarxCare Narcotics Scores are Not Associated with Adverse Surgical Outcomes or Self-Reported Patient Satisfaction Following Elective Spine Surgery

Anoop Galivanche, B.S. ${ }^{I}$, Michael Mercier, BS ${ }^{l}$, Murillo Adrados, $M D^{2}$, Ryan McLynn, B.S. ${ }^{3}$, Nidharshan Anandasivam, $B S^{l}$, Arya Varthi, $M D^{4}$, Lee Rubin, $M D^{I}$, Jonathan Grauer, $M D^{5}$

${ }^{l}$ Yale School of Medicine, New Haven, CT, ${ }^{2}$ Yale School of Medicine, New Haven, CT , ${ }^{3}$ Yale School of Medicine, New Haven, Connecticut, ${ }^{4}$ Yale University, New Haven, CT, ${ }^{5}$, New Haven, CT

Background/Introduction: The effect of pre-operative narcotics usage on post-operative outcomes and patient satisfaction following spine surgery has been a source of debate in spine surgery literature. The current study investigated how 30-day peri-operative outcomes and post-operative self-reported patient satisfaction differed in patients with varying pre-operative opioid use as measured by admission NarxCare narcotics use scores (opioid misuse scores derived from the state PDMP).

Materials/Methods: Elective spine surgery cases performed at our institution between October 2017 and March 2018 were included in the study. NarxCare narcotics use scores at the time of admission for each patient were extracted from the patient's chart. Additionally extracted were demographic; comorbidity; and 30-day adverse events, readmissions, reoperations, and mortality data. Hospital Consumer Assessment of Healthcare Providers and Systems (HCAHPS) survey data was also aggregated for patients in the study cohort. Multivariate logistic regressions were performed to compare the odds of having an adverse events, readmission, reoperation, and mortality between the different narcotics groups, using patients in the " 0 " narcotics score bin as the referent. Multivariate regressions controlled for demographic factors and preoperative comorbidity burden. ANOVA analyses were performed to compare HCAHPS survey response rates and HCAHPS survey results between the different narcotics score groups.

Results: Of 346 patients who met inclusion criteria for the study, 74 patients had an admission NarxCare narcotics score of 0,58 patients had a score between 1 and 99, 117 patients had a score between 100-299, 76 patients had a score between 300 and 499, and 21 patients had a score that exceeded 500. Multivariate logistic regressions did not detect statistically significant differential odds of experiencing adverse events between the different groups. Analyses of variance did not detect statistically significant differences in HCAHPS survey response rates, total HCAHPS scores, or HCAHP sub-group scores between the different groups.

Discussion/Conclusion: The study was unable to detect differences in 30-day perioperative outcomes or self-reported patient satisfaction between patients of varying admission NarxCare narcotics scores. Although there are many reasons to address preoperative patient narcotic utilization, the current study suggests that surgeons may be able to safely perform elective spine surgery in appropriately selected patients. 
Poster 01. Factors associated with achieving a clinically significant improvement in patients undergoing minimally invasive lumbar decompression surgery

Avani Vaishnav, $M B B S^{l}$, Steven McAnany, $M D^{1}$, Todd Albert, $M D^{2}$, Sravisht Iyer, $M D^{2}$, Catherine Himo Gang, $M P H^{3}$, Sheeraz Qureshi, $M D, M B A^{2}$

${ }^{l}$ Hospital for Special Surgery, New York, New York, ${ }^{2}$ Hospital for Special Surgery, New York, NY, ${ }^{3}$, New York, NY

Background/Introduction: The purpose of this study is to determine demographic factors, surgical variables and pre-operative patient-reported outcome measures (PROMS) associated with achieving an improvement greater than the Minimum Clinically Important Difference (MCID) by 3 months postoperatively in patients undergoing minimally invasive lumbar decompression surgery.

Materials/Methods: A retrospective review of prospectively collected data on patients who underwent minimally invasive lumbar decompression surgery was performed. Patient demographics and operative details were extracted from electronic medical records. Various PROMs, including Oswestry Disability Index (ODI), Visual Analog Scale (VAS) for back pain and leg pain, Short Form-12 Physical and Mental Health Scores (SF-12 PHS and MHS) and PROMIS Physical Function Scores collected pre-operatively and at each follow-up were analyzed. MCID of 12.8 for ODI, as determined by Copay et al was used to assess the number of patients experiencing a clinically significant improvement at each time-point.

Results: A total of 93 patients, with a mean age of 55 years were included in this study. $53.3 \%$ of patients experienced an improvement > MCID by 2 weeks, $56.5 \%$ by 6 weeks and $64.5 \%$ by 3 months postoperatively. Individual regressions showed advanced age $(\mathrm{p}<0.001)$, pre-operative VAS leg pain $(\mathrm{p}=0.007)$, pre-operative ODI $(\mathrm{p}=0.001)$ and pre-operative SF-12 MHS $(\mathrm{p}=0.027)$ to be significant predictors. The multiple regression model demonstrated that when controlling for other variables, younger age [OR: 0.943, $\mathrm{p}<0.001$ ) and higher pre-operative ODI [OR: 1.046, $\mathrm{p}=0.008)$ were the only significant predictors. The results of the logistic regression to predict an improvement $>$ MCID by 3 months was statistically significant $(\mathrm{p}<0.0001)$. The model explained 40.8\% (Nagelkerke R2) of the variance in whether patients had a clinically significant improvement and correctly classified $80.6 \%$ of cases.

Discussion/Conclusion: The results of our study show that age and pre-operative ODI are associated with patients experiencing a clinically significant improvement following minimally invasive lumbar decompression surgery. These findings can be used to pre-operatively identify patients who may experience a longer recovery and counsel them appropriately. Larger studies are warranted to identify additional factors associated with patient recovery. 


\section{Poster 02. Preoperative Depression Affects Health-Care Related Quality of Life after Lumbar Decompression Surgery}

Srikanth Divi, M.D. ${ }^{l}$, Dhruv Goyal, BA ${ }^{l}$, Matthew Galetta, BA ${ }^{l}$, Kristen Nicholson, PhD ${ }^{l}$, Kris Radcliff, $M D^{2}$, Barrett Woods, $M D^{3}$, Alan Hilibrand, $M D^{4}$, Chris Kepler, $M D M B A^{5}$, Alexander Vaccaro, $M D P h D$ $M B A^{6}$, Gregory Schroeder, M.D. ${ }^{4}$

${ }^{1}$, Philadelphia, PA,${ }^{2}$ Rothman, Philadelphia, PA, ${ }^{3}$ Rothan Institute, Philadelphia, Pennsylvania, ${ }^{4}$ Rothman Institute, Thomas Jefferson University Hospital, Philadelphia, PA, ${ }^{5}$, Philadelphia ,PA, ${ }^{6}$ Rothman Institute, Philadelphia, PA

Background/Introduction: The impact of preoperative depression on health-care related quality of life (HRQOL) outcomes after lumbar decompression is not well established. In this study, we used two criteria to identify depression and used it to correlate with patient outcomes.

Materials/Methods: Patients undergoing lumbar decompression between one to three levels at a single center, academic hospital were retrospectively identified. Patients under the age of 18 years, and those undergoing surgery for infection, trauma, tumor, or revision, and less than one year follow-up were excluded. The cohort was split into groups using an existing clinical diagnosis of depression or a score of MCS- $12 \leq 45.6$ on the preoperative SF-12 survey. Absolute HRQOL scores, the recovery ratio and the percent of patients achieving MCID between groups were compared, and a multiple linear regression analysis was performed.

Results: A total of 195 patients were included. Based on MCS-12 score, 59 (30.3\%) had symptoms of depression, and $136(69.7 \%)$ did not. Only $15(8.4 \%)$ had depression based on a preexisting clinical diagnosis. When analyzing by MCS-12 score, preoperative ODI $(\mathrm{p}<.001)$ and VAS Leg $(\mathrm{p}=.018)$ and postoperative PCS-12 ( $\mathrm{p}=.015)$ and ODI ( $\mathrm{p}=.006)$ scores were significantly different between groups, with the depression group exhibiting worse disability at both time-points. Analyzing by preexisting diagnosis, only postoperative ODI ( $\mathrm{p}=.027)$ was different between groups. While both groups improved from baseline as seen by the recovery ratio, PCS-12 scores improved less in patients with the depression by MCS- 12 group ( $\mathrm{p}=.004)$. The percent of patients achieving MCID for ODI was lower for patients with a preexisting diagnosis of depression $(54.5 \%$ vs. $64.8 \%, \mathrm{p}=.046)$. On multiple linear regression analysis, depression by MCS-12 was a significant predictor of PCS-12 (p=.003) and ODI $(\mathrm{p}=.010)$ scores, whereas depression by diagnosis was a predictor of ODI ( $\mathrm{p}=.025)$ and VAS Back scores $(\mathrm{p}=.020)$.

Discussion/Conclusion: Overall HRQOL outcomes improved for both groups after lumbar decompression surgery. However, patients with depression measured by preoperative MCS-12 score or preexisting diagnosis, had more disability perioperatively than those without depression. 
Poster 03. Comparison of Subcutaneous Fat Thickness in the Lumbar Spine Related to BMI Between Males and Females

Christian Klare, $M D^{l}$, Nicholas Johnson, $M D^{2}$, Todd Chapman, $J r$., $M D^{3}$, Bruce Darden, $M D^{4}$, Casey Davidson, $M D^{5}$, Alden Milam, $M D^{2}$, Alfred Rhyne, $M D^{6}$, P. Brad Segebarth, $M D^{4}$, Leo Spector, $M D^{4}$, Eric Laxer, $M D^{2}$

${ }^{1}$, Lebanon, $\mathrm{NH},{ }^{2}$, Charlotte, $\mathrm{NC},{ }^{3}$ Washington University School of Medicine, St. Louis, MO, ${ }^{4}$ OrthoCarolina, Charlotte, NC, ${ }^{5}$ OrthoCarolina Spine Center, Charlotte, $\mathrm{NC},{ }^{6}$ OrthoCarolina, Charlotte, North Carolina

Background/Introduction: Body mass index (BMI) is often used as a factor when identifying patients at increased risk of surgical complications, including postoperative infections. Search of the spine literature demonstrates that increasing subcutaneous fat thickness of the lumbar spine may serve as a more accurate predictor of postoperative infection risk. Our hypothesis was that increasing BMI would lead to an increased fat thickness, and as BMI increased women would have a more rapid increase in fat thickness.

Materials/Methods: A database search of 330 consecutive patients undergoing MRI of the lumbar spine without contrast beginning January of 2018 was performed. Sample collection was equal from 3 different MRI locations in our system in order to support generalizability. Patient information was de identified. Measurements of the subcutaneous fat thickness at the L4-5 level were performed by two evaluators $(\mathrm{CK}, \mathrm{NJ})$ who were blinded to the sex, BMI, and first name of the patient. Measurements were performed on both the axial and sagittal T1 sequences using PACS imaging measurement tools. Patients who did not have MRI performed or did not have imaging adequate for measurement were excluded.

Results: 250 patients, including 137 females (54.8\%) and 113 males (45.2\%) were included in the final analysis. Mean BMI was 28.1 (range $=18.6-48.9$ ), while mean subcutaneous fat thickness measured on axial images was $32 \mathrm{~mm}$ (range $=3.5-64.9$ ). The positive correlation of BMI on subcutaneous fat thickness was stronger for males $(0.77)$ than females $(0.67)$. Females have a significantly $(\mathrm{p}=.0001)$ higher axial depth of $25.54 \mathrm{~mm}$, on average, after adjusting for the effects of BMI. With every increased unit of BMI females had a significantly $(\mathrm{p}=0.0022)$ less increase in fat thickness of compared to males $(0.7 \mathrm{~mm})$.

Discussion/Conclusion: This study demonstrates that increasing BMI is correlated with increasing subcutaneous fat thickness in the lumbar spine, in both males and females. On average females had a greater subcutaneous fat thickness, however increasing BMI had less of an effect on increasing subcutaneous fat thickness compared to males. 


\section{Poster 05. Risk Factors for Postoperative Urinary Tract Infections Following Anterior Lumbar Interbody Fusion}

Danny Lee, $B S^{l}$; Ryan Lee, BA, $M B A^{2}$, Megan Cross, $B S^{l}$, Uchechi Iweala, $M D, M B A^{3}$, Jeffrey Weinreb, $M D^{3}$, David Falk, $M D^{4}$, Warren $Y u, M D^{3}$

${ }^{I}$ George Washington University, Washington, DC, ${ }^{2}$, Washington, District of Columbia, ${ }^{3}$ George

Washington University Department of Orthopaedic Surgery, Washington, DC, ${ }^{4}$ University of

Pennsylvania Department of Orthopaedic Surgery, Philadelphia, PA

Background/Introduction: Background: Many patients undergoing anterior lumbar interbody fusion (ALIF) have indwelling catheters placed to prevent urinary retention. Although the prolonged use of indwelling catheters in the postoperative period has been shown to increase the risk for developing urinary tract infection (UTI), the current literature indicates that the pathogenesis of UTI is multifactorial. There is a lack of literature investigating predictors of UTI in patients who have undergone ALIF. The present study sought to identify preoperative independent risk factors that predisposed patients to postoperative UTI following ALIF so that surgeons can initiate preventative measures to minimize morbidity in the postoperative period.

Materials/Methods: Materials and Methods: The American College of Surgeons-National Surgical Quality Improvement Program (ACS-NSQIP) database was queried to identify 10,232 patients who had undergone ALIF from 2005 to 2016. 144 patients (1.41\%) developed a postoperative UTI while 10,088 patients $(98.59 \%)$ did not. Univariate analyses were conducted to compare the cohorts' demographics and preoperative comorbidities. Multivariate logistic regression models were then utilized to identify significant predictors of postoperative UTI following ALIF while controlling for differences in univariate analyses.

Results: Results: Age $\geq 60$ years $(\mathrm{p}=0.022)$, female sex $(\mathrm{p}<0.001)$, alcohol use $(\mathrm{p}=0.014)$, open wound/wound infections $(\mathrm{p}=0.019)$, and steroid use $(\mathrm{p}=0.046)$ were independent risk factors for postoperative UTI. Longer operative times were also independent predictors for developing UTI: $120 \mathrm{~min} \leq$ $\mathrm{x}<180 \min (\mathrm{p}=0.050), 180 \mathrm{~min} \leq \mathrm{x}<240 \mathrm{~min}(\mathrm{p}=0.025)$, and $\geq 240 \min (\mathrm{p}=0.001)$. Postoperative UTI independently increased the risk for various 30 -day complications including pneumonia, blood transfusions, sepsis, thromboembolic events, and extended length of stay.

Discussion/Conclusion: Conclusions: This study identified age $\geq 60$ years, female gender, alcohol use, steroid use, and open wound/wound infections as independent risk factors for postoperative UTI following ALIF. Identifying these risk factors may improve surgeons' ability to risk stratify patients undergoing ALIF in order to initiate more aggressive prophylactic measures to prevent UTIs and their associated complications. Future work analyzing the efficacy of more stringent quality improvement protocols that taper alcohol/steroid use preoperatively and reducing procedural time with the aim of lowering UTI risk is warranted. Preoperative wound care is strongly encouraged to decrease UTI risk. 


\title{
Poster 06. Immediate Restoration of Lordosis in Single-level Minimally Invasive Transforaminal Lumbar Interbody fusion (MI-TLIF): A comparison of Expandable and Static Interbody Cages
}

\author{
Avani Vaishnav, $M B B S^{l}$, Philip Saville, $M D^{l}$, Steven McAnany, $M D^{l}$, Sertac Kirnaz, $M D^{2}$, Christoph \\ Wipplinger, $M D^{2}$, Rodrigo Navarro-Ramirez, $M D, M S c^{2}$, Roger Härtl, $M D^{2}$, Jingyan Yang, $M H S^{3}$, \\ Catherine Himo Gang, $M P H^{4}$, Sheeraz Qureshi, $M D, M B A^{5}$ \\ ${ }^{l}$ Hospital for Special Surgery, New York, New York, ${ }^{2}$ Weill Cornell Medicine, New York, NY, ${ }^{3}$ Hospital \\ for Special Surgery, New York, New York , ${ }^{4}$, New York, NY, ${ }^{5}$ Hospital for Special Surgery, New York, \\ $N Y$
}

Background/Introduction: Current literature is conflicted regarding the effect of Minimally Invasive Tranforaminal Lumbar Interbody Fusion(MI-TLIF) on sagittal alignment and the role of expandable cage technology. The purpose of this study was to compare generation of lordosis by static and expandable cages, and determine what factors affect segmental lordosis and foraminal decompression.

Materials/Methods: Segmental lordosis(SL), regional lordosis(RL) and Posterior Disc Height(PDH) were measured pre- and post-operatively on upright lateral radiographs of patients undergoing single-level MISTLIF with expandable or static cages. Distance from anterior edge of vertebral body to the front of the cage was measured. Based on pre-operative SL, patients were stratified into: low $(<15 \mathrm{deg})$, moderate $(15-25 \mathrm{deg})$ and high-lordosis $(>25 \mathrm{deg})$. Non-parametric tests were used to compare radiographic parameters between static and expandable cages and the change from pre- to post-operatively. Linear regressions were conducted to assess the effect of pre-operative radiographic parameters, cage-type and cage-position on post-operative SL and PDH.

Results: Of the 171 patients included; 111 were in the static and 60 in the expandable cohort. Patients with low pre-operative lordosis experienced an increase in SL and maintained RL regardless of cage-type. Those with moderate to high pre-operative lordosis experienced a decrease in SL and RL with the static cage but maintained SL and RL with the expandable cage. Although PDH increased in both cohorts, the increase in the expandable cohort was significantly greater. Pre-operative SL was predictive of post-operative SL; and pre-operative SL, pre-operative PDH and cage-type were predictive of post-operative PDH. Cage position was not related to post-operative SL or PDH.

Discussion/Conclusion: Expandable cages showed favorable results in restoring posterior disc height and maintaining lordosis in the immediate post-operative period. Cage position did not impact the restoration of lordosis or posterior disc height. Pre-operative segmental lordosis was the most significant predictor of post-operative segmental lordosis. Thus, pre-operative radiographic parameters and goals of the surgery should be important considerations in surgical planning. 


\section{Poster 07. The Regional Effect of Lumbar Fusion Surgery on Volumetric Bone Mineral Density measured by Quantitative Computed Tomography in Adjacent Vertebrae: A Longitudinal Cohort Study}

Ichiro Okano, $M D^{l}$, Conor Jones, $B A^{2}$, Stephan Salzmann, $M D^{l}$, Courtney Ortiz Miller, $B A^{3}$, Colleen Rentenberger, $M D^{4}$, John Carrino, $M D, M P H^{l}$, Andrew Sama, $M D^{I}$, Frank Cammisa, $M D^{I}$, Federico Girardi, $M D^{5}$, Alexander Hughes, $M D^{1}$

${ }^{l}$ Hospital for Special Surgery, New York, New York, ${ }^{2}$ Hospital for Special Surgery, New York, New York, ${ }^{3}$ Hospital for Special Surgery, New York, New York , ${ }^{4}$, New York, New York, ${ }^{5}$, New york, New York

Background/Introduction: Previous studies reported that lumbar spine surgery has a negative effect on bone mineral density (BMD) in the lumbar spine. Dual X-ray absorptiometry (DXA) was used primarily to evaluate BMD change in these studies, however; DXA has several limitations for measuring BMD in the vertebrae with morphological changes and metal implants. In this study, we investigated the change of BMDs in multiple adjacent vertebrae utilizing quantitative computed tomography (QCT).

Materials/Methods: We reviewed clinical records and images of 296 consecutive patients who underwent posterior lumbar spine surgery between 2014-2017 at a single academic institution. Patients who underwent posterior fusion surgery with instrumentation and had both pre-operative and secondary postoperative computed tomography of the lumbar spine between 6 to 12 months were included. Volumetric bone mineral density (vBMD) measurements by QCT were conducted in L1 to S1 vertebral trabecular bone. The vBMD changes in the vertebrae one or two-level above the upper instrumented vertebra (UIV+1, UIV+2) and one level below the lower instrumented vertebra $(L I V+1)$ were analyzed.

Results: A total of 91 patients met inclusion criteria. Mean age ( \pm SD (range)) was $61.9 \pm 11.9 .55 .6 \%$ of the patients were female. Median interval between the surgery and the secondary CT was 248 (180-364) days. Volumetric BMD $( \pm \mathrm{SD})$ in UIV+1 was $113.1 \pm 32.6 \mathrm{mg} / \mathrm{cm} 3$ preoperatively and $102.4 \pm 32.2$ $\mathrm{mg} / \mathrm{cm} 3$ (percent change:-10.5 $\pm 12.6 \%$ ) postoperatively $(\mathrm{p}<0.001$ ). This trend of $\mathrm{vBMD}$ change was similar regardless of UIV level and number of levels fused. The change of vBMDs in UIV+2 and LIV+1 appeared similar as UIV+1 (UIV+2: $-11.9 \pm 12.3 \%, \mathrm{p}=0.19$, LIV $+1:-7.2 \pm 20.7 \%, \mathrm{p}=0.40)$.

Discussion/Conclusion: Posterior lumbar fusion surgery negatively affected the regional vBMDs measured by QCT in adjacent levels. BMDs in UIV+1, UIV+2, and LIV+1 were equally affected by the surgery. Our finding suggests that the postoperative decrease of regional BMDs in the lumbar spine occurs mainly in the early postoperative phase (within 6 months) and a systemic effect has a greater role, rather than a regional mechanical one, in this postoperative BMD decline. 


\section{Poster 08. Comparative Effectiveness of Expandable versus Static Lateral Lumbar Interbody Fusion Devices: Two Year Clinical and Radiographic Outcomes}

Richard Frisch, $M D^{I}$, Torrey Shirk, $B A^{2}$, Jessica Riggleman, $B S^{2}$, Ingrid Luna, MPH ${ }^{3}$; Charles Ledonio, $M D^{2}$

${ }^{I}$ Southeastern Spine Institute, Mt Pleasant, SC, ${ }^{2}$ Globus Medical, Inc., Audubon, PA, ${ }^{3}$ Globus Medical, Audubon, PA

Background/Introduction: Minimally invasive (MIS) lateral lumbar interbody fusion (LLIF) procedures using large interbody static spacers may require excessive trialing and forceful impaction, leading to iatrogenic endplate disruption, excessive neural retraction, and implant subsidence. The in situ expansion capability offered by expandable interbody spacers facilitates insertion to help reduce endplate damage and optimize endplate contact. This study reported radiographic and clinical outcomes of static and expandable interbody spacers following MIS LLIF, and device-related complications including implant subsidence.

Materials/Methods: This study included 48 patients with degenerative disc disease who underwent MIS LLIF at 1-2 contiguous level(s) using a polyether-ether-ketone static or titanium expandable intervertebral spacer. Twenty-one patients were treated with static spacers, and 27 with expandable spacers. All spacers had supplemental posterior stabilization. Clinical and radiographic outcomes were analyzed at 12 and 24 months postoperatively.

Results: Mean patient age was $66.6 \pm 9.7$ years, $72.9 \%$ of patients were female. No significant differences were observed between static and expandable groups in operative time $(64.0 \pm 39.2 \mathrm{~min}, 73.7 \pm 39.7 \mathrm{~min})$, estimated blood loss $(35.6 \pm 29.2 \mathrm{cc}, 40.0 \pm 30.8 \mathrm{cc})$, or length of hospital stay ( $2.0 \pm 1.3 \mathrm{days}, 2.4 \pm 1.3$ days $)$, respectively $(\mathrm{p}<0.05)$. Mean VAS pain and ODI, and RAND 36 scores improved significantly $(\mathrm{p}<0.05)$ at 24-month follow-up. Patients treated with expandable implants were found to have significantly lower scores for VAS back pain $(\mathrm{n}=39,1.3 \pm 1.7,3.8 \pm 3.0, \mathrm{p}<0.05)$ VAS leg pain $(\mathrm{n}=40,1.4 \pm 2.3,3.6 \pm 3.6, \mathrm{p}<0.05)$, and ODI at 24-month follow-up $(\mathrm{n}=42,13.8 \pm 14.2,27.9 \pm 19.4, \mathrm{p}<0.05)$ compared to static implants. Intervertebral disc height increased significantly $(\mathrm{p}<0.05)$ from baseline for expandable and static groups at each followup through 24 months. At 12-month follow-up the static group had significantly higher subsidence rates. No new subsidence cases developed between 12-month and 24-month follow-up.

Discussion/Conclusion: In this cohort, clinical use of expandable interbody spacers resulted in better outcomes through 24-month follow-up compared to static interbody spacers, with expandable group showing significant improvements in pain and disability than the static group at 24 months. 
Poster 09. Home-Discharge after Elective 1-to-2 level Posterior Lumbar Fusions: Risk factors for Adverse Events and Readmission

Jack Xie, $M S^{l}$, Romi Xi, $M A^{l}$; Azeem Malik, MBBS ${ }^{2}$, Elizabeth $Y u, M D^{3}$, Jeffery Kim, $M D^{4}$, Safdar Khan, $M D^{5}$

${ }^{1}$, Columbus, Ohio, ${ }^{2}$ The Ohio State University Wexner Medical Center, Columbus, Ohio, ${ }^{3}$ Ohio State University, Columbus, Ohio, ${ }^{4}$ The Ohio State University Wexner Medical Center, Columbus, OH, ${ }^{5}$, Columbus, $\mathrm{OH}$

Background/Introduction: With a shift towards value-based payment systems, spine surgeons have been increasing the number of home discharges in order to decrease the overall healthcare strain. However, the current method does not discern potential high-risk groups more prone to be affected by post-surgical complications and who may require more attentive follow-up.

Materials/Methods: The 2012-2016 ACS-NSQIP databased was utilized for patients undergoing elective 1-to-2 level posterior lumbar fusions using CPT codes $(22612,22630$, and 22633). Patients discharged to a destination other than home were removed from the study sample. Patients undergoing fusion for deformity, malignancy and/or fractures were also removed from the study sample.

Results: Home-discharged patients had an overall readmission rate of $4.4 \%$ and re-operation rate of $2.5 \%$. Of the total 19,179 home-discharged patients who were included in the study, 546 (2.8\%) experienced any adverse event (AAE), 276 (1.4\%) experienced severe adverse events (SAE), and 321 (1.7\%) experienced minor adverse events (MAE). Multi-variate analysis identified the following predictors associated with experiencing an AAE, listed in order of descending calculated odds ratios: bleeding disorder (OR 2.25), $\mathrm{BMI} \geq 35.0$ vs. $\mathrm{BMI}<25$ (OR 1.96), chronic steroid use (OR 1.89), a LOS $>3$ days (OR 1.53), insulindependent diabetes mellitus (OR 1.44), hypertension (OR 1.28) and female gender (OR 1.24). Readmission for home-discharge patients were associated with the following predictors: pre-discharge complication (OR 2.12), bleeding disorder (OR 1.84), chronic steroid use (OR 1.55), age $>75$ (OR 1.49), age $>65$ (OR 1.26), history of severe COPD (OR 1.43), total operative time $>210 \mathrm{~min}$ (OR 1.26), ASA $>$ II (OR 1.26) and undergoing 2-level fusion. Furthermore, the predictors of re-operation were determined to be pre-discharge complication (OR 2.22), bleeding disorder (OR 1.89), chronic steroid use (OR 1.60), ASA >II (OR 1.40), operative time $>210$ mins (OR 1.28) and length of stay $>3$ days (OR 1.25).

Discussion/Conclusion: Utilizing the ACS-NSQIP database, the current study identifies and risk-stratifies adverse events, readmissions, and reoperations amongst home-discharge patients undergoing posterior lumbar fusion. Given both the increasing complexity of spine surgeries and the shift to a value-based healthcare model, this study serves as a framework for establishing a more expenditure-conscientious model for determining preferred supplemental post-operative support. 
Poster 11. Minimally Invasive Transforaminal Lumbar Interbody Fusion (MI-TLIF) vs Minimally Invasive Lateral Lumbar Interbody Fusion (MI-LLIF): A comparison of immediate post-operative outcomes for the assessment of decompression

\author{
Avani Vaishnav, $M B B S^{1}$, Steven McAnany, $M D^{l}$, Todd Albert, $M D^{2}$, Sravisht Iyer, $M D^{2}$, Catherine Himo \\ Gang, $M P H^{3}$, Sheeraz Qureshi, $M D, M B A^{2}$ \\ ${ }^{l}$ Hospital for Special Surgery, New York, New York, ${ }^{2}$ Hospital for Special Surgery, New York, NY, ${ }^{3}$, \\ New York, NY
}

Background/Introduction: Surgical decompression to relieve pressure on neural structures can be performed by direct decompression (i.e. resection of impinging tissue), indirect decompression (i.e. distraction between vertebrae to increase the space), or a combination of the two. The purpose of this study is to compare outcomes of surgical procedures that involve direct and indirect decompression (i.e. MITLIF) to procedures that involve only indirect decompression (i.e. MI-LLIF), to assess if the additional direct decompression in MI-TLIF results in better outcomes.

Materials/Methods: Clinical outcomes, including length of stay, post-operative pain, in-hospital complications and need for additional direct decompression surgery, and patient-reported outcomes (PROs), including ODI, VAS and SF-12 in patients undergoing MI-TLIF and MI-LLIF were compared using chi-square test for categorical and student's t-test for continuous variables. Mixed ANOVA was used to assess if the change in PROs from pre-operatively to post-operatively was different between MI-TLIF and MI-LLIF.

Results: Of the 72 patients, 49 had MI-TLIF and 23 had MI-LLIF. There were no significant differences in age $(\mathrm{p}=0.255)$, sex $(\mathrm{p}=0.157)$ or BMI $(\mathrm{p}=0.933)$. The number of levels operated was greater in the MI-LLIF group compared to MI-TLIF (1.43vs 1.12, $\mathrm{p}=0.047$ ), with almost $90 \%$ of MI-TLIFs being 1-level procedures and none 3-level. In contrast, $65 \%$ of MI-LLIFs were 1-level, 26\% 2-level and $8.7 \%$ 3-level procedures. The level(s) operated were different, with over $60 \%$ of MI-TLIFs at L4-L5 and 34.5\% at L5S1, compared to 51.6\% of MI-LLIFs at L3-L4, 24.2\% at each L2-L3 and L4-L5, and none at L5-S1. There was no significant difference in procedural time $(\mathrm{p}=0.281)$, length of stay $(\mathrm{p}=0.216)$, post-operative pain $(\mathrm{p}=0.180)$, or in-hospital complications $(\mathrm{p}=0.384)$.

Discussion/Conclusion: There was no difference in outcomes between those who underwent MI-LLIF (indirect decompression), and those who underwent MI-TLIF (indirect and direct decompression). Thus, in an appropriately selected patient, indirect decompression can provide results that are comparable to direct compression. Underlying pathology, operative level and goals of the surgery should be kept in mind when selecting the procedure. 


\section{Poster 12. To Fuse or Not to Fuse: A 17-Year Spondylolisthesis Review}

Akshay Yadhati, $M D^{l}$, Natasha Greene, B.A. ${ }^{2}$, Chase Grover, $B S^{2}$, Angela Presson, PhD ${ }^{3}$, Tyler Bardsley, $M S^{4}, W$. Ryan Spiker, $M D^{3}$, Brandon Lawrence, $M D^{3}$, Darrel Brodke, $M D^{3}$; Nicholas Spina, $M D^{2}$

${ }^{I}$ SUNY Upstate Medical University, Syracuse, NY, ${ }^{2}$ University of Utah, Salt Lake City, Utah, ${ }^{3}$, Salt Lake City, Utah, ${ }^{4}$ University of Utah, Salt Lake City, UT

Background/Introduction: Recent publications have questioned lumbar fusion over decompression alone as the gold standard in the treatment of lumbar degenerative spondylolisthesis. Proponents of fusion argue for a more enduring solution by providing stabilization and limiting recurrent stenosis at the treated level. Conversely, isolated decompressions achieve the same neurologic relief without limiting motion, potentially reducing the rate of adjacent segment disease. Historically, decompressions have exhibited a higher revision rate, often necessitating fusion. However, decompression techniques become continually more sophisticated and potentially less destructive, influencing these outcomes. A clear consensus is currently lacking in the optimal treatment for degenerative lumbar spondylolisthesis. This study provides an updated analysis of measurement trends in both types of surgery and revision rates.

Materials/Methods: A retrospective, single-center review of all patients undergoing single- level laminectomies from 2000-2017 for spondylolisthesis. Of that cohort, patients were categorized into decompression surgeries with or without the addition of a fusion. Revision surgeries were recorded and demographic information was obtained. Comparisons were performed to identify trends in the patient cohort according to initial treatment type.

Results: Over this 17-year period, 150 patients underwent laminectomy alone, 22 of which then underwent a subsequent revision surgery (14.7\%). The majority of the revision procedures were fusions. On univariate analysis, one degree increase in average facet angle was associated with a 5\% decrease in the hazard of revision $(\mathrm{HR}=0.95,95 \% \mathrm{CI}$ : $0.90-1.00 ; \mathrm{p}$-value $=0.0448) .255$ patients underwent a laminectomy and fusion, 33 of which then underwent a revision surgery (12.9\%). The majority of the revision surgeries are extensions into multilevel constructs. Pelvic incidence, disc angulation changes, and degree of dynamic motion on flexion and extension films did not correlate with failure.

Discussion/Conclusion: In our experience, there was no statistically significant difference in the rate of revision in either laminectomy alone versus laminectomy and fusion patients. Conversion to a fusion, either single or multilevel, in the laminectomy group accounted for the majority of revision procedures compared to adjacent segment degeneration in the fusion cohort. Facet angulation should be carefully scrutinized when considering laminectomy alone in the treatment of degenerative spondylolisthesis. 
Poster 13. EHR-Based Retrospective Analysis of Patients Undergoing Long-Segment Lumbar Spine Fusion a Single Academic Institution Yields Patient-Specific Prediction of Length of Stay after Surgery

Thomas Peterson, $P h D^{l}$, Deeptee Jain, $M D^{2}$, Dmytro Lituiev, $P h D^{3}$, Dexter Hadley, PhD ${ }^{3}$, Sigurd Berven, $M D^{4}$, Atul Butte, $M D, P h D^{3}$

${ }^{l}$ University of California, San Francisco, San Francisco, CA,${ }^{2} \mathrm{New}$ York University, Apt 10D, New

York, ${ }^{3}$ University of California, San Francisco, San Francisco, CA, ${ }^{4}$, San Francisco, CA

Background/Introduction: Background: With the advent of Electronic Health Records (EHRs), the number of patients available for analyzing common and rare medical procedures is increasing, rivaling that of even the largest prospective datasets. Spinal fusion surgery for adult spinal deformity (ASD) is an increasingly commonplace surgery, for which studies using large datasets have largely been based on administrative claims. In this study, we replicate for the first time using comprehensive EHR data to study length of stay (LOS) after long segment lumbar spine fusion.

Materials/Methods: Methods: Structured data elements were extracted from the EHR at a single institution, including demographic variables, ICD-10 diagnosis codes, and medications. All patients undergoing posterior lumbar spine fusion including 3 more levels were identified using CPT codes and were included. We perform logistic factor, multivariate linear, machine learning (lasso) analyses to study factors influencing length of stay in hospital after spinal fusion surgery.

Results: Results: 615 patients were included. Average length of stay was 6.43 days. The following variables were associated with increased LOS: comorbidities, including chronic kidney disease (beta: 2.1) and history of major adverse cardiovascular events (beta: 0.98), socioeconomic factors, female gender (beta: 0.88). Antihyperlipidemic medication was associated with reduced LOS (beta: -0.74 ). The predictive model for LOS had a correlation of 0.390 (p-value: < 0.001.)

Discussion/Conclusion: Conclusion: In this study, we present the first analysis and prediction method using EHR data for length of stay in hospital after long segment posterior lumbar fusion. We expect this analysis to serve as a framework for future studies using EHR datasets, adding new knowledge and external validation to traditional studies. 
Poster 14. Cannabinoid Receptor Agonist has no Adverse Impact on Osteogenic Differentiation or Rat Spinal Fusion

Soyeon Jeong, $M S^{l}$, Chawon Yun, PhD ${ }^{2}$, Mitchell Hallman, B.A. ${ }^{3}$, David Ellenbogen, B.A. ${ }^{4}$, Allison Greene, B.S. ${ }^{5}$, Jonathan Yamaguchi, B.S. ${ }^{4}$,Vivek Shah, B.S. ${ }^{4}$, Stuart Stock, PhD ${ }^{6}$, Wellington Hsu, M.D. ${ }^{7}$, Erin Hsu, $P h D^{8}$

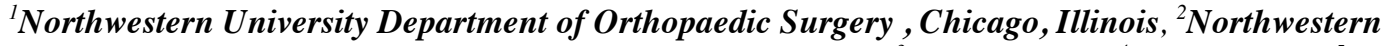
University Department of Orthopaedic Surgery, Chicago, IL, ${ }^{3}$, Raleigh, $N C,{ }^{4}$, Chicago, IL , ${ }^{5}$, Chicago, Illinois, ${ }^{6}$ Northwestern University, Chicago, Illinois, ${ }^{7}$ Northwestern Memorial Hospital, Chicago, Illinois, ${ }^{8}$ Northwestern University Department of Orthopaedic Surgery, Chicago , IL

Background/Introduction: Patients undergoing orthopaedic procedures are at high risk for opioid abuse, making alternative pain management of great public interest. WIN55.212-2 (WIN55) is an agonist of both Cannabinoid Receptors 1 and 2 (CB1 and CB2) and may regulate post-operative pain. Moreover, the endocannabinoid system is thought to play an important role in bone homeostasis. It therefore represents an interesting target for addressing pain without negatively impacting one formation/healing. In this study, we aimed to determine whether WIN55 has a quantifiable impact on osteogenic differentiation and rat spine fusion.

Materials/Methods: Rat bone marrow-derived stromal cells (rBMSCs) were exposed to DMSO vehicle control, $0.2 \mu \mathrm{M}$ or $2 \mu \mathrm{M}$ WIN55. Expression levels of osteogenic marker genes (Alp and Runx2), Alkaline phosphatase (ALP) activity, and matrix mineralization were quantified. Forty-five female Sprague-Dawley rats underwent posterolateral fusion at L4-5 with bilateral placement of an absorbable collagen sponge loaded with $0.5 \mu \mathrm{g}$ of recombinant human bone morphogenetic protein-2. Animals then received intraperitoneal injections for 5 days post-operatively of $0 \mathrm{mg} / \mathrm{kg}$ (ie, DMSO vehicle), $0.5 \mathrm{mg} / \mathrm{kg}$, or 2.5 $\mathrm{mg} / \mathrm{kg}$ of WIN55 (N=15/group). Four weeks postoperatively, spines were harvested for evaluation via radiography, blinded manual palpation-based fusion scoring, and micro-CT imaging for quantification of newly formed bone. The fusion bed was visualized using H\&E/Alcian blue/Orange G staining.

Results: In vitro, exposure to WIN55 did not inhibit gene expression (data not shown), ALP activity (Figure 1A) or matrix mineralization (Figure 1B) of rBMSCs. Similarly, no significant differences among groups were found in regard to fusion scores (data not shown), fusion rates (Figure 1C), new bone formation (Figure D), volumetric analysis (Figure E), and representative bridging bone or lack thereof via histology (data not shown) in the rat spine fusion model.

Discussion/Conclusion: Altogether, our data demonstrated that WIN55 does not adversely affect osteogenic differentiation and spinal fusion outcomes. These results support the notion that endocannabinoids may present a valid alternative to the administration of post-operative opioids following orthopaedic surgeries by reducing pain without inhibiting bone healing. 


\section{Poster 15. Preoperative PROMIS Scores Predict Postoperative Outcomes in Lumbar Spine Surgery Patients}

Joseph Snavely, $M D^{l}$, Joseph Weiner, $B S^{2}$, Daniel Johnson, $M D^{l}$, Wellington Hsu, M.D. ${ }^{3}$, Alpesh Patel, $M D, F A C S^{l}$

${ }^{l}$ Northwestern University, Chicago, IL, ${ }^{2}$ Northwestern University Department of Orthopaedic Surgery, Chicago , IL, ${ }^{3}$ Northwestern Memorial Hospital, Chicago, Illinois

Background/Introduction: The Patient-Reported Outcomes Measurement Information System (PROMIS) is a computer adaptive testing (CAT) system that has been validated in spine surgery patients. PROMIS allows for more efficient and personalized data collection compared to legacy assessment tools. Our objective was to examine the ability of preoperative PROMIS scores to predict postoperative achievement of a minimal important difference (MID) in outcome scores following lumbar spine surgery.

Materials/Methods: 138 patients who underwent lumbar spine surgery at a single institution completed PROMIS Physical Function (PF) and Pain Interference (PI) scores at baseline preoperatively and at 3,12, and 24 months postoperatively. Univariate and multivariate analyses of PROMIS scores and clinical factors were performed. Receiver operating characteristic (ROC) curves were calculated to determine the ability of preoperative scores to predict postoperative achievement of an MID of 8 which has been previously shown in this population. Cutoff values for PF and PI were determined.

Results: Preoperative PROMIS PF and PI scores had a significant effect on achieving postoperative MID after multivariate analysis. Patients with worse preoperative scores were more likely to achieve MID. Both preoperative PF and PI scores showed strong predictive value in determining ability to achieve postoperative MID with respective area under the curve (AUC) of 0.85 and 0.82 . A preoperative PF cutoff T-score of 31.6 had a $64 \%$ chance of achieving postoperative MID, while a preoperative score of 42.5 had a $92.6 \%$ probability of not achieving postoperative MID. A preoperative PI cutoff T-score of 67.8 had an $86 \%$ chance of achieving postoperative MID, while a preoperative score of $58.5 \mathrm{had}$ an $80.1 \%$ probability of not achieving postoperative MID.

Discussion/Conclusion: Preoperative baseline PROMIS PF and PI scores predicted improvement in postoperative outcomes in lumbar spine surgery patients as worse preoperative scores correlated to increased improvement postoperatively. The calculated cutoff T-scores showed the ability to predict which patients would achieve meaningful postoperative improvement. These findings demonstrate how preoperative PROMIS data can be utilized in surgical decision-making and improved patient education regarding postoperative outcomes and expectations. 


\section{Poster 16. Complication Risk in Primary and Revision Minimally Invasive Lumbar Interbody Fusion: A Comparable Alternative to Conventional Open Techniques?}

Cole Bortz, $B A^{l}$; Michael Gerling, $M D^{2}$, Frank Segreto, $B S^{l}$, Samantha Horn, $B A^{l}$, Avery Brown, $B S^{l}$, Katherine Pierce, $B A^{l}$, Haddy Alas, $B A^{3}$, Themistocles Protopsaltis, $M D^{4}$, Aaron Buckland, $M D^{4}$, Peter Passias, $M D^{l}$

${ }^{1}$, New York, NY, ${ }^{2}$ NYU LMC School of Medicine, TriBeCa, NY, ${ }^{3}$ SUNY Downstate College of Medicine, Brooklyn, NY, ${ }^{4}$ Department of Orthopedic Surgery, NYU Langone Orthopedic Hospital, NYU Langone Health, Manhattan, NY

Background/Introduction: Recent studies show similar clinical outcomes between primary and revision lumbar interbody fusion procedures performed using conventional open techniques. As an increasing number of lumbar fusions are performed using minimally invasive surgical techniques (MIS), it is important to assess the corresponding complication risks between primary and revision lumbar interbody fusion procedures.

Materials/Methods: Patients $>18 \mathrm{yr}$ undergoing primary and revision lumbar interbody fusion were propensity score matched for comorbidities, levels fused. Cases were grouped: MIS, open. Patient factors, surgical factors, and periop complication rates were compared between primary and revision cases using means comparison tests.

Results: Included: 154 patients( $62 \pm 12 \mathrm{yr}, 62 \% \mathrm{~F}) ; 29 \%$ of cases were MIS, $71 \%$ were open. Proportion of revision cases by technique: $40.9 \%$ MIS and $53.6 \%$ open. Table 1 shows differences in demographics, operative factors, and clinical outcomes between primary and revision cases for both MIS and open techniques. Primary/revision cases in MIS and open groups did not differ in surgical approach(p $>0.05)$. Primary MIS cases had higher intraop complication rates compared to revisions( $19 \%$ vs $0.0 \%$ ), unlike open procedures, in which primary cases had lower intraop complication rates( $0 \%$ vs $7 \%)$. MIS and open groups did not differ in primary surgery op time $(279 \mathrm{~min}$ vs $280, \mathrm{p}=0.976$ ), but MIS revisions were shorter than open (244 min vs $296, \mathrm{p}=0.024)$. For both primary and revision procedures, there were no differences between MIS and open groups in postop complication rates( $p>0.05)$. Primary MIS cases had higher intraop complication rates than primary open $(19 \%$ vs $0 \%, \mathrm{p}=0.001)$.

Discussion/Conclusion: Clinical outcomes of revision MIS lumbar interbody fusion were similar to those of primary surgery, with no differences in op-time, LOS, EBL, or postop complication rates. Despite higher intraop complication rates for primary MIS fusions, postop clinical outcomes were similar between MIS and open groups for both primary and revision surgery. These results suggest MIS lumbar interbody fusion may be a comparable alternative to conventional open revision. 


\section{Poster 17. A 5-year Review of Hospital Costs and Reimbursement in the Surgical Management of} Degenerative Spondylolisthesis

Keith Lyons, $M D^{l}$, Christian Klare, $M D^{2}$, Mike Bao, BSc ${ }^{3}$, Samuel Kunkel, $M D, M S^{2}$, Adam Pearson, $M D$, $M S^{2}$, William $A b d u, M D, M S^{4}$

${ }^{l}$ Dartmouth-Hitchcock Medical Center, Lebanon, $\mathrm{NH},{ }^{2}$, Lebanon, $\mathrm{NH},{ }^{3}$ Dartmouth-Hitchcock Medical Center, 03766, Lebanon, ${ }^{4}$ Dartmouth-Hitchcock Medical Center, Lebanon, $\mathrm{NH}$ 03766, $\mathrm{NH}$

Background/Introduction: Surgical management of degenerative spondylolisthesis (DS) with spinal stenosis (SS) remains controversial. This study evaluated different charges, expenses, reimbursement, and hospital margins with non instrumented fusion in situ (PLF), posterolateral fusion with pedicle screws (PPS), and PPS with interbody device (PLIF).

Materials/Methods: A retrospective chart review was performed from 2010-2014 based on ICD-9 diagnoses of DS with SS in patients undergoing single level fusions. All service and procedural charges (what was billed to insurance), expenses (direct costs incurred by the hospital), hospital reimbursement, and hospital margins were obtained through the financial audit for each surgery and hospital stay. A multivariate linear regression model was used to compare patient demographics, charges, expenses, etc. A one-way analysis of variance (ANOVA) with Tukey post hoc analysis was used to analyze different reimbursements and margins based upon insurance types.

Results: 233 patients met inclusion criteria for the study. There were no significant differences with BMI, ASA class, length of stay, or gender distribution. Both types of instrumented fusions had longer operating room times and estimated blood loss $(\mathrm{P}<0.001)$. The overall mean charges and expenses for PLF were significantly less compared to both types of instrumented fusions $(\mathrm{p}<0.0001)$. The mean total charges to insurance companies for PLF, PPS, and PLIF were $\$ 74,650, \$ 93,371$, and $\$ 102,766$, respectively. The mean total expenses for the hospital for PLF, PPS, and PLIF were $\$ 13,248, \$ 18,104$, and $\$ 19,740$, respectively. Medicare and private insurance were the most common insurance types; Medicare and private insurance mean reimbursements for PLF were $\$ 36,903$ and $\$ 47,086$, for PPS $\$ 37,450$, and $\$ 53,851$, and for PLIF $\$ 40,171$ and $\$ 51,640$, respectively. Hospital margins for PPS and PLIF in Medicaid patients were negative $(-\$ 3,702$ and $-\$ 6,456)$. Hospital margins were largest for both Worker's Compensation and private insurance patients in all fusion groups. Hospital margins with Medicare for PLF, PPS, and PLIF were $\$ 24,347, \$ 19,205$, and $\$ 23,046$, respectively. Hospital margins for private insurance for PLF, PPS, and PLIF were $\$ 37,569, \$ 36,834$, and $\$ 33,134$, respectively.

Discussion/Conclusion: As more instrumentation is used, the more it costs both the hospital and the insurance companies; hospital margins did not increase correspondingly. 


\section{Poster 18. Molecular Analysis and Proposed Signaling Pathway Involved in Intervertebral Disc Regeneration in Vivo}

\section{Mick Perez-Cruet, $M D$ \\ , Royal Oak, Michigan}

Background/Introduction: Several studies have attempted to use cell therapy for the regeneration of the degenerated intervertebral disc with encouraging but limited success. However, in order to determine the best method for intervertebral disc regeneration, an understanding of the biological, molecular and genetic process of disc regeneration is needed. The current study investigated the process of disc regeneration using immunotracking and expression of human-specific markers in an In-vivo rabbit model of degenerated disc disease (DDD) implanted with human umbilical cord nucleus pulposus(NP)-like cells. To explore the underlying molecular mechanism involved in the ability of NP-like cells to regenerate nucleus pulposus in Vivo, various signaling pathways that play a role in the differentiation process were investigated.

Materials/Methods: A rabbit model of DDD via needle puncture was used. NP-like cells were injected into the degenerated disc determined on pre-injection MRI at two-weeks post needle puncture. Transcriptional analysis was conducted using quantitative real-time reverse transcriptional polymerase chain reaction (qRT-PCR). Cellular RNA was isolated using GeneJet RNA purification kit. Isolated RNA was used to synthesize cDNA. All reactions were prepared in triplicate and normalized to reference genes.

Results: Transcriptional analysis using qRT-PCR of the implanted intervertebral disc revealed that expression of genes involved in the TGFB/Smad pathway. The expression of several intermediates of the TGFB/Smad pathway could be the reason for the production of enhanced levels of glycosaminoglycans (sGAG) and water contents as well as extracellular matrix proteins in degenerated disc injected with stem cell based NP-like cells. TGFB signaling appears to occur by the binding of TGFBR1 to TGFBR2 that triggered the up-regulation of SMADs.

Discussion/Conclusion: A novel molecular mechanism involved in the functioning of human umbilical cord NP-like cells for the regeneration of the degenerated intervertebral disc is proposed. Further investigation is needed to determine the signaling mechanisms in this pathway. The results further provide impetus and basis for clinical trials for treating DDD using human umbilical cord NP-like cells. 


\section{Poster 19. Short- to Medium-Term Follow-up of Lateral Lumbar Interbody Fusion as a Treatment for Adjacent Segment Disease}

Anneliese Heiner, PhD, Jim Youssef, MD; Douglas Orndorff, MD, Juliana Hill, MPH

Spine Colorado, Durango, $\mathrm{CO}$

Background/Introduction: There are few published studies on the results of lateral lumbar interbody fusion (LLIF) as a treatment for adjacent segment disease (ASD), and average follow-up periods of those studies were mostly two years or less. Studies evaluating the longer-term safety and efficacy of LLIF as a treatment for adjacent segment disease would contribute to evidence-based medical decisions for treating this condition. The purpose of this study is to report the short- to medium-term follow-up of patients who underwent LLIF as a treatment for ASD.

Materials/Methods: Data were retrospectively collected from 96 LLIF surgeries in 86 patients to treat ASD, performed at a single private spine practice between October 2006 and March 2016. Surgeries within that time period were excluded when the patient was lost to follow-up at less than two years' postoperatively. Also excluded were surgeries that involved one or more levels with another interbody fusion approach (such as PLIF or TLIF). Seven (7.3\%) of the 96 surgeries were one-level standalone LLIF (LLIF-SA); ten $(10.4 \%)$ were one-level LLIF with supplemental lateral instrumentation (LLIF+LAT); fiftythree $(55.2 \%)$ were one-level LLIF with posterior spinal fusion (LLIF+PSF-1), and twenty-six (27.1\%) were multilevel LLIF with posterior spinal fusion (LLIF+PSF-M). Survival for avoiding a subsequent ASD operation was estimated using the Kaplan-Meier method.

Results: Average age at surgery was 67.6 years (standard deviation 8.9 years), and $53(55.2 \%)$ of the surgeries were performed on female patients. Average follow-up time was 3.75 years (range 76 days-12 years). Thirty (31.3\%) subsequent ASD operations were performed. The overall Kaplan-Meier survival estimate for avoiding a subsequent ASD operation after LLIF as a treatment for ASD was 94.8\% at one year, $87.5 \%$ at two years, $65.1 \%$ at five years, and $52.8 \%$ at ten years. Survival estimates varied by type of surgery (Figure).

Discussion/Conclusion: The subsequent operation rates from this study compare favorably with those from previous shorter-term studies of LLIF as a treatment for ASD and provide more evidence for the use of LLIF as a treatment for ASD. 


\section{Poster 21. Impact of Post-Discharge Fragmentation on Outcomes and Resource Utilization in Lumbar Spine Surgery}

Andrew Pugely, $M D^{l}$, Nathan Hendrickson, $M D, M S^{2}$, Yubo Gao, PhD ${ }^{2}$, Meic Schmidt, MD ${ }^{3}$, Christian Bowers, $M D^{3}$, Anil Nanda, $M D, M P H^{4} ;$ Piyush Kalakoti, $M D^{5}$

${ }^{1}$, New York, NY, ${ }^{2}$ Department of Orthopedics and Rehabilitation, University of Iowa, Iowa City, IA, ${ }^{3}$ New York Medical College, Westchester Medical Center \& Health Network, Valhalla, NY, ${ }^{4}$ RutgersNew Jersey Medical School \& Robert Wood Johnson Medical School, Newark, NJ, ${ }^{5}$ University of Iowa, Iowa City, IA

Background/Introduction: Incompatibility in Electronic Health Record (EHR) systems across hospital groups often hinders medical record exchange or shared management in postoperative spine patients necessitating emergent care at different hospitals. Given that patients undergoing complex lumbar spine procedures often require prompt multi-disciplinary palliative care, a lack of familiarity can plausibly pose hurdles to efficient care. However, limited or no information exists quantifying the association of postoperative fragmented spine care. In the current study, the authors investigate the association of 30-day post-discharge fragmentation with outcomes and resource utilization after lumbar spine surgery using a comprehensive, all-payer, readmission cohort.

Materials/Methods: The National Readmission Database 2013-2014 was queried to identify adult patients incurring 30-day readmission (via unplanned ED visit) following a lumbar spine procedure. Fragmentation in care was defined as patients readmitted to hospitals other than the index (original) center where the lumbar spine surgery was performed. Primary outcome measures were discharge disposition after readmission, complications and resource utilization (costs, length of readmission stay). Multivariable regression techniques fitted with generalized estimating equations and adjusted for confounders investigated the association of post-discharge fragmentation of care with primary outcome endpoints.

Results: Overall, 1269 patients incurred a 30-day readmission following lumbar spine procedure using ED services. Post-fragmentation in care was noted in $25.5 \%$ patients $(n=324)$. In multivariable regression models, prompt postoperative care at non-indexed hospitals for unplanned readmission within 30-days after lumbar surgery was associated with significantly lower propensity for wound complications (OR:0.32; $\mathrm{p}<0.001)$, infections (OR:0.35; $\mathrm{p}<0.001)$, and shorter readmission stay $(-0.9$ days, $\mathrm{p}=0.002)$ [Figure 1]. Interestingly, no differences were noted in discharge disposition (after readmission), neurological complications (OR: $0.64 ; \mathrm{p}=0.378)$, venous thromboembolism (OR: $1.28 ; \mathrm{p}=0.185)$ or resource utilization $(-\$ 882 ; \mathrm{p}=0.149)$ in patients incurring fragmented care versus those with continuity of care (readmitted to original/index hospital where lumbar surgery was performed). However, propensity of renal complications was relatively higher in patients with fragmented care (OR: $1.5 ; \mathrm{p}=0.04)$.

Discussion/Conclusion: Approximately, a quarter of patients undergoing lumbar spine surgery will experience post-discharge fragmentation. The study underscores the potential significance of prompt palliative care at non-indexed hospitals in optimizing outcomes including wound care, infection control and resource utilization after lumbar spine surgery. 


\section{Poster 22. Influence of Geometry and Architecture of 3D-printed Hyperelsastic Bone ${ }^{\circledR}$ Composite Scaffolds on Spinal Fusion in the Rat}

Mitchell Hallman, B.A. ${ }^{l}$, David Ellenbogen, B.A. ${ }^{2}$, Jonathan Yamaguchi, B.S. ${ }^{2}$, Allison Greene, B.S. ${ }^{3}$, Adam Jakus, PhD ${ }^{4}$, Vivek Shah, B.S. ${ }^{2}$, Ramille Shah, PhD ${ }^{4}$, Stuart Stock, PhD ${ }^{4}$, Wellington Hsu, M.D. ${ }^{5}$, Erin Hsu, $P h D^{6}$

${ }^{1}$, Raleigh, $\mathrm{NC},{ }^{2}$, Chicago, IL,${ }^{3}$, Chicago, Illinois, ${ }^{4}$ Northwestern University, Chicago, Illinois, ${ }^{5}$ Northwestern Memorial Hospital, Chicago, Illinois, ${ }^{6}$ Northwestern University Department of Orthopaedic Surgery, Chicago ,IL

Background/Introduction: We previously developed a recombinant growth factor-free 3D-printed Hyperelastic Bone ${ }^{\circledR}$ composite (HBC) composed of hydroxyapatite (HA) and demineralized bone matrix (DBM) for spinal fusion. Here, we aimed to identify the optimal geometry, pore size, and strut orientation to promote osseointegration, vascular infiltration, and spine fusion.

Materials/Methods: Inks containing HA and DBM particles in a poly(lactide-co-glycolide), PLG) elastomer were 3D-printed into scaffolds. Eighty-four female Sprague-Dawley rats underwent L4-L5 PLF with placement of one of seven HBC scaffold iterations $(n=12)$ with varying strut angle, macropore size, and strut alignment $\left(90^{\circ} / 500 \mu \mathrm{m}\right.$ aligned, $45^{\circ} / 500 \mu \mathrm{m}$ aligned, $90^{\circ} / 1000 \mu \mathrm{m}$ aligned, $45^{\circ} / 1000 \mu \mathrm{m}$ aligned, $90^{\circ} / 1000 \mu \mathrm{m}$ offset, $45^{\circ} / 1000 \mu \mathrm{m}$ offset, vs. no macropores). Fusion was assessed via manual palpation 12 weeks post-operatively. Vascularization and bone growth from the transverse processes into the scaffold macropores were quantified using microCT imaging.

Results: The $45^{\circ} / 1000 \mu \mathrm{m}$ scaffolds with aligned struts achieved the highest average fusion score $(1.61 / 2)$. Both the $45^{\circ} / 1000 \mu \mathrm{m}$ aligned and $90^{\circ} / 1000 \mu \mathrm{m}$ aligned scaffolds demonstrated fusion rates of $100 \%$, which was significantly greater than the $45^{\circ} / 500 \mu \mathrm{m}$ aligned iteration $(\mathrm{p}<0.05$, Fig. $1 \mathrm{~A})$, and the $45^{\circ} / 1000 \mu \mathrm{m}$ aligned scaffold had the highest osteointegration score. All porous scaffolds demonstrated vascularization through every macropore. Vessels were observed protruding through the bony fingers growing from the TPs into the macropores (Fig.1B-C).

Discussion/Conclusion: The HBC scaffold represents a novel biomaterial that demonstrates success in a clinically relevant rat model. When viewed independently, strut angles of $45^{\circ}$ and $90^{\circ}$ both allow for bone regeneration, and a pore size of $500 \mu \mathrm{m}$ or greater is sufficient to achieve fusion. However, aligned struts printed at $45^{\circ}$ with $1000 \mu \mathrm{m}$ macropores provide the optimal configuration to maximize osseointegration and fusion. That new blood vessels were found within every macropore further suggests that HBC scaffolds provide a conductive environment for bone regeneration and vascular infiltration. Further studies will directly compare the efficacy and mechanisms of action of the HBC with that of rhBMP-2 delivered on an absorbable collagen sponge (INFUSE ${ }^{\mathrm{TM}}$, the gold standard control for this model) in promoting spinal fusion. HBC scaffolds represent a novel, growth-factor-free, off-the-shelf biomaterial that has significant potential as a bone graft substitute for use in spinal surgery. 


\section{Poster 23. Revision rate following Baxano iO-Flex foraminal decompression}

John France, $M D^{l}$; Samuel Adams, $M D^{2}$

${ }^{l}$ West Virginia University, Morgantown, $W V,{ }^{2}$, morgantown, wv

Background/Introduction: Foraminal stenosis describes the encroachment hard and soft tissues on an exiting nerve root which commonly presents alongside common degenerative changes seen with aging. This may occur in conjunction with central canal stenosis or as a separate entity, in the absence of central stenosis. Commonly presenting symptoms are buttock and radicular leg pain on the affected side. Initial management involves non-operative modalities such as physical therapy and injections. If the patient has failed these conservative measures, operative management may be indicated. One recently developed technique is the iO-Flex system, which involves a less invasive approach to widen the neuroforamen in an anterior-posterior fashion. This approach allows minimal soft disruption of the surrounding tissues. Cadaveric studies have demonstrated the efficacy of this technique at increasing the dimensions of the foramen but limited clinical data is available. One study of 9 patients at 1 year did not find any reoperation rates and resulted in improvements in VAS, Zurich Claudication Questionnaire, and SF-36. A larger study involving 67 patients with average 3.5 month follow-up had similar outcomes. We have collected data on 37 subjects undergoing surgery between August 2013 and September 2017 with average 1 year follow-up (range 2 weeks-5 years).

Materials/Methods: We retrospectively reviewed data from a single surgeon at a level-one University hospital setting. A total of 37 subjects underwent the iO-Flex foraminal decompression in this time period. The primary outcome measure was re-operation. Chart review from our institution records was used for data recovery.

Results: Eight of 37 (22\%) subjects underwent revision surgery for unrelenting and recurrent symptoms at the same level at an average of 24.5 months. Revision surgery was most commonly (7/8) anterior lumbar interbody fusion.

Discussion/Conclusion: Less invasive techniques for foraminotomy may preserve soft tissues and can provide relief of foraminal stenosis. The iO-Flex system is one method of widening the neuroforamen with such a technique. Patients should be counseled on the risk of revision surgery for unrelenting or recurrent symptoms prior to such procedures as a relatively high number (22\%) go on to revision surgery within 24.5 months. 


\section{Poster 24. Lumbar spine stiffness and motion as a function of sagittal alignment under complex loading}

Jamie Baisden, $M D^{l}$, John Humm, $M S^{2}$, Frank Pintar, $P h D^{3}$, Narayan Yoganandan, $P h D^{l}$

${ }^{1}$ Medical College of Wisconsin, Milwaukee, Wisconsin, ${ }^{2}$ Medical College of Wisconsin, Milwaukee, WI, ${ }^{3}$, MIlwaukee, WI

Background/Introduction: Sagittal alignment and biomechanical loading are interrelated. Spine biomechanical studies, however, are limited to pure moment-type (follower load) moment-angulation responses. Aim: to determine the effects of spinal alignment and complex-combined loading on lumbar spine stiffness.

Materials/Methods: Seven male cadaveric (with minimal degenerative changes) lumbar spinal columns (T12-S1) $(57 \pm 10$ years, $171 \pm 10 \mathrm{~cm}, 68 \pm 19 \mathrm{~kg})$ were fixed at ends such that T12/L1 and L5/S1 joints were unconstrained. Six-axis loads cells at the ends were used to obtain force and moments. Specimens were fixated in a custom device to change the positioning of S1 relative to T12 to induce different sagittal alignment conditions. Positions ranged from natural lordosis to a straight spine (parallel L5 and L1 endplates). Two intermediate alignment conditions were also selected. Axial loading was applied at each position using an electro-hydraulic piston. Three non-collinear retroreflective targets were inserted into each anterior bodies at each level to measure the three-dimensional motions (kinematics).

Results: Alignment-based stiffness was computed using force-deflection time-histories. Stiffness increased from the natural to the straight alignment condition for all specimens (Figure-1: bar chart, x-rays). Motions were complex and their plots for each level and alignment will be presented.

Discussion/Conclusion: This is the first study to determine the role of spinal alignments and complex axial loading on lumbar columns. Stiffness and segmental motions were computed using this novel experimental technique. Although follower loads can be applied, fixed end-conditions were used for alignment as the first step. Increasing stiffness from normal lordotic to straightened posture results from increased loadsharing of vertebral bodies and discs. Increased motions from the straightened to natural postures suggest the role of ligaments and posterior joints/ facet complex in the load sharing. It is likely that majority of change in the axial spine stiffness of the column was caused by increasing compression of the L5/S1 disc as the posture transitioned to a straight sagittal alignment. The reduced sacral slope produces higher compression of the lumbosacral joint in a straightened spine resulting in increased stiffness. Changes in spinal alignment over time affect internal load sharing and may clinically alter the course of spinal degeneration. 


\section{Poster 25. Industry Payments to Orthopaedic Spine Surgeons Reported by the General Open Payments Database: 2014 to 2017}

Neil Pathak, $B S^{l}$, Michael Mercier, $B S^{l}$, Anoop Galivanche, B.S. ${ }^{l}$, Elbert Mets, BA ${ }^{l}$, Patawut Bovonratwet, $B S^{l}$, Arya Varthi, $M D^{2}$, Jonathan Grauer, $M D^{3}$

${ }^{1}$ Yale School of Medicine, New Haven, CT, ${ }^{2}$ Yale University, New Haven, CT, ${ }^{3}$, New Haven, CT

Background/Introduction: The Open Payments Database (OPD) was developed to optimize transparency related to physician-industry relationships, and has released four calendar years of data (2014 to 2017). To our knowledge, no prior studies have examined payments made to spine surgeons from the more recent years of this database. With increased attention being given to this topic, the current study aims to investigate trends in general (defined as nonresearch) payments reported in the OPD to orthopaedic spine surgeons from 2014 to 2017. As OPD does not distinguish sub-specialties within neurosurgery (i.e. neurosurgery spine surgery), the current study focused on orthopaedic spine surgeons.

Materials/Methods: General industry payments made to orthopaedic spine surgeons (as identified by OPD) from 2014 to 2017 were characterized by year with analysis of: number of surgeons receiving payments, median payment, payment sub-type, and physician breakdown by census region. Mann-Whitney $\mathrm{U}$ tests were used to compare median values.

Results: The number of orthopaedic spine surgeons receiving general payments increased from 2014 to 2017 (1539 to 1673). Later year median general payments per surgeon were compared to the median payment of 2014 ( $\$ 1051): 2015$ ( $\$ 1070: \mathrm{p}=0.375), 2016(\$ 1263: \mathrm{p}=0.012)$, and 2017 (\$978: $\mathrm{p}=0.561)$ (Figure 1). In 2014, the top $10 \%$ of compensated orthopaedic spine surgeons received $89 \%$ of the total payment to orthopaedic spine surgeons, top 5\% received 79\%, and the top $1 \%$ received $55 \%$. The median payment for these three strata remained stable over the four years evaluated ( $p>0.05$ ). From 2014 to 2017, the median payment for honoraria $(\mathrm{p}<0.001)$, education $(\mathrm{p}=0.025)$, and food/beverage $(\mathrm{p}<0.001)$ payments increased significantly, while the median payment for royalty/license $(\mathrm{p}=0.038)$ and grant $(\mathrm{p}=0.025)$ payments decreased significantly. Additionally, West census region orthopaedic spine surgeons earned the highest median payment per year with the exception of 2014 (Northeast).

Discussion/Conclusion: Many expected industry payments to surgeons to decrease over time under the public scrutiny of the OPD. The current study showed fluctuation in median general payment per orthopaedic spine surgeon, but no net change over the four years studied. While there is nothing inherently concerning about these findings, they are important to note in the current age of greater physician-industry transparency. 


\section{Poster 26. Midline Lumbar Fusion (MIDLF) Achieves Clinically Equivalent Segmental Lordosis Compared with "Open" Transforaminal Lumber Interbody Fusion}

Joseph Laratta, $M D^{l}$, Jamal Shillingford, $M D^{l}$, Karishma Gupta, $M P H^{2}$, Steven Glassman, $M D^{3}$, Charles Crawford, $M D^{4}$, Jeffrey Gum, $M D^{5}$, Mladen Djurasovic, $M D^{4}$

${ }^{I}$ The Spine Hospital at Columbia University, New York, $N Y,{ }^{2}$, Las Vegas, $N V,{ }^{3}$, Louisville , Kentucky, ${ }^{4}$, Louisville, KY , ${ }^{5}$ Norton Leatherman Spine Center, Louisville, $K Y$

Background/Introduction: The midline lumbar fusion (MIDLF) procedure has been developed as an alternative lumbar interbody fusion technique to improve recovery and minimize postoperative morbidity. The MIDLF procedure involves a midline approach, cortical bone trajectory (CBT) screws, and microsurgical laminectomy with a posterior interbody fusion. Unfortunately, the procedure's effect on segmental lordosis is not well characterized. The purpose of the current study was to compare the change in local sagittal parameters after MIDLF to traditional "open" transforaminal interbody fusion (TLIF) procedures.

Materials/Methods: A retrospective review of lumbar interbody fusions performed between 2013 and 2016 at a high-volume center by three senior spine surgeons was performed. Twenty traditional "open" TLIF patients were matched based on age, gender, diagnosis, and surgical level to twenty MIDLF patients. Anterior disc height, posterior disc height, and segmental lordosis were measured on preoperative and postoperative upright lumbar radiographs.

Results: All patients underwent a single-level procedure for degenerative spondylolisthesis. In each group, two (10\%) of the procedures were at the L3-4 level and 18 (90\%) were at the L4-5 level. The average age in the TLIF and MIDLF groups was $61.45+/-6.60$ years and $59.15+/-11.32$ years, respectively (p $=0.219)$. In the MIDLF group, the overall change in segmental lordosis, anterior disc height and posterior disc height was +0.46 degrees, $4.73 \mathrm{~mm}$, and $2.03 \mathrm{~mm}$, respectively. In the TLIF group, the overall change in segmental lordosis, anterior disc height and posterior disc height was +1.98 degrees, $4.59 \mathrm{~mm}$, and 1.50 $\mathrm{mm}$, respectively. The segmental change in lordosis was significantly greater in the TLIF group than the MIDLF group $(p=0.038)$, while the change in anterior and posterior disc heights was not statistically different ( $\mathrm{p}=0.455$ and $\mathrm{p}=0.237$, respectively).

Discussion/Conclusion: The MIDLF and open TLIF procedures achieve a clinically equivalent, albeit small, increase in segmental lordosis. Both procedures are effective at increasing anterior and posterior disc height and thus, provide indirect foraminal decompression. The effect of these radiographic findings on clinical outcome is yet to be determined. 
Poster 27. The Efficacy of Intraoperative Multimodal Monitoring in Pedicle Subtraction Osteotomies of the Lumbar Spine

Jianning Shao, B.A. ${ }^{l}$, Maxwell Lee, $B S^{l}$, Shreya Louis, $B A^{2}$, Konrad Knusel, $B A^{3}$, Bryan Lee, $M D^{2}$, Dominic Pelle, $M D^{2}$, Joseph Tanenbaum, $B S^{4}$, Jason Savage, M.D. ${ }^{5}$, Thomas Mroz, M.D. ${ }^{6}$, Michael Steinmetz, $M D^{7}$

${ }^{1}$, Cleveland, Ohio, ${ }^{2}$ Cleveland Clinic, Cleveland, $\mathrm{OH},{ }^{3}$ Case Western, Cleveland, $\mathrm{OH},{ }^{4}$ Case Western Reserve University School of Medicine, Cleveland, Ohio, ${ }^{5}$ The Cleveland Clinic, Cleveland, Ohio, ${ }^{6}$ Cleveland Clinic Foundation, Neurological Institute, Cleveland, $\mathrm{OH},{ }^{7}$ Cleveland Clinic, Department of Neurosurgery, Cleveland, $\mathrm{OH}$

Background/Introduction: Iatrogenic spine injury remains one of the most dreaded complications of PSOs and spine deformity surgeries. As such, IOM, which has the potential to provide real-time feedback regarding cord integrity, has become the gold-standard in such operations. However, while the benefits of IOM are well-established in PSOs of the thoracic spine, its utility in PSOs of the lumbar spine has not been robustly documented.

Materials/Methods: All adult patients who underwent lumbar PSOs at our institution from 2007 to 2017 were analyzed via retrospective chart review. Patients were categorized into one of two groups on the basis of IOM guidance. PDQ-39 quality of life (QOL) scores were gathered and multivariate analysis was performed for quantitative comparison of QOL scores. In addition, the proportion of patients who reached minimal clinically important difference (MCID),defined as an increase in PDQ-39 score of 4.72, was also determined, and statistical analysis was preformed to determine whether IOM had a significant impact on achieving MCID.

Results: A total of 101 patients were included in the final analysis. Multivariate analysis shows that parameters with a statistically significant impact on achieving MCID included age $(\mathrm{OR}=1.11, \mathrm{p}=0.001)$ and female sex $(\mathrm{OR}=7.6$, p-value $=0.005)$. Importantly, IOM usage was not correlated with a statistically significant increase in QOL scores $(p=0.230)$ or achievement of MCID 3 months after surgery $(p=0.196)$.

Discussion/Conclusion: In our particular cohort, IOM did not lead to statistically significant improvement in outcomes in patients undergoing PSOs of the lumbar spine ( $p=0.230)$. However, it is worthwhile to note that while statistical significance was no shown in this particular study, IOM is rapidly becoming the standard of care, and can be considered worthwhile if it prevents even one iatrogenic injury. The existing clinical equipoise, however, indicates future studies in this arena are necessary. 


\section{Poster 28. Is Dental Prophylaxis Required Following Spinal Fusion? A Systematic Review and Call for Evidence}

Parker Martin, Bachelor of Science, Bachelor of Art ${ }^{l}$, Rajbir Hundal, $\mathrm{MD}^{2}$; Ilyas Aleem, MD, MSc, FRCSC $^{3}$, Kathryn Matulich, MSN, ACNP-BC ${ }^{2}$

${ }^{1}$ University of Michigan Medical School, Ann Arbor, MI, ${ }^{2}$, Ann Arbor, MI, ${ }^{3}$ University of Michigan, Ann Arbor, MI

Background/Introduction: Controversy exists regarding the need for antimicrobial prophylaxis prior to dental procedures following spinal fusion. Neither spinal surgeons nor dentists have evidence-based guidelines regarding dental prophylaxis in protecting against hematogenous infection of spinal instrumentation. As the number of spinal fusions increases significantly, the lack of clear guidelines poses a problem as no standard of care has been established for these patients. In this review, we attempt to synthesize a comprehensive summary of the published literature to provide recommendations on the use of antimicrobial prophylaxis before dental procedures in patients with a history of spinal fusion.

Materials/Methods: We searched PubMed, Web of Science, Cochrane Library, and EMBASE databases from inception to February 2018. Eligible studies included patients with a history of spinal fusion treated with or without antimicrobial prophylaxis in preparation for dental procedures. Two reviewers independently assessed the eligibility of potential studies and extracted data. Outcomes of interest were the indications and efficacy of antimicrobial prophylaxis to protect against infection of spinal prostheses with dental origin.

Results: A total of 1909 articles were initially screened. After inclusion and exclusion criteria were applied, one study was found specifically relating to dental prophylaxis and spine surgery. This was an expert survey which revealed that approximately two-thirds of spine surgeons would not recommend antimicrobial prophylaxis for patients with a history of uncomplicated lumbar fusion. The survey, as well as objective studies and professional organization guidelines on dental prophylaxis in patients with total hip and knee replacements, were reviewed to add context to the controversy.

Discussion/Conclusion: There is a significant paucity of literature regarding dental prophylaxis in spine surgery patients. The continued disagreement revealed by expert surveys is likely due to the lack of research addressing this population in combination with shifting recommendations from professional organizations. Although there has been a recent movement away from recommending antimicrobial prophylaxis before dental work in patients with other forms of orthopaedic prostheses, the gap in the literature addressing spine patients represents an important question that requires more targeted and specific research. 


\title{
Poster 29. Incidental Extraspinal Imaging Findings on Adult EOS Whole Spine Radiographs: Preliminary Findings on Prevalence and Clinical Importance
}

\author{
Christopher Martin, $M D^{I}$, Takashi Takahashi, $M D^{2}$, Lilly Wood, $B S^{2}$, Joseph Luchsinger, $M D^{l}$ \\ ${ }^{l}$ University of Minnesota, Minneapolis, $M N,{ }^{2}$, Minneapolis, $M N$
}

Background/Introduction: Radiographic evaluation of adult spinal deformity frequently includes full length standing radiographs. These images include significant skeletal and soft tissue detail given that they include an image of the entire skeleton, as well as the thoracic and abdominal cavities. Thus, these images often capture incidental findings. The rate and clinical significance of incidental findings from spinal CT or MRI studies has been widely reported. However, the incidence and clinical significance of similar findings from whole spine radiographs has not been well established.

Materials/Methods: We retrospectively reviewed radiologists' dictations to identify the incidence of incidental extraspinal imaging findings on full length standing radiographs taken on an EOS machine at our institution between 10/2017 and 2/2018. The incidence and type of incidental finding was recorded, and we then conducted a chart review to identify the subsequent follow-up and clinical significance of the findings.

Results: 134 full body images were obtained during the study period. There were 125 incidental findings including 74 extraspinal arthritic/degenerative findings, 9 vascular findings, 4 patients with femoroacetabular impingement, and 9 pulmonary nodules. The overall clinical significance of the findings was low, and in none of the cases was any significant clinical follow-up recommended.

Discussion/Conclusion: In contrast to CT or MRI based imaging studies of the spine where clinically significant incidental extra-spinal imaging findings are not in-frequent, the incidence in this study of whole spine radiographs was low, although many benign extra-spinal findings may be identified. 


\section{Poster 30. Registry Outcomes of Cement Augmentation for Osteoporotic Vertebral Compression Fractures}

Paul Anderson, $M D^{l}$, Neal Shonnard, $M D^{2}$, Sigurd Berven, $M D^{3}$, Douglas Beall, M.D. ${ }^{4}$, Justine Norwitz, CEO $^{5}$, Derrick Wagoner, D.O. ${ }^{4}$, Sara Khor, MASc $^{6}$

${ }^{l}$ University of Wisconsin, Madison, WI, ${ }^{2}$, Tacoma, WA, ${ }^{3}$, San Francisco, CA, ${ }^{4}$ Clinical Radiology of Oklahoma, Edmond, OK, ${ }^{5}$ BenchMarket Medical, Seattle, WA, ${ }^{6}$ University of Washington Surgical Outcomes Research Center, Seattle, WA

Background/Introduction: Cement augmentation of painful osteoporotic vertebral compression fractures (VCF) has been shown to be beneficial and effective treatment. Data from clinical registries which record the safety and efficacy of vertebral augmentation has not been widely produced and the performance results of vertebral augmentation in a real-world application would be additive to existing literature and would serve to mitigate any controversy surrounding the procedure.

Materials/Methods: The Vertebral Compression Fracture (VCF) Registry included 732 patients with clinical and imaging confirmed symptomatic vertebral compression fractures who were then treated with vertebral augmentation. Mean age was 78.1 years (standard deviation of 10.5 years). Registry included 529 women (72\%) and 203 men (28\%). 616 (84\%) of patients had failed non-surgical management. Patients' reported pain was measured at baseline, 1,3, and 6 months post procedure using the Numeric Rating Scale (NRS) $0-10$. This was then asked again at the post procedure time points via a telephone survey.

Similarly, patient function was assessed through the Roland Morris (RM) standardized survey. The Roland Morris score ranges from $0-24$ with 0 being fully functional and 24 meaning function was severely impaired. Ten clinical sites participated submitting data which was visible for all participants via an online dashboard, updated as procedures were logged. Thoracic and lumbar vertebral compression fractures were treated with balloon-kyphoplasty or vertebroplasty. Post procedure CT exams were obtained and cement leakage was recorded if present.

Results: Median Numeric Rating Scale (NRS) measuring pain was 9 at baseline (interquartile range (IQR) 8-10), 2 at 1 month (IQR 0-5), 1 at 3 months (IQR 0-4), and 0 at 6 months (IQR 0-4). Roland Morris score ranging from $0-24$ measuring functionality was 21 at baseline (IQR 18-22), 12 at 1 month (IQR 6-16), 9 at 3 months (IQR 3-14), and 7 at 6 months (IQR 3-12).

Discussion/Conclusion: Real-world patient care outcomes show vertebral augmentation is effective in alleviating pain and improving function in patients with symptomatic vertebral compression fractures. Vertebral augmentation results in statistically significant improvements in pain and functionality post treatment at 1,3, and 6 months in typical patients presenting with painful VCFs. 


\section{Poster 31. Spine Surgery Patients have a Non-Responder Bias for who completes the HCAHPS surveys}

Rohil Malpani, B.S. ${ }^{l}$, Murillo Adrados, MD ${ }^{2}$, Michael Mercier, BS ${ }^{3}$, Ryan McLynn, B.S. ${ }^{l}$; Jonathan Grauer, $M D^{4}$

${ }^{1}$ Yale School of Medicine, New Haven, Connecticut, ${ }^{2}$ Yale School of Medicine, New Haven, CT,${ }^{3}$ Yale School of Medicine, New Haven, CT, ${ }^{4}$, New Haven, CT

Background/Introduction: The Hospital Consumer Assessment of Healthcare Providers and Systems (HCAHPS) survey is a publicly reported patient satisfaction survey. In addition to having the potential of impacting a hospital's reputation, it is now directly linked to government reimbursement. However, it is known that a minority of patients return this survey, and it is expected that there are non-response biases.

Materials/Methods: All adult inpatient spine surgery patients between January 2013-August 2017 at a single institution were selected for retrospective analysis. Patient demographics and perioperative outcomes were assessed as potential predictors of not returning HCAHPS surveys. Univariate and multivariate analyses were performed.

Results: Of 5,517 spine surgeries analyzed, 1,505 (27.28\%) patients returned the HCAHPS survey. The response rate was variable based on individual surveys (with statistically significant differences based on age, functional status, race, and ASA score) but not variable based on anatomic region of the spine surgery. Multivariate analysis revealed that patients who did not return the HCAHPS survey were more likely to be black/African American $(\mathrm{OR}=2.8, \mathrm{p}<0.001)$, have a higher ASA score (OR 1.76, $\mathrm{p}<0.001)$, and have had a major adverse event $(\mathrm{OR}=1.66 ; \mathrm{p}=0.001)$, minor adverse event $(\mathrm{OR}=2.50 ; \mathrm{p}<0.001)$, discharged to a destination other than home $(\mathrm{OR}=2.16, \mathrm{p}<0.001)$, hospital readmission $(\mathrm{OR}=2.58 ; \mathrm{p}<0.001)$, and a long hospital length of stay ( $>3$ days $)(\mathrm{OR}=1.28, \mathrm{p}=0.001)$.

Discussion/Conclusion: For spine surgery patients, patient characteristics and perioperative outcomes were found to be significantly associated with the nonresponse bias of HCAHPS surveys. Although the potential resultant bias in HCAHPS scores cannot be directly determined, this must be considered in interpreting the results of such satisfaction surveys given that less than one third of patients actually completed this survey in the study population. 


\section{Poster 32. Combined Magnetic Field Results in Higher Fusion Rates than Pulsed Electromagnetic Field Bone Stimulation after Thoracolumbar Fusion Surgery}

Barry Cheaney II, B.S. ${ }^{\text {, }}$ Mahboub Hashemi, M.D., MACS ${ }^{2}$, James Obayashi, B.S. ${ }^{\text {, }}$ Khoi Than, MD ${ }^{3}$ ${ }^{1}$ Oregon Health \& Science University, Portland, OR, ${ }^{2}$, Portland, OR, ${ }^{3}$ Oregon Health \& Science University, Portland, Oregon

Background/Introduction: Bone growth stimulators have been used as an adjunct to spinal fusion surgery in efforts to increase fusion rates. These electrical stimulators are designed to deliver electrical fields that modulate bone cell activity to enhance bone formation. In this study, fusion rates were compared in patients using pulsed electromagnetic field stimulation (PEMF; Orthofix, Lewisville, TX) versus combined magnetic field stimulation (CMF; DJO Global, Vista, CA) after thoracolumbar fusion surgery.

Materials/Methods: The authors retrospectively reviewed the medical records for patients who underwent thoracolumbar fusion surgeries (posterolateral only, interbody only, or posterolateral and interbody) by a single surgeon and were prescribed bone growth stimulators. The patients were separated into two groups, either PEMF or CMF, and computed tomography radiographic results at one year of follow-up were compared (solid fusion, stable nonunion, and pseudarthrosis). Data was analyzed with a Pearson's chi square test.

Results: A total of 46 patients were included; 19 were randomly prescribed PEMF and 27 were prescribed CMF. There were no significant differences between age, sex, BMI, or tobacco use in the two groups. The average number of spine levels treated was 4.63 for PEMF and 3.81 for CMF, with an average follow-up of 15.89 months and 14.70 months, respectively. In the PEMF group, 13/19 (68.4\%) demonstrated solid fusion, $1 / 19(5.3 \%)$ stable nonunion, and 5/19 (26.3\%) pseudarthrosis. In the CMF group, 21/27 (77.8\%) demonstrated solid fusion, $5 / 27(18.5 \%)$ stable nonunion, and 1/27 (3.7\%) psudarthrosis. These date are statistically significant $(\mathrm{p}=0.024)$. Two patients with PEMF required re-operation for pseudarthrosis, compared to zero patients with CMF.

Discussion/Conclusion: This is the first study to compare PEMF and CMF bone growth stimulators in patients who underwent thoracolumbar spinal fusions. Patients using CMF postoperatively appear to have higher rates of solid fusion, lower rates of pseudarthrosis, and lower rates of re-operation when compared to PEMF. 


\section{Poster 33. Posterior Thoracolumbar Instrumented Fusion for Burst Fractures: A Meta Analysis}

Felipe Ituarte, $M D^{l}$, Christina Goldstein, $M D^{l}$, Theodore Choma, $M D^{l}$, ALI NOURBAKHSH, $M D^{2}$

${ }^{1}$ University of MO Dept. of Orthopaedic Surgery, Columbia, MO, ${ }^{2}$ University of Missouri Dept. of Orthopaedic Surgery, Columbia, MO

Background/Introduction: The study was performed to elucidate if the current body of literature supports one posterior spinal fusion fixation method as objectively superior compared to another, by minimizing the rates of implant failure and progression of post-traumatic kyphosis.

Materials/Methods: An extensive electronic search was conducted using PubMed. Articles were examined against the inclusion and exclusion criteria. Data pertaining to kyphosis angle, Frankel score, vertebral level, blood loss, operation time, hospital stay, post-operative bracing, instrument failure, complications, and follow-up was collected. A random-effects model was chosen due to variation among the individual studies' patient populations and surgical methods. Posterior instrumentation constructs were categorized into tiers with permutations of 1 or 2 screws above and/or below, as well as inclusion/exclusion of an intermediate screw at the fractured level. The mean change in Kyphosis Angle (KA) correction associated with each tier was calculated for immediate postop and final follow-up. A moderator analysis was performed to determine the impact of the intermediate screw on the postop kyphosis angle.

Results: 23 publications were deemed eligible and included. The tier with two screws above and one below with an intermediate screw had the greatest initial change in KA postop and was best at maintaining the restored KA along with having the lowest implant failure at final follow-up $(\mathrm{P}<0.001)$, this was followed by two screws above and two below with an intermediate screw. The moderator analysis, treating the intermediate screw as a moderating variable, identified that initial postop restoration of KA change was similar in tiers with intermediate screw compared to tiers without one. However, at final follow-up, tiers with an intermediate screw were better able to maintain the mean change in KA compared to tiers without an intermediate screw $(\mathrm{P}<0.001)$. There was no difference between the periods of hospital stay $(\mathrm{P}=0.788)$ and blood loss $(\mathrm{P}=0.154)$ among different tiers.

Discussion/Conclusion: A fixation method consisting of two screws above and one below with an intermediate screw for thoracolumbar burst fractures showed the highest maintenance of sagital balance with the smallest loss in restored postoperative kyphosis angle and lowest implant failure at final follow-up. 
Poster 34. Endoscopic vs. Open Decompression for Lumbar Spinal Stenosis: an International, MultiCenter Analysis

Ryan Chiu, B.S. ${ }^{l}$, Saavan Patel, B.S. ${ }^{l}$, Amy Zhu, B.S. ${ }^{l}$, Ankit Mehta, $M D^{2}$

${ }^{I}$ University of Illinois at Chicago, Chicago, IL ${ }^{2}$, Chicago, IL

Background/Introduction: In patients over the age of 65, lumbar spinal stenosis (LSS) is a common cause of radicular and generalized back pain, and is currently the most common indication for back surgery. Endoscopic decompression offers a minimally invasive alternative to traditional, open laminectomy. However, comparison of these two surgical techniques has been largely limited to small, single-center studies. In this study, we perform the first international, multi-institutional comparison of endoscopic and open lumbar laminectomy in assessing surgical complications, readmissions, and reoperations.

Materials/Methods: The 2017 American College of Surgeons' National Surgical Quality Improvement Program (ACS-NSQIP) database, containing data from over 650 hospitals internationally, was queried to evaluate the effect of endoscopic guidance on adverse events. Propensity score matching was then utilized to adjust for potential confounders among baseline demographics, comorbidities, and diagnoses. Operative time, length of stay, readmission and reoperation rates, as well as the incidence of peri- and post-operative complications, were compared between endoscopic and open groups.

Results: A total of 10,726 single-level lumbar decompression patients were identified and included in this study, $35(0.33 \%)$ of whom were operated upon endoscopically. Aside from $2(5.71 \%)$ readmissions, including 1 unplanned readmission, there were no reported surgical complications within the endoscopic group. The mean length of stay for these patients was $0.86 \pm 1.44$ days, with procedures lasting an average of $91.89 \pm 46.72$ minutes. However, these endpoints did not differ significantly from those of the open group.

Discussion/Conclusion: Endoscopically-guided approaches to single-level decompression of the lumbar spine did not reduce the incidence of peri- or post-operative adverse events. This may be due to the already small incision lengths associated with single-level open procedures, particularly with the widespread adoption of microscopic surgery. The mortality rate associated with endoscopic decompression in our study was similar to that reported in the literature. However, in contrast with similar studies, our results demonstrated no significant reduction in surgical site infections, length of stay or operative time. These discrepancies support the need for larger and prospective studies on this topic. 
Poster 35. Using Hounsfield units to quantify optimal fixation techniques in spine surgery: A biomechanical study correlating AP-DEXA, Lateral-DEXA and CT to pullout strength

\author{
Wayne Cheng, $M D^{l}$, Olumide Danisa, $M D^{2}$; Qasim Zaidi, $M D^{3}$ \\ ${ }^{1}$, Colton, CA, ${ }^{2}$ Loma Linda University, Department of Orthopaedic Surgery, Loma Linda, CA, ${ }^{3}$ New \\ England Baptist Hospital, BOSTON, MA
}

Background/Introduction: The increasing prevalence of spinal instrumentation in patients with decreased bone mineral density (BMD) necessitates a modality that will predict spine construct integrity accurately and cost effectively. Antero-posterior dual-energy X-ray absorptiometry (AP-DEXA) scan is a costeffective method of assessing BMD. In patients with degenerative disease and/or vascular calcification, Tscores may be spuriously elevated despite low real bone mineral density. Osteoporosis disproportionately affects trabecular bone therefore assessing BMD from lateral-projection DEXA may provide a more accurate assessment as the trabeculae rich vertebral body is unobstructed by posterior elements in the lateral position. Hounsfield unit (HU) measurement taken from computed tomography (CT) is a user friendly and widely accessible technique for assessing bone quality. The aim of this study is to assess BMD from AP and Lateral-DEXA and to measure HU values from CT-scan, and to correlate these values with pedicle screw pullout and fatigue strength.

Materials/Methods: Six fresh-frozen human cadaver spines of average age 65 years old, and average Tscore -1.36, underwent AP and Lateral-DEXA scan followed by helical-CT scanning. 17 vertebrae were instrumented with pedicle screws and maximal tensile pullout strength was recorded. Fatigue testing was performed and maximal relative rotational and translational displacement was recorded. These biomechanical parameters were compared to AP and Lateral-BMD and HU Values.

Results: The correlation between $\mathrm{HU}$ value and pullout strength $(\mathrm{R} 2=0.776)$ was statistically significant. The correlation between AP-DEXA BMD and pullout strength $(\mathrm{R} 2=0.696)$ was also statistically significant. The correlation between Lateral-DEXA BMD and pullout strength $(\mathrm{R} 2=0.401)$ was statistically significant but poor. HU Value correlated with rotation and translation $(\mathrm{R} 2=0.54$ and $\mathrm{R} 2=0.43)$. AP-DEXA BMD correlated with rotation only $(\mathrm{R} 2=0.72)$. Lateral-DEXA did not correlate significantly with rotation or translation.

Discussion/Conclusion: HU measurement exhibits a statistically significant correlation with all biomechanical parameters measured (pullout strength, angular rotation and relative translation). HU measurements from CT-scan provide a high quality assessment of pedicle screw biomechanics. CT-scan may provide an incremental benefit in bone quality assessment over DEXA, however, this should be weighed against the disadvantages of cost and radiation. In contrast, opportunistic HU measurements taken from previously acquired CT-scans offer an assessment of bone quality at no additional cost. 
RF 01. Injectable Peptide Amphiphile Nanofiber Scaffolds Drastically Potentiate BMP-2-mediated Spine Fusion in a Rabbit Model

Allison Greene, B.S. ${ }^{l}$, Jonathan Yamaguchi, B.S. ${ }^{2}$, David Ellenbogen, B.A. ${ }^{2}$, Mitchell Hallman, B.A. ${ }^{3}$, Vivek Shah, B.S. ${ }^{2}$, Mark McClendon, PhD ${ }^{4}$, Samuel Stupp, PhD ${ }^{5}$, Stuart Stock, PhD ${ }^{6}$, Erin Hsu, PhD ${ }^{7}$, Wellington Hsu, M.D. ${ }^{8}$

${ }^{l}$, Chicago, Illinois, ${ }^{2}$, Chicago, IL,${ }^{3}$, Raleigh, $N C,{ }^{4}$ Northwestern University Department of Materials Science and Engineering, Chicago, IL, ${ }^{5}$ Northwestern University Department of Materials Science and Engineering, Chicago, IL, ${ }^{6}$ Northwestern University, Chicago, Illinois, ${ }^{7}$ Northwestern University Department of Orthopaedic Surgery, Chicago ,IL, ${ }^{8}$ Northwestern Memorial Hospital, Chicago, Illinois

Background/Introduction: : Infuse ${ }^{\mathrm{TM}}$ has been shown to produce high rates of spinal fusion in humans; however, the supraphysiologic doses of recombinant human bone morphogenic protein-2 (rhBMP-2) required can lead to serious complications. Previously, we developed peptide amphiphile nanofibers containing a BMP-2 binding motif (bPA) which reduce the required dose of rhBMP-2 needed to achieve fusion and prevent its burst release. In this study, we evaluated the efficacy of bPA to influence spinal fusion with sub-therapeutic doses of rhBMP-2 in a rabbit posterolateral fusion (PLF) model.

Materials/Methods: 86 New Zealand white rabbits underwent bilateral PLF at L5-L6 with 30, 15, or $5 \mu \mathrm{g}$ rhBMP-2/implant (i.e., 60, 30, or 10 rhBMP-2/animal) delivered via 1) Infuse ${ }^{\mathrm{TM}}$ (rhBMP-2/ACS), 2) bPA/ACS , 3) injectable bPA (bPA + collagen particles), or 4) injectable non-binding PA. Fusion was assessed by blinded manual palpation, where each fusion site was assigned a score of 0 (unfused, no bridging bone) or 1 (fused, L4-L5 bridging). New bone volumes were quantified using microCT imaging and Amira $^{\mathrm{TM}}$ software.

Results: All PA-based implants elicited significantly greater fusion rates and scores than Infuse ${ }^{\mathrm{TM}}$ alone (p $<0.05$ for all comparisons; Figure 1B-C). When loaded with $5 \mu \mathrm{g}$ rhBMP-2, the injectable bPA elicited a significantly greater fusion rate than both bPA/ACS and the injectable non-binding PA (90\% vs. $36 \%$ and $40 \%$, respectively; Figure 1C). Additionally, the injectable bPA implants generated significantly greater new bone volumes than bPA/ACS at $5 \mu \mathrm{g}$ rhBMP-2 $(\mathrm{p}<0.05$, Figure $1 \mathrm{~B})$.

Discussion/Conclusion: These data suggest that bPA, regardless of delivery vehicle, is a better carrier for BMP-2 than equivalently loaded Infuse ${ }^{\mathrm{TM}}$ (ACS) at sub-therapeutic doses of growth factor. While all bPA formulations elicited significantly higher fusion rates with reduced rhBMP-2 relative to Infuse ${ }^{\mathrm{TM}}$, the injectable bPA demonstrates the greatest efficacy at the lowest dose of growth factor, suggesting that this novel preparation is the superior vehicle for BMP-2 localization and release. The success of bPA in this large animal model, and its potential to reduce the number of side effects associated with BMP-2, make it a material of choice for pursuit in the clinical setting. 


\section{RF 02. Is there a correlation between Pfirrmann Grade and BMP-Signaling in Degenerative Disc Disease?}

Ayodeji Jubril, $B S^{l}$, Addisu Mesfin, $M D^{2}$, Avionna Baldwin, $B S^{3}$, Alex Hollenberg, $B S^{4}$, Roman Eliseev, $p h D^{3}$

${ }^{1}$, Rochester, $\mathrm{NY},{ }^{2}$ University of Rochester, Rochester, $\mathrm{NY},{ }^{3}$, rochester, $\mathrm{Ny},{ }^{4}$, rochester, ny

Background/Introduction: Degenerative disc disease (DDD) is a major burden to society. An estimated $80 \%$ of the population will be affected with back and neck pain. Bone morphogenetic proteins (BMPs) are known osteoinductors. To date no group has evaluated the role of BMP receptor Type I or ALK3 overexpression in the BMP-SMAD signaling pathway within the intervertebral disc as a therapeutic strategy for DDD. Pfirrmann grade is a validated MRI classification for degenerative disc disease.

Materials/Methods: We used degenerative disc specimen $(\mathrm{n}=37)$ from patients undergoing cervical and lumbar spine surgery. We also used control disc specimen $(n=13)$ from trauma patients. We rated the discs from Grade III to V. We evaluated expression of BMP/pSMAD/ALK3 in the degenerated and normal discs via immunohistochemistry. A Mann Whitney $U$ test was used for the comparison of quantitative data parameters between groups $(\mathrm{p}<.05)$.

Results: 37 patients were managed during the study period. Discs were stained for phos-SMAD, BMP, and ALK 3 then quantified by cell counts. The degenerative group resulted in averages of $14.8 \%, 13.1 \%$, and $8.1 \%$ for pSMAD, BMP, and ALK3 respectively. The average pfirrmann grade was 3.7.t test was used to compare expression levels of BMP, pSMAD, and ALK3 in degenerative discs and controls $(\mathrm{p}<.05)$. BMP and pSMAD resulted in significant differences, however ALK3 showed no significant difference $(\mathrm{p}=0.79)$. The Mann Whitney U test showed a significant relationship between the levels of BMP and PSMAD and Pffirmann grade $(\mathrm{p}=<.001)$. However, the expression of ALK3 was nonsignificant ( $\mathrm{p}=.107)$ Although there was an increasing trend in expression of BMP, pSMAD, and ALK3 in respect to grade, no statistically significant differences were appreciated by $\mathrm{t}$ test $(\mathrm{p}<.05)$.

Discussion/Conclusion: We have demonstrated BMP-2 and pSMAD are increased in patients with DDD. ALK3 levels remained the same and yielded no significant changes. This BMP-2 and pSMAD 1/5/8 upregulation suggests BMP/SMAD signaling is activated in the DDD process but perhaps not through ALK-3 binding. We conclude that there is a significant correlation between the expression levels of anabolic proteins BMP/ pSMAD, and degree of degradation in MRI grading based on the Pffirmann classification. 
RF 03. Compensatory mechanisms and clinical significance in lumbosacral isthmic spondylolisthesis with or without segmental kyphosis

Qingshuang Zhou, M.M ${ }^{1}$; Xu Sun, M.D. ${ }^{2}$, Zezhang Zhu, M.D. ${ }^{3}$, Bangping Qian, M.D. ${ }^{3}$, Zhen Liu, M.D. ${ }^{4}$, Yong Qiu, M.D. ${ }^{5}$, Bin Wang, $M B^{2}$

${ }^{l}$ Drum Tower Hospital Clinical College of Nanjing Medical University, Nanjing, Jiangsu, ${ }^{2}$ Spine Surgery, Drum Tower Hospital, Nanjing University Medical School,, Nanjing, Jiangsu, ${ }^{3}$, Nanjing, Jiangsu, ${ }^{4}$, Nanjing, Jiangse, ${ }^{5}$ Spine Surgery, Drum Tower Hospital, Nanjing University Medical School, Nanjing, Jiangsu

Background/Introduction: Anomalous sacropelvic morphology can result in an abnormal sacropelvic orientation and a disturbed global sagittal balance of the spine. Preserved disc height and presence of segmental kyphosis were risk factors of pseudoarthrosis or instrument breakage in isthmic spondylolisthesis (IS). The purposes of this study are to investigate characteristics of kyphotic configuration and to explore the sagittal alignment of kyphotic and non-kyphotic configuration in lumbosacral IS.

Materials/Methods: We retrospectively reviewed 271 patients with symptomatic single level IS who underwent surgical intervention at our center between January 2009 and April 2018. Based on the angulation profile of the olisthesis level, patients were divided into the kyphotic group and the nonkyphotic group. Slip parameters, pelvic parameters and sagittal parameters were measured commonly, and disc height of L5/S1 level, L5 incidence and L4S1 segmental lordosis was also measured for description characteristic of lumboscral portion. Pelvic incidence- lumbar lordosis (PI-LL) mismatch and the ratios of L4S1 segmental lordosis to lumbar lordosis (L4S 1/LL), and pelvic tilt to PI (PT/PI) were also calculated to evaluate the relationship between spinopelvic parameters and a morphologic parameter characteristic of each individual. Degeneration of each lumbar intervertebral disc was stimulated on MRI by the modified Pfirrmann standard.

Results: 26 (9.6\%) patients were in the kyphotic group and 215 patients in the non-kyphotic group $(\mathrm{P}<0.05)$. IS combined with prominently intradiscal kyphotic angulation were characterized by increased wedged of L5 vertebral bodies (anterior aspect larger than posterior), collapse of anterior and/or posterior disc space and more listhesis. Kyphotic IS demonstrated greater PI, PT, PT/PI, L5 incidence, PI-LL, but less L4S1 segmental lordosis and L4S1/LL than non-kyphotic IS $(\mathrm{P}<0.05)$. Moreover, severer degeneration of L5/S1 disc of kyphotic IS was evaluated on T2-weighted MRI through Pfirrmann classification. Patients with kyphotic IS were characterized by poor quality of life $(\mathrm{P}<0.05)$.

Discussion/Conclusion: 26 (9.6\%) patients occurred kyphotic angulation at olisthesis level, combined with high slip percentage and sever degenerative disc. Kyphotic IS was characterized with sagittal malignment, including insufficient LL, L4S1 segmental lordosis and pelvic retroversion, which resulted in a negative effect on patient quality of life. 
RF 05. What is the Cost of Pathology Testing of Retrieved Disc Material?

Casey Davidson, $M D^{1}$, Todd Chapman, $J r ., M^{2}$, Bruce Darden, $M D^{3}$, Christian Klare, $\mathrm{MD}^{4}$, Eric Laxer, $M D^{5}$, Susan Odum, PhD ${ }^{6}$, Alfred Rhyne, $M D^{7}$, P. Brad Segebarth, MD ${ }^{3}$, Leo Spector, MD ${ }^{3}$, Alden Milam, $M D^{5}$

${ }^{l}$ OrthoCarolina Spine Center, Charlotte, $N C,{ }^{2}$ Washington University School of Medicine, St. Louis, $\mathrm{MO},{ }^{3}$ OrthoCarolina, Charlotte, $\mathrm{NC},{ }^{4}$, Lebanon, $\mathrm{NH},{ }^{5}$, Charlotte, $\mathrm{NC},{ }^{6}$ OrthoCarolina Research Institute, Charlotte, $\mathrm{NC},{ }^{7}$ OrthoCarolina, Charlotte, North Carolina

Background/Introduction: During a discectomy procedure, material is removed and sent to the pathology lab for assessment to rule out disease. Pathology charges a fee and generates a pathology report which is sent to the surgeon. The surgeon typically receives the results after the surgery is over, which does not allow the surgeon to change treatment plan based on a positive result. Based on the Center for Medicare and Medicaid Services (CMS) fee schedule, the pathology fee totals $\$ 66.97$ for each discectomy procedure, which includes a $\$ 28.07$ technical fee of and a $\$ 38.9$ professional fee. In a value-based healthcare environment, cost containment is paramount. The purpose of this study was to quantify the economic burden of pathology assessments of retrieved disk material.

Materials/Methods: Pathology reports for 219 procedures at our institution from August 2016 through July 2018 were reviewed to determine the number of necessary pathology assessments, defined as a positive result warranting change in the standard workup and surgery. Additionally, using the methodology of Sherman et al (2010), the number of discectomy procedures performed in the U.S. in 2018 was estimated to be 500,741 . To calculate the local and national economic burden, the pathology fee was multiplied by the number of procedures.

Results: Of the 219 patients, there were no positive pathology results. At our institution, that is approximately a $\$ 14,666$ opportunity cost. At the national level, we estimated that 500,741 discectomy procedures were performed in 2018. Multiplying 500,741 procedures by $\$ 66.97$, totals $\$ 33,534,611$. This calculation assumes that material from every discectomy is sent to pathology. If we assume that $30 \%$ of the procedures do not include pathology testing, the cost would be approximately $\$ 24$ million.

Discussion/Conclusion: With continual pressures to contain cost and improve value, it is important for spine surgeons, and all health care providers, to lead the efforts. Routine pathology assessments of material removed during discectomy procedures represents an opportunity cost to institutions and national healthcare system. By eliminating this assessment, we could realize a savings to the US healthcare system of up to approximately $\$ 34$ million every year. 


\section{RF 06. Redefining Comorbidities: Do Concomitant Musculoskeletal Conditions Influence PROMIS Physical Function Scores?}

Nicholas Spina, $M D^{I}$, Ashley Neese, B.S. ${ }^{2}$, Nora Fino, $M S^{3}$, Angela Presson, PhD ${ }^{2}$, Natasha Greene, B.A. ${ }^{l}$, Chase Grover, $B S^{I}$, W. Ryan Spiker, $M D^{2}$, Brandon Lawrence, $M D^{2}$, Darrel Brodke, $M D^{2}$

${ }^{l}$ University of Utah, Salt Lake City, Utah, ${ }^{2}$, Salt Lake City, Utah, ${ }^{3}$ University of Utah, Salt Lake City, UT

Background/Introduction: Patient reported outcomes measures (PROs) are increasingly utilized to understand disease severity and efficacy of treatment. Legacy measures, such as the ODI, are diseasespecific and questions center around a specific pathology or joint. PROMIS Physical Function (PF), an emerging PRO, may be administered across musculoskeletal systems and addresses a domain of health rather than a specific disease state. Little is known regarding how concomitant musculoskeletal pathology influences PROMIS PF scores.

Materials/Methods: A retrospective review of orthopaedic spine patients presenting to a tertiary referral center between 2013-17 was performed. Patients were included who completed at least 1 PROMIS PF computer adaptive testing questionnaire. All patients were additionally asked, "Are your activities significantly limited by another body part that you are not being seen for today?" Baseline demographics were recorded including age, BMI, gender, Charlson Comorbidity Index, and smoking status. For patients with >1 PF CAT score within 12 months, the rate of conversion from no to yes, yes to no, or consistency in answer was calculated. Statistical analysis was performed to compare "yes" and "no" cohorts in terms of PROMIS PF CAT scores and demographic information.

Results: 1188 unique spine patients were identified with a total of 6016 PF CAT observations. 638 (54\%) of patients answered "yes" to having their physical function affected by another body part. The "Yes" cohort had a lower mean PF CAT (34.6 vs 37.1), were of equal age (64.2 vs 63.2), had higher CCI scores (2.6 vs 1.9), and no difference in BMI. $11.6 \%$ of patients converted from no to yes and $11.6 \%$ converted from yes to no within a 12 month interval. The mean difference in PF CAT scores in converting from "no" to "yes" was 0.3. Univariable and multivariable analysis identified PROMIS PF and CCI scores as significantly associated with answering "yes" (OR $0.98 \mathrm{p}=<.001$, OR $1.11 \mathrm{p}=<.001)$.

Discussion/Conclusion: Patients with orthopaedic comorbidities are exceedingly common in the spine population and present with lower PF CAT scores. Providers, using PROMIS PF scores, must account for the effect of other disease processes when treating spinal pathology. Further investigation is required to understand comorbidity effects following specific intervention. 
RF 07. A Retrospective Study of Postoperative Opioid Prescribing Practices in Decompressive Lumbar Spine Surgery Prior to and Following 2017 Ohio Legislative Reforms

Robert Winkelman, $B S^{l}$, Michael Kavanagh, $B B A^{2}$, Joseph Tanenbaum, $B S^{3}$, Dominic Pelle, $M D^{4}$, Thomas Mroz, M.D. ${ }^{5}$, Michael Steinmetz, $M D^{6}$

${ }^{I}$ Carnegie Mellon University, Cleveland Heights, Ohio, ${ }^{2}$ Case Western Reserve University School of Medicine, Cleveland Heights, Ohio, ${ }^{3}$ Case Western Reserve University School of Medicine, Cleveland, Ohio, ${ }^{4}$ Cleveland Clinic, Cleveland, $\mathrm{OH},{ }^{5}$ Cleveland Clinic Foundation, Neurological Institute, Cleveland, $\mathrm{OH},{ }^{6}$ Cleveland Clinic, Department of Neurosurgery, Cleveland, $\mathrm{OH}$

Background/Introduction: In an effort to combat the ongoing opioid epidemic, on August 31st, 2017, the state of Ohio enacted legislation limiting the dosage and duration of opioid prescriptions. According to the law, initial opioid prescriptions written for the treatment of acute pain may not exceed seven days duration nor exceed an average of 30 morphine equivalent doses (MEDs) per day. As opioids are a mainstay in the management of acute post-operative pain following spine surgery, we sought to evaluate the effect of this reform on prescribing practices at our institution.

Materials/Methods: This study is a retrospective review of patients from a single healthcare system who underwent decompression surgery of the lumbar spine between September 1st, 2016 - August 31st, 2018. Patients were identified using Current Procedural Terminology codes $63047+/-63048$. Postoperative opioid medication orders were obtained from the electronic health record system. Using these data, a comparison of 90-day post-discharge opioid prescriptions was performed between patients who underwent surgery in the year preceding (September 1st, 2016 - August 31st, 2017) and the year following (September 1st, 2017 - August 31st, 2018) the legislation's implementation.

Results: Nine hundred and eighty-six patients underwent isolated lumbar decompressive surgery during the two years evaluated. Patients undergoing decompression surgery in the year following reform were found to have received, on average, 196 fewer MEDs (95\% CI:-304.4 to -89.1) over the 90 days following discharge compared to patients who had surgery in the year prior. Additionally, patients who had surgery in the year following the reform were observed to have 2.8 fewer days (95\% CI:-4.5 to -1.0) of total opioids supplied, on average, than patients in the year prior (Figure 1). No difference was noticed in the overall number of prescriptions between years (0.1 prescriptions, $95 \%$ CI:-0.3 to 0.1$)$.

Discussion/Conclusion: At our institution, patients undergoing decompression surgery were prescribed significantly fewer opioids in the 90 days following spine surgery in the year after new restrictions were placed on opioid prescriptions compared to patients who received surgery in the year prior. Further research into how this decrease may have impacted patient reported outcomes following surgery is ongoing. 


\section{RF 08. Opioid Prescription Patterns among United States Spine Surgeons Compared To Other Orthopaedic Subspecialties}

Justin Koh, $M D^{1}$, Chadi Tannoury, $M D^{2}$

${ }^{l}$ Boston Medical Center, Boston, MA, ${ }^{2}$ Boston University Medical Center, Boston, MA

Background/Introduction: The US opioid epidemic has received particular attention with 33091 reported deaths from narcotic overdose in 2015. A recent survey comparing US and Japanese opioid prescribing patterns from the AOA-JOA traveling fellows included 76 US orthopedic surgeons representing 8 subspecialties. We used this data to compare opioid prescribing patterns among US spine surgeons to other orthopaedic subspecialties.

Materials/Methods: The survey included data based on surgeon demographics(gender, age, years in practice), patient baseline opioid use(pre/post-operative opioid use, types of opioid agents, duration of opioid use), perioperative pain management (procedure/institution factors, multimodal analgesic therapy), and responses to questions regarding opioid use attitudes. Survey responses were recorded as either categorical or semi-continuous variables. Statistical analysis was performed using chi-squared analysis for categorical responses and Kolmogorov-Smirnov testing to a statistical significance level of $p<0.05$. Parameters that trended toward significance to $\mathrm{p}<0.10$ were also recorded. Statistical analysis was performed using Stata statistical analysis software [StataCorp, College Station, Texas].

Results: Spine patients were more likely using opioid medications prior to evaluation by spine surgeons compared to non-spine patients $(\mathrm{p}=0.029)$. Patients with spine pathologies treated non-operatively were also more likely to be prescribed opioids $(\mathrm{p}=0.006)$. Post-operatively, spine patients were less likely to stop opioid prescriptions on the intended time $(\mathrm{p}=0.046)$. Peri-operatively, spine patients were more likely to be given muscle relaxant medications, and less likely to receive local/regional anesthesia $(\mathrm{p}=0.0025)$.

Additionally, spine patients showed a trend toward association for requesting additional opioids beyond their initial prescription( $\mathrm{p}=0.057)$. Spine surgeons showed a trend toward association for choice of opioid agent prescribed-spine patients were less likely to receive codeine/tramadol analogs $(\mathrm{p}=0.062)$. When surveyed on opinions regarding opioid use, US spine surgeons did not show significantly different opinions regarding opioid use compared to surgeons in other subspecialties.

Discussion/Conclusion: Spine patients did demonstrate statistically significant patterns of opioid use, particularly regarding opioid use prior to evaluation by orthopedic surgeon and use of opioids for nonoperative pathologies. They were also less likely to stop opioid prescriptions on time. Surgeon prescription patterns and perceptions of opioid use were not significantly different for spine surgeons compared to other subspecialties. 


\section{RF 09. Surgeon Preference For Radiologist Interpretation of Deformity Radiographs - A Survey of LSRS Membership}

Christopher Martin, $M D^{l}$, David Polly, Jr, $M D^{l}$, Takashi Takahashi, $M D^{2}$

${ }^{1}$ University of Minnesota, Minneapolis, $M N,{ }^{2}$, Minneapolis, $M N$

Background/Introduction: The radiologist interpretation of scoliosis films is non-standardized, with some practitioners providing detailed measurements of the deformity whereas others defer the interpretation of the deformity to the ordering surgeon. For radiologists, the standard of care is not clear, and this creates confusion in terms of how much interpretation is required. However, detailed radiologist reports sometimes conflict with the surgeon's interpretation, which can create confusion for patients who receive the reports, or in extreme cases can lead to insurance denials. Thus, the purpose of this study was to help establish a standard for interpretation of these films by surveying the ordering surgeons and documenting expert opinion about the amount and type of radiologist interpretation that is requested.

Materials/Methods: We designed a survey-monkey survey which aimed to look at standard practice for radiologist dictation of scoliosis radiographs. 12 questions were sent to LSRS membership via email with a description of the study. One follow-up email was also sent to non-responders.

Results: The rate of completed surveys was 46 out of 185 LSRS members (25\%). 37 respondents (80\%) work in academic institutions. 33 were orthopaedists (71\%), and 13 were neurosurgeons (28\%). 50\% reported that radiologists level of detail in dictations was inconsistent at their institution. Detailed numeric reporting was rare $(6.5 \%)$. When the radiologist did provide numeric measurements, surgeons reported that they often differed from the surgeon's own measurements, with only 2 respondents (4.5\%) reporting that the measurements "rarely" differed from the surgeon's. 39\% reported that radiologist measurements which differed from the surgeon's had led to insurance denials for their patients. The majority of respondents (70\%) did not want the radiologist to provide detailed numeric measurements of the deformity. $91 \%$ reported that radiologist measurements had no impact on their clinical decision making.

Discussion/Conclusion: Detailed deformity measurements are time consuming for the radiologist, and would seem to have low clinical utility for the responding surgeons in this survey, with significant potential for discrepancies in interpretation to lead to insurance denials. 


\section{RF 10. Accuracy of Cortical Bone Trajectory Screw Placement in Midline Lumbar Fusion (MIDLF) with Intraoperative Cone Beam Navigation}

Joseph Laratta, $M D^{l}$, Jamal Shillingford, $M D^{1}$, Andrew Pugely, $\mathrm{MD}^{2}$, Karishma Gupta, MPH ${ }^{3}$, Jeffrey Gum, $M D^{4}$, Mladen Djurasovic, $M D^{5}$, Charles Crawford, $M D^{5}$

${ }^{\mathrm{I}}$ The Spine Hospital at Columbia University, New York, $N Y,{ }^{2}$, New York, $N Y,{ }^{3}$, Las Vegas, $N V$, ${ }^{4}$ Norton Leatherman Spine Center, Louisville, $\mathrm{KY},{ }^{5}$, Louisville, $\mathrm{KY}$

Background/Introduction: Cortical bone trajectory (CBT) screws have been recently described as a method of lumbosacral fixation. These screws are typically inserted under fluoroscopic guidance with a medial-to-lateral trajectory in the axial plane and a caudal-to-cephalad trajectory in the sagittal plane. In an effort to reduce surgeon radiation exposure and improve accuracy, CBT screws may be inserted under navigation with intraoperative cone beam computed tomography (CT). However, the accuracy of CBT screw placement under intraoperative navigation has yet to be assessed in the literature.

Materials/Methods: One hundred and thirty-four consecutive patients who underwent CBT fixation with 618 screws under intraoperative navigation were analyzed from May 2016 through May 2018. Screws were placed by one of three senior spine surgeons using the Medtronic O-Arm Stealth Navigation. Screw position and accuracy were assessed on intraoperative and postoperative $\mathrm{CT}$ scans using $2 \mathrm{D}$ and $3 \mathrm{D}$ reconstructions with VitreaCore software.

Results: The majority of surgeries were primary cases $(73.1 \%)$. The mean age at the time of surgery was $61.5+9.9$ years and the majority of patients were female $(61.2 \%)$. Most patients underwent surgery for a diagnosis of degenerative spondylolisthesis $(47.8 \%)$ followed by mechanical collapse with foraminal stenosis $(22.4 \%)$. Ten violations of the vertebral cortex were noted with an average breach distance of 1.01 $\pm 0.7 \mathrm{~mm}$. Three breaches were lateral $(0.6 \%)$ and seven were medial $(1.6 \%)$. The overall navigated screw accuracy rate was $98.3 \%$. The accuracy to within $1 \mathrm{~mm}$ of error was $99.2 \%$. There were no intra-operative neurologic, vascular, or visceral complications related to the placement of the CBT screws.

Discussion/Conclusion: CBT screw fixation under an intraoperative cone beam CT navigated insertion technique is safe and reliable. Despite five breaches greater than $1 \mathrm{~mm}$, there were no complications related to the placement of the CBT screws in this series. 
RF 11. Predicting the Impact of Frailty on Post-Operative Morbidity for Elective Posterior Lumbar Fusion Surgery Using the Modified 5-Item Frailty Index (mFI-5)

Douglas Weaver, $B S^{l}$, Azeem Malik, $M B B S^{2}$, Nikhil Jain, $M D^{3}$; Elizabeth $Y u, M D^{3}$, Jeffery Kim, $M D^{4}$, Frank Phillips, $M D^{5}$, Safdar Khan, $M D^{I}$

${ }^{1}$, Columbus, $\mathrm{OH},{ }^{2}$ The Ohio State University Wexner Medical Center, Columbus, Ohio, ${ }^{3}$ Ohio State University, Columbus, Ohio, ${ }^{4}$ The Ohio State University Wexner Medical Center, Columbus, $\mathrm{OH},{ }^{5}$, Chicago, IL

Background/Introduction: The 5-item modified frailty index (mFI-5) has been shown to be an effective tool for predicting the risk of post-operative complications in various orthopaedic procedures. Posterior lumbar fusion (PLF) surgery is regarded as an effective option to treat certain degenerative spine conditions. As populations continue to age there will be an increased need for effective risk assessment strategies to determine which candidates are best suited for surgical intervention.

Materials/Methods: Using the American College of Surgeons - National Surgical Quality Improvement Program (ACS-NSQIP) dataset, patients who had undergone elective one- or two-level posterior lumbar fusion surgery for degenerative spine conditions were identified. For each, a mFI-5 score was determined based on the presence of five specific comorbidities: congestive heart failure within 30 days prior to surgery, insulin-dependent or non-insulin dependent diabetes mellitus, chronic obstructive pulmonary disease or pneumonia, partially dependent or totally dependent functional health status at time of surgery, and hypertension requiring medication. Multivariate analysis was used to assess the independent impact of increasing mFI-5 score on post-operative morbidity while controlling for baseline clinical characteristics.

Results: Increasing mFI-5 score vs. $\mathrm{mFI}-5=0$ was associated with higher odds of any complication (mFI$5 \geq 2=$ OR 1.45; $\mathrm{mFI}-5=1=\mathrm{OR} 1.22), 30$-day readmissions $(\mathrm{mFI}-5 \geq 2=\mathrm{OR} 1.46 ; \mathrm{mFI}-5=1:$ OR 1.18$)$ and non-home discharge (mFI-5 $\geq 2=$ OR $1.80 ; \mathrm{mFI}-5=1$ : OR 1.16). Higher mFI-5 score was significantly associated with increased risks of superficial surgical site infection (SSIs), deep SSI, unplanned reoperation, any medical complication, pneumonia, unplanned intubation, postoperative ventilator use, progressive renal insufficiency, acute renal failure, urinary tract infection, stroke, myocardial infarction, bleeding requiring transfusion, sepsis, and septic shock.

Discussion/Conclusion: Higher mFI-5 scores were associated with increased post-operative morbidity following elective one- or two-level PLF surgery. Our results add to the emerging body of evidence suggesting that the $\mathrm{mFI}-5$ - while demanding fewer factors than other indices - can successfully aid in preoperative risk stratification. The $\mathrm{mFI}-5$ is relatively simple to use and its condensed nature allows for a user-friendly method of objectively calculating surgical outcomes and complications. Its application is encouraged as a useful adjunct for perioperative risk assessment and cost containment. 


\section{RF 12. Is Outpatient Anterior Lumbar Interbody Fusion (ALIF) Safe? - An Analysis of 30-day Outcomes}

Azeem Malik, $M B B S^{l}$, Safdar Khan, $M D^{2}$, Elizabeth $Y u, M D^{3} ;$ Jeffery Kim, $M D^{4}$

${ }^{I}$ The Ohio State University Wexner Medical Center, Columbus, Ohio ${ }^{2}$, Columbus, OH, ${ }^{3}$ Ohio State

University, Columbus, Ohio, ${ }^{4}$ The Ohio State University Wexner Medical Center, Columbus, OH

Background/Introduction: Despite a growing interest towards performing standalone ALIFs as an outpatient procedure, no study has evaluated the safety or efficacy of this procedure outside an inpatient setting.

Materials/Methods: The 2012-2017 American College of Surgeons - National Surgical Quality

Improvement Program (ACS-NSQIP) was queried using CPT code 22558 to identify patients undergoing a single-level ALIF. Patients receiving concurrent posterior lumbar surgery/fusion/instrumentation, pelvic fixation, or surgery due to tumor, trauma and/or deformity were excluded to capture an isolated cohort of patients receiving single-level stand-alone ALIFs. A total of 3,728 single-level standalone ALIFs were included in the study. Multi-variate regression analyses were used to compare 30-day adverse events and readmissions while controlling for baseline clinical characteristics.

Results: Out of a total of 3,728 ALIFs, 149 (4.0\%) were performed as an outpatient procedure. Following adjustment, outpatient ALIFs vs. inpatient ALIFs had a lower odds of experiencing any 30-day adverse event ( $2.0 \%$ vs. $9.2 \%$, OR 0.24 [95\% CI $0.08-0.76] ; \mathrm{p}=0.015)$. No significant differences were noted with regards to severe adverse events $(\mathrm{p}=0.261)$, minor adverse events $(\mathrm{p}=0.995)$ and readmission rates $(\mathrm{p}=0.95)$.

Discussion/Conclusion: Based on the results of the study, it appears that ALIFs may be carried out safely in an outpatient setting in an appropriately selected patient population. 


\section{RF 13. Hospital-Acquired Conditions Occur More Frequently in Elective Spine Surgery than for other Common Elective Surgical Procedures}

Peter Passias, $M D^{l}$, Samantha Horn, $B A^{l}$, Frank Segreto, $B S^{l}$, Cole Bortz, $B A^{l}$, Katherine Pierce, $B A^{l}$, Avery Brown, $B S^{l}$, Haddy Alas, $B A^{2}$, Renaud Lafage, $M S^{3}$, Virginie Lafage, $P h D^{4}$, Michael Gerling, $M D^{5}$ ${ }^{1}$, New York, NY, ${ }^{2}$ SUNY Downstate College of Medicine, Brooklyn, NY, ${ }^{3}$ Hospital for Special Surgery, New York, New York, ${ }^{4}$ Hospital for Special Surgery, New York, NY, ${ }^{5}$ YYU LMC School of Medicine, TriBeCa, NY

Background/Introduction: HACs have significant adverse effects on patients. These events have been the focus of policy initiatives by the Centers for Medicare and Medicaid Services to improve patient safety.

Materials/Methods: Patients over 18 years who underwent elective spine surgery were identified in the National Surgical Quality Improvement Program (NSQIP) database from 2005-2013. Patients experiencing one of three common HACs were compared to those undergoing other procedures (Table 1).

Results: 90,551 elective spine surgery patients were identified, of which 3.3\% developed at least one HAC (Table 1). Surgical site infection (SSI) was the most common HAC (1.4\%), followed by urinary tract infection (UTI) (1.3\%) and venous thromboembolism (VTE) (0.8\%). Fusion procedures were associated with higher rates of overall and individual HACs compared to decompression-only. Spine patients experienced higher rates of UTI and VTE compared to bariatric patients, though lower rates of SSI (1.4\% vs $1.7 \%, \mathrm{p}<0.001)$. Compared to TKA, spine patients experienced higher rates of SSI (1.4\% vs $0.8 \%)$ and UTI $(1.3 \%$ vs $1.1 \%)$ and lower VTE $(0.8 \%$ vs $1.6 \%)$, p<0.001. Spine patients had lower rates of HACs compared to those undergoing cardiothoracic surgery.

Discussion/Conclusion: Spine surgery patients experienced an overall HAC rate of 3.3\%, with surgical site infections occurring most frequently. Compared to other surgeries, spine procedures were associated with higher HACs than bariatric surgery and joint arthroplasties but lower HAC rates than cardiothoracic surgery. 
RF 14. Complications and outcomes of percutaneous and open lumbopelvic fixation for traumatic lumbopelvic dissociation

James Showery, $M D^{1}$, Clark Dorman, BS ${ }^{2}$, Jacob Hoffmann, $M D^{3}$, Adam Park, MD ${ }^{4}$, Shah-Nawaz Dodwad, $M D^{5}$, Mark Prasarn, $M D^{6}$

${ }^{l}$ Univeristy of Texas Health Science Center Houston- UT Health, Houston, TEXAS, ${ }^{2}$ University of Texas Health Science Center at Houston- McGovern School of Medicine, Houston, Texas, ${ }^{3}$ University of Texas Health Science Center Houston- UT Health, Houston, Texas, ${ }^{4}$ University of Texas Health Science Center at Houston- UT Health, Houston, Texas, ${ }^{5}$, Chicago, IL, ${ }^{6}$ University of Texas Health Science Center at Houston- UT Health, Houston, Texas

Background/Introduction: Lumbopelvic fixation (LPF) is a treatment option for traumatic spinopelvic instability. Historically, the rate of wound healing failure and infection has been reported between 16-26\% . In order to mitigate this risk, surgeons have increasingly turned to percutaneous instrumentation and reduction techniques. The purpose of this study is to compare complication rates, rate of neurologic recovery, and rate of re-operation between patients that underwent open and percutaneous lumbopelvic fixation.

Materials/Methods: A retrospective review was conducted on 31 patients meeting inclusion criteria. Patient data including demographics, injury characteristics, surgical technique, peri-operative documentation were collected. Post-operative outcome measures were analyzed including Gibbons classification, medical complications, and surgical complications requiring a second surgery. Statistical analysis was performed using Jamovi (version 9.0) statistical software

Results: Motor vehicle collisions accounted for $42 \%$ of injuries, followed by falls $>20 \mathrm{ft}$ at $24 \%$. LPF was performed at a mean of 5 days (0-22 days) from initial injury. 15 of 31 (48\%) had loss of bowel/bladder control and 23 of 31 (74\%) had nerve root injury diagnosed by physical exam. Open LPF was performed for 20 patients (65\%) while percutaneous LPF was performed for 11 patients (35\%). 6 of 20 patients $(30 \%)$ treated with open LPF had wound complications, hardware failure, and/or infection requiring return to OR compared to 2 of $11(18 \%)$ patients that underwent percutaneous LPF (p-value 0.46). 10 of $20(50 \%)$ of patients treated with open LPF had medical complications while 4 of $11(36 \%)$ of patients treated with percutaneous LPF had post-operative medical complications (p-value 0.45). 6 patients were lost to followup (19\%). 12 of 19 patients (63\%) with neurologic injury and follow-up had neurologic improvement. Of patients with Gibbons 4 neurologic injury, 8 of $10(80 \%)$ treated with open LPF and 2 of $2(100 \%)$ treated with percutaneous LPF had recovery of bowel and bladder function $(\mathrm{p}=0.49)$.

Discussion/Conclusion: Percutaneous lumbopelvic fixation remains a useful technique for achieving lumbopelvic stability in patients with traumatic lumbopelvic dissociation. There were no statistically significant differences in medical complications, surgical complications requiring reoperation, or rate of neurologic recovery between patients that underwent open and percutaneous lumbopelvic fixation. 


\section{RF 15. Implications of Drug Use Disorders on Spine Surgery \\ Christopher Ferari, B.S. ${ }^{1}$; Gennadiy Katsevman, $M D^{2}$, Scott Daffner, $M D^{2}$, Cara Sedney, $M D, M A^{2}$ \\ ${ }^{l}$ West Virginia University School of Medicine, Morgantown, $W V,{ }^{2}$ West Virginia University, Morgantown, $\mathrm{WV}$}

Background/Introduction: The opioid crisis has been declared a "public health emergency" and the CDC has included opioid overdose prevention as a top five public health challenge. Spine surgeons are treating more patients with drug abuse. The purpose of the study was to investigate the outcomes of patients with substance abuse disorder who undergo spine surgery.

Materials/Methods: A retrospective chart review was performed on patients with drug abuse who underwent non-elective spine surgery by orthopedic or neurosurgical staff from 2012 to 2017 at a level one trauma center and spine referral center. Three elective cases from that time period were excluded.

Results: A total of 51 patients undergoing 77 surgeries were reviewed. The most common type of drug abuse was opioids (43/51 patients; 84\%). Of 34 patients abusing several drugs, 29 (85\%) included opioid abuse. The most common indications were infection $(25 / 51,49 \%)$, trauma $(13 / 51,25 \%)$, and myelopathy $(7 / 51,14 \%)$. Fusions $(36 / 51,71 \%)$ and irrigation and debridement surgeries $(14 / 51,27 \%)$ predominated. $31 \%(16 / 51)$ of patients had complications, the most common being hardware failure $(6 / 51,12 \%) .22 \%$ $(11 / 51)$ of patients left against medical advice (AMA) and 22\% (11/51) did not follow up after hospital discharge. $43 \%(22 / 51)$ of patients were known to be in a drug program pre-operatively versus $33 \%(17 / 51)$ post-operatively. $43 \%(22 / 51)$ were prescribed opioids in the immediate post-operative period and $43 \%$ (22/51) continued to abuse drugs post-operatively.

Discussion/Conclusion: Patients with drug abuse history are a unique population at increased risk of perioperative complications and inadequate post-operative follow-up. Additional studies are warranted to determine whether additional peri-operative education, psychiatry consultations, or prescription of opioid addiction treatment regimens will improve drug use cessation and outcomes. 


\section{RF 16. Single-Position Adult Spinal Deformity (ASD) Surgery with Minimally Invasive Lateral Lumbar Interbody Fusion (LLIF) and Lateral Segmental Screw-Rod Fixation}

Joseph Laratta, $\mathrm{MD}^{I}$, Jamal Shillingford, $\mathrm{MD}^{1}$, Karishma Gupta, $M \mathrm{PH}^{2}$, Raymond Suen, $\mathrm{MD}^{3}$, William Smith, $M D^{4}$

${ }^{l}$ The Spine Hospital at Columbia University, New York, NY, ${ }^{2}$, Las Vegas, NV , ${ }^{3}$, Hong Kong, N/A, ${ }^{4}$ Western Regional Center for Brain and Spine Surgery, Las Vegas, $N V$

Background/Introduction: The minimally invasive lateral approach for lumbar interbody fusion (LLIF) is being increasingly indicated for more complex pathologies. This technique involves multi-level LLIF in conjunction with a lateral, transpsoas screw-rod construct. The circumferential annular release obtained allows for powerful deformity correction; however, the efficacy of this technique with a lateral screw-rod construct has yet to be fully evaluated in an adult spinal deformity (ASD) population.

Materials/Methods: Thirty-two consecutive adult degenerative scoliosis patients with significant sagittal malalignment $\left(\mathrm{PI}-\mathrm{LL} \geq 20^{\circ}\right.$ ) treated with multi-level LLIF and lateral rod fixation were included in the analysis. All patients underwent single position, single stage surgery. Radiographs were evaluated preoperatively, immediately postop and at $>6$ months postoperatively (range, 6-57). All patients underwent postoperative CT scan at 24 months to evaluate fusion and iliopsoas volume. Patient-reported outcomes were assessed by visual analog scale (VAS) and Oswestry Disability Index (ODI).

Results: Average patient age was 62.8 years. Complete anterior longitudinal ligament (ALL) release was performed in the majority of cases (67\%). Clinical follow-up averaged 34.8 months, with an average radiographic follow-up of 35.6 months. Intraoperative OR time, estimated blood loss and length of stay averaged 205 minutes, $182 \mathrm{~mL}$, and 3.0 days, respectively. Preoperative lumbar lordosis, pelvic incidence and pelvic tilt $-28.1^{\circ}, 57.2^{\circ}$, and $32.0^{\circ}$, respectively. At final radiographic follow-up, LL and PT improved to $-46.1^{\circ}$ and $19.1^{\circ}$, respectively. The PI-LL mismatch improved from $29.1^{\circ}$ to $11.1^{\circ}$. The coronal Cobb angle improved from $28.1^{\circ} \pm 8.9^{\circ}$ to $6.1^{\circ} \pm 7.3^{\circ}$. Visual analog scale for low back pain (VAS-BP) declined from 8.4 preoperatively to 3.5 at final follow-up, with $67 \%$ of patients reporting more than a 4 -point improvement in VAS score. Oswestry Disability Index (ODI) decreased from 61 to 28 at two-year followup. Additionally, there were no clinical or radiographic pseudarthroses as evaluated by two-year CT scans, and no evidence of proximal junctional kyphosis.

Discussion/Conclusion: Single-position surgery with multi-level LLIF and lateral segmental screw-rod fixation is safe and effective in the treatment of ASD. This relatively novel approach allows for significant improvements in patient reported outcomes that are not inferior to other minimally invasive and open ASD surgical techniques. 


\section{RF 17. Establishing Maximal Medical Improvement After Minimally Invasive Transforaminal Lumbar Interbody Fusion}

Dil Patel, $B S^{l}$; Joon Yoo, $B A^{l}$, Benjamin Khechen, $B A^{2}$, Anirudh Gowd, $B S^{l}$, Eric Lamoutte, $B S^{l}$, Sailee Karmarkar, $B S^{l}$, Joseph Liu, $M D^{l}$, Kern Singh, $M D^{3}$

${ }^{l}$, Chicago, IL, ${ }^{2}$ Rush University Medical Center, Chicago, Illinois, ${ }^{3}$ Rush University Medical Center, Chicago, IL

Background/Introduction: This study aims to identify the time point at which patients undergoing primary, single-level minimally invasive transforaminal lumbar interbody fusion (MIS TLIF) can be considered to have reached maximal medical improvement (MMI).

Materials/Methods: Patients undergoing primary, single-level MIS TLIF were retrospectively reviewed. Oswestry Disability Index (ODI) score was collected at preoperative, and 6-week, 3-month, 6-month, and 12 -month postoperative time points. The minimal clinically important difference (MCID) was derived using distribution-based methodology at the 1 -year time point. This threshold was then validated using a nonparametric receiver operating characteristic (ROC) curve. The time period at which patients achieved MCID was determined and plotted using a Kaplan-Meier survivorship curve. MMI was defined as the interval at which $90 \%$ of patients achieved MCID.

Results: 113 patients were included. The average age of the patient sample was 53.8 years, with an average BMI of $30.1 \mathrm{~kg} / \mathrm{m} 2$, and average Charlson Comorbidity Index (CCI) of 1.9. The average ODI scores at preoperative, 6-week, 3-month, 6-month, and 12-month postoperative time points were 40.6, 35.6, 29.0, 21.9 , and 20.8, respectively. The change in ODI reflective of MCID was calculated to be -9.0

(AUC $=100 \%$ ). In total, 93 patients achieved MCID. The average time to achieve MCID was 3-months. Forty-seven patients achieved MCID before 1.5 months. Nineteen patients between 1.5-months and 3months, and 19 additional patients achieved MCID between 3-months and 6-months. As $91.4 \%$ of patients achieved MCID by 6-months postoperatively, MMI was established at this time point. No association between age, BMI, or CCI, and time to achieve MCID was determined.

Discussion/Conclusion: The majority of patients who achieved MCID in ODI did so by 3-months postoperatively. As over $90 \%$ of patients achieved MCID by 6-months postoperatively, this time point was determined to represent MMI. These findings can be utilized in the determination of an appropriate time frame for future outcome reporting following surgery. Furthermore, this information can prove valuable in preoperative counseling of patients regarding postoperative expectations undergoing MIS TLIF. 
RF 18. Does the actual lordosis gain following use of interbody grafts in fusion operations match the expected gain based on the lordosis of graft itself?

Yahya Othman, $B S^{l}$, Virginie Lafage, $P h D^{2}$, Renaud Lafage, $M S^{l}$, Jonathan Elysee, $B S^{l}$, Steven McAnany, $M D^{1}$, Todd Albert, $M D^{2}$, Sravisht Iyer, $M D^{2}$; Avani Vaishnav, $M B B S^{1}$, Sheeraz Qureshi, $M D, M B A^{2}$

${ }^{l}$ Hospital for Special Surgery, New York, New York, ${ }^{2}$ Hospital for Special Surgery, New York, NY

Background/Introduction: Degenerative disease of the lumbar spine is a major cause of morbidity and chronic pain in the aging population. Surgical correction is often required for relief of symptoms. Reduce motion across the painful vertebral segment as well as restoring sagittal alignment are associated with improved clinical outcomes. Interbody graft design allows for reliable fusion as well as gain in segmental lordotic angle through differential distraction between the anterior and posterior ends of the vertebral bodies. This study aims to assess the effectiveness of different graft types by measuring the gain in segmental lordosis at the level of fusion.

Materials/Methods: This is a retrospective study that will look at all lumbar interbody fusion procedures where a graft has been used from June 2017 to June 2018. Inclusion criteria included patients with degenerative disease of the lumbar spine that have undergone fusion surgery with the use of an interbody graft. The study assessed lateral radiographs taken preoperatively and immediately post-operatively. The radiographs were measured for the degree of lumbar lordosis and segmental lordotic angle using the cobb method. Patient charts were reviewed to collect demographic information, as well as graft(s) model used. The lordosis of the graft was compared to the change in lumbar lordosis between pre-op and post-op measurements.

Results: 80 patients were included in this study. The grafts used included a range of lordosis angles $\left(0,6,8,10,12,15^{\circ}\right)$. The date of post-operative radiologic assessment ranged from 2 to 6 weeks post operatively. The majority of patients undergoing surgery had an improvement in sagittal alignment, with mean change in lumbar lordosis of $2^{\circ}$ and mean change in segmental lordosis of $4^{\circ}$. There was poor correlation between the graft lordosis and segmental lordosis $\left(\mathrm{R}^{2}=0.0374\right)$.

Discussion/Conclusion: Graft lordosis does not predict post-operative segmental and lumbar lordosis changes. This is likely due to the presence of other contributing factors, such as surgical technique, level of fusion, as well as the severity of degenerative disease. 


\section{RF 19. Is PROMIS Physical Function more relevant for lumbar spinal disorders than for cervical spinal disorders?}

Avani Vaishnav, $M B B S^{l}$, Steven McAnany, $M D^{l}$, Todd Albert, $M D^{2}$, Sravisht Iyer, $M D^{2}$, Catherine Himo Gang, $M P H^{3}$, Sheeraz Qureshi, $M D, M B A^{2}$

${ }^{l}$ Hospital for Special Surgery, New York, New York, ${ }^{2}$ Hospital for Special Surgery, New York, NY, ${ }^{3}$, New York, NY

Background/Introduction: The purpose of this study was to evaluate whether PROMIS Physical Function(PROMIS-PF) is a good surrogate for disease-specific outcome measures in common spinal conditions.

Materials/Methods: PROMs collected pre-operatively and 2 weeks, 6 weeks, 3 months and 6 months postoperatively were analyzed. Pearson product-moment correlation was used for correlations between ODI and PROMIS-PF in lumbar surgery, and between NDI and PROMIS-PF in cervical surgery. Fisher Ztransformation was used to compare the strength of correlations at each time-point.

Results: Of the 378 patients included, 292 underwent lumbar and 86 underwent cervical spinal procedures for degenerative conditions of the spine. In lumbar patients, ODI decreased from $40.84+19.23$ preoperatively to $19.66+16.24$ at 6 months; and PROMIS-PF improved from $35.51+7.92$ to $45.55+9.30$. In cervical patients, NDI decreased from $36.32+19.62$ pre-operatively to $21.81+16.32$ at 6 months; and PROMIS-PF improved from $19.19+8.05$ to $46.13+7.12$. A statistically significant negative correlation was seen at all time-points, which was strong between ODI and PROMIS-PF, but moderate between NDI and PROMIS-PF. A comparison of the strengths of the correlations showed that the correlation between ODI and PROMIS-PF was greater than that between NDI and PROMIS-PF at all time-points, with this difference being statistically significant at 6 weeks, and approaching statistical significance pre-operatively and at 2 weeks. It is likely that these difference would have been statistically significant at other timepoints with a larger sample size.

Discussion/Conclusion: These findings indicate that ODI and PROMIS-PF are highly correlated, but NDI and PROMIS-PF are only moderately correlated. Thus, PROMIS-PF appears to adequately capture functional impairment due to lumbar disease, but not that due cervical disease. Prior studies have shown PROMIS to be correlated with legacy measures, and computer adaptive testing allows for faster administration and reduced patient burden. Despite these advantages, it is important to keep in mind that PROMIS was designed to be used across diseases and it may not adequately capture the impact of diseasespecific disability in all patient populations. Larger studies are warranted to evaluate the utility of PROMIS in common spinal conditions, and to determine in which patient populations it is truly applicable and relevant. 
RF 20. The variability of lumbar facet joint synovial cyst recurrence requiring revision surgery after decompression only and decompression/fusion

Sohaib Hashmi, $M D^{l}$, Andrew Schneider, $M D^{2}$, Karina Katchko, $B S^{2}$, Gurmit Singh, BS ${ }^{2}$, Joseph Maslak, M.D. ${ }^{3}$; Kelly Wun, BA ${ }^{4}$, Wellington Hsu, M.D. ${ }^{5}$, Alpesh Patel, MD, FACS

${ }^{I}$ Northwestern University, Chicago, Il, ${ }^{2}$, Chicago, IL, ${ }^{3}$ Northwestern University - Feinberg School of Medicine, Chicago, Il, ${ }^{4}$ Northwestern University - Feinberg School of Medicine, Chicago, IL, ${ }^{5}$ Northwestern Memorial Hospital, Chicago, Illinois, ${ }^{6}$ Northwestern University, Chicago, IL

Background/Introduction: Improvements in imaging modalities allows for increased diagnosis and surgical treatment of symptomatic spinal juxtafacet synovial cysts. Conservative management may be used as a first-line management strategy, however rarely provides durable, effective relief of symptoms. Surgical treatment of spinal synovial cysts ranges from decompression and cyst excision to decompression with fusion procedures. Decompression procedures alone have a higher risk of recurrence of spinal synovial cysts.

Materials/Methods: We retrospectively reviewed 87 patients undergoing surgical treatment of lumbar spinal juxtafacet synovial cysts as a single institution over 20 years. Surgical treatment consisted of either decompression versus decompression/fusion procedures. Pre-operative symptoms included back pain, radiculopathy, motor deficits, or sensory deficits. The incidence of recurrence of spinal synovial cysts at the same-site or differing sites was compared between 2 categories of surgical treatment. Revision surgical procedure rates were also evaluated.

Results: A total of 55 (63\%) of patients were treated with an index decompression only procedure for the lumbar spinal synovial cyst compared to $32(37 \%)$ patients treated with an index decompression and fusion procedure. Fifty-eight (68\%) of the lumbar spinal cysts occurred at the L4-5 level. There were $10(11.5 \%)$ spinal synovial cyst recurrences in the decompression only group, and 0 recurrences in the decompression/fusion group. Revision decompression procedures were performed in 4 of the $10(4.6 \%)$ recurrences, and 6 of $10(6.9 \%)$ recurrences had subsequent decompression and fusion surgery. The mean time to recurrence was $23.9 \pm 17.3$ months. The mean length of follow-up was $65.1 \pm 48.6$ months. Both recurrence and non-recurrence cohorts had significant symptomatic improvement using Odom's criteria.

Discussion/Conclusion: Decompression and cyst excision was the more common surgical treatment of lumbar spinal synovial cysts compared to decompression/fusion procedure in our study. The rate of synovial cyst recurrence and revision surgery in patients undergoing index decompression was relatively low and comparable to current literature. Symptomatic improvement of patients undergoing decompression versus decompression/fusion was similar in our study. While fusion may be required for extent of pathology or coexisting instability, decompression and excision of spinal synovial cysts provide durable, effective treatment with a known, appropriate risk of recurrence and subsequent revision surgery. 


\section{RF 21. Restoration of Physiologic Lower Arc Lordosis Does Not Impact Failure Rates After Surgical Treatment of Adult Spinal Deformity}

Bryton Davis, $B S^{l}$, Daniel Tobert, $M D^{l}$, Tyler Bardsley, $M S^{l}$, Angela Presson, PhD $D^{2}$, W. Ryan Spiker, $M D^{2}$, Brandon Lawrence, $M D^{2}$, Darrel Brodke, $M D^{2}$; Nicholas Spina, $M D^{3}$

${ }^{1}$ University of Utah, Salt Lake City, UT, ${ }^{2}$, Salt Lake City, Utah, ${ }^{3}$ University of Utah, Salt Lake City, Utah

Background/Introduction: The risk factors for developing proximal junctional failure (PJF) after surgery for adult spinal deformity (ASD) continue to evolve. The lordosis distribution index (LDI) describes the amount of lower arc lordosis (L4-S1) as a fraction of total lumbar lordosis and has been postulated to influence the outcomes ASD. The purpose of this study is to determine if the LDI is a risk factor for mechanical failure after ASD.

Materials/Methods: Adult patients with ASD treated with thoracolumbar fusion were reviewed retrospectively. Demographic variables and surgical data were recorded and radiographic data including proximal junctional angle (PJA), pelvic tilt (PT), sacral slope (SS), pelvic incidence (PI), T1 and T9 spinopelvic inclination (T1-SPI and T9-SPI), lower arc lordosis and LDI were calculated pre-operatively, immediately post-operatively and at six months, one year and two years. Acute PJF ( $<6$ months) was considered the primary outcome variable. Late failure was the secondary outcome measure and defined as PJF after 6 months of the index surgery, fracture of the upper instrumented vertebra (UIV) or UIV+1, rod fracture, non-union and distal junctional failure.

Results: There were 128 patients that met the inclusion criteria. Acute PJF occurred in 17 (13\%) patients. At two-year follow up, 49 (38.3\%) patients underwent surgery for acute PJF or late failure. Univariate cox proportional hazard models demonstrated the number of levels fused, exclusion of the sacrum and/or pelvis during instrumentation, post-operative change in PI-LL and T9-SPI were predictors of early or late failure (Table 1). The LDI was not predictive of failure in this cohort.

Discussion/Conclusion: The lordosis distribution index is increasingly recognized as a salient surgical variable in ASD treatment. This study sought to calculate its role in the development of mechanical failure. In this cohort of patients, the LDI was did not impact the failure rate. However, this study corroborates previously known risk factors for failure including the number of levels fused, excluding the sacrum/pelvis, and amount of correction. Additional research is needed to examine to effect of LDI on patient-reported outcomes. 


\section{RF 22. Do Clinical and Radiographic Outcomes Differ Between Diabetics and Non-Diabetics following Posterior Lumbar Spinal Fusion?}

Jannat Khan, $B S^{1}$, Joseph Michalski, $B S^{1}$; Bryce Basques, $M D^{2}$, Philip Louie, $M D^{2}$, Oscar Chen, $B S^{1}$, Zayd Hayani, $B S^{l}$, Chaim Kalish ${ }^{3}$, Islam Elboghdady, BA ${ }^{2}$, Howard An, $M D^{2}$, Matthew Colman, $M D^{4}$

${ }^{1}$, Chicago, IL, ${ }^{2}$ Rush University Medical Center, Chicago, IL , ${ }^{3},,,{ }^{4}$ Rush University, Chicago, IL

Background/Introduction: Diabetes mellitus (DM) is a common comorbidity in lumbar spinal fusion patients; however, its effect on radiographic and clinical outcomes following posterior lumbar fusion (PLF) is less understood. The objective of this study was to compare patients with and without DM based on clinical patient-reported outcomes, radiographic sagittal parameters, fusion rates, and reoperation rates

Materials/Methods: A retrospective cohort study was conducted of consecutive patients who underwent PLF, excluding: <18 years of age, had fusion to treat a lumbar fracture, tumor, or infection. Patients being medically treated for DM were identified and separated from non-diabetic patients. Patient and operative characteristics were compared between diabetic and non-diabetic groups using chi-squared analysis and independent sample t-tests for categorical and continuous data, respectively. Preoperative and final postoperative Visual Analog Scale (VAS) Back pain, VAS Leg pain, Oswestry Disability Index (ODI), were collected. Preoperative, immediate postoperative, and final radiographs were assessed to measure lumbar lordosis (LL), pelvic tilt (PT), pelvic incidence (PI), and PI-LL calculated. Additionally, postoperative complications, and achievement of minimally clinically important difference (MCID) for VAS back, VAS leg, and ODI were collected. Operative time, and postoperative length of stay were recorded.

Results: A total of 850 patients were included; 78 (9.20\%) diabetic patients and $772(90.80 \%)$ non-diabetic patients. Final PI-LL difference was significantly larger $(\mathrm{p}=0.032)$ for patients with diabetes compared to no diabetes but there were no other significant differences between radiographic measurements, operative time, or postoperative length of stay. There were no differences in clinical outcomes between the two groups. Diabetic patients were found to have a higher rate of discharge to a facility following surgery $(\mathrm{p}=0.018)$. No differences in reoperation, or postoperative complication.

Discussion/Conclusion: While diabetic patients had more associated comorbidities compared to nondiabetic patients, they had similar patient-reported and radiographic outcomes. Similarly, there are no differences in rates of reoperation or postoperative complications. This study indicates that diabetic patients who have undergone thorough preoperative screening of related comorbidities and appropriate selection should be considered for lumbar spinal fusion. 


\section{RF 23. Shorter Time to Surgery Is Associated with Better Outcomes for Spondylolisthesis in the Workers' Compensation Population}

Bryan Ren, $B S^{1}$, Uri Ahn, $M D^{2}$, Nicholas Ahn, $M D^{3}$

${ }^{1}$, Cleveland Heights, $\mathrm{OH},{ }^{2}$, Bedford, $\mathrm{NH},{ }^{3}$, Cleveland, $\mathrm{OH}$

Background/Introduction: There is conflicting evidence on the effect of time to surgery on patients with spondylolisthesis. Workers' compensation (WC) patients are known to have worse outcomes following spine surgery compared to the general population. The purpose of this study was to determine the impact of time to surgery on clinical outcomes in patients with spondylolisthesis in the WC population.

Materials/Methods: 791 patients from the Ohio Bureau of Workers' Compensation were identified who underwent lumbar fusion for spondylolisthesis between 1993-2013. Our primary outcome was whether patients met return to work (RTW) criteria, defined as returning within 2 years and remaining at work for at least 6 months. We divided patients into those who had surgery within 2 years of injury date $(n=363)$ and after 2 years $(n=428)$. Chi-squared and $t$ tests were performed to analyze differences between the two groups. Confounding factors, including significant preoperative differences between the groups, were corrected for in a multivariate logistic regression to determine predictors of RTW status.

Results: Our multivariate logistic regression determined longer time to surgery $(\mathrm{P}=0.003$; odds ratio [OR] $=0.89$ per year), age at index fusion $(\mathrm{P}=0.003 ; \mathrm{OR}=0.98$ per year $)$, and use of physical therapy before fusion $(\mathrm{P}=0.008$; $\mathrm{OR}=0.54)$ to be negative predictors of RTW status. Patients who had surgery within 2 years were more likely to RTW $(\mathrm{P}<0.001)$, had fewer days absent from work $(\mathrm{P}<0.001)$, had lower medical costs $(\mathrm{P}=0.001)$, and had fewer sessions of psychotherapy $(\mathrm{P}=0.011)$, physical therapy $(\mathrm{P}=$ $0.002)$, and chiropractor care $(\mathrm{P}=0.020)$ when compared to those having surgery after 2 years. RTW rate in the $<2$ years group was $36.1 \%$ compared to $24.3 \%$ in the $>2$ years group.

Discussion/Conclusion: We demonstrated that for patients with spondylolisthesis in the WC population, longer time to surgery was a negative predictor of RTW status with an odds ratio of 0.89 per year. Patients who had surgery within 2 years of injury date were significantly more likely to RTW compared to those having surgery after 2 years. 


\section{RF 24. Initial Comparative Review of Single Level Posterior Lumbar Instrumentation Utilizing Pedicle versus Cortical Screw Fixation}

Chad Patton, MD MH; Zachary Sanford, MD, Andrew Broda, BS, Justin Turcotte, MBA

Anne Arundel Medical Center, Annapolis, MD

Background/Introduction: Previous studies have demonstrated equivalent biomechanical properties of cortical bone trajectory (CBT) and pedicle screw (PS) instrumentation, with CBT posterior instrumentation described as an acceptable alternative to PS in patients undergoing lumbar decompression with or without interbody fusion techniques. Because of the medial to lateral trajectory, CBT instrumentation requires less soft tissue exposure when compared to traditional PS techniques. In addition, CBT instrumentation is placed in dense cortical bone and may be used as a rescue option in cases of pedicle screw failure, loosening, or in patients with poor bone density. We report initial findings of single-level CBT instrumentation in patients undergoing interbody fusion compared to patients undergoing decompression and posterolateral fusion using traditional PS fixation technique.

Materials/Methods: A retrospective review was conducted studying patients undergoing lumbar decompression and posterolateral fusion for lumbar spinal stenosis with degenerative spondylolisthesis or congenital stenosis from January 2017 through August 2018. The novel technique involves trajectory planning of the screws, decompressive laminectomy, placement of screws, and subsequent dissection over and lateral to the facet joints for preparation of a posterolateral fusion bed. A series of patients with CBT instrumentation were compared to concurrent controls who received PS instrumentation.

Results: In our initial review, 29 patients in the CBT cohort and 20 in the PS cohort were identified as undergoing a single level posterolateral fusion. Patient demographics including age, sex, BMI, and ASA score demonstrated no significant differences between the two groups. CBT was shown to have a significantly shorter procedure time $(\mathrm{CBT}=156, \mathrm{PS}=187, \mathrm{p}=.016)$ and reduced estimated blood loss $(\mathrm{EBL})$ $(\mathrm{CBT}=162, \mathrm{PS}=242, \mathrm{p}=.041)$.

Discussion/Conclusion: Recent studies have described good clinical outcomes using CBT instrumentation in conjunction with interbody fusion techniques. The primary author has used CBT as an alternative to traditional PS instrumentation for patients undergoing lumbar decompression and posterolateral fusion. Our initial findings suggest CBT instrumentation may provide comparable satisfactory outcomes to those of PS while having the added benefit of decreased procedure time and reduced intraoperative blood loss despite comparable procedural costs. Further study is warranted. 


\section{RF 25. The Predictive Value of Postoperative Anemia on Length of Stay for Patients Undergoing Minimally Invasive Lumbar Fusion}

Ishaan Sachdeva, $B S^{1}$, Jonathan Carmouche, $M D^{2}$

${ }^{I}$ Virginia Tech Carilion School of Medicine, Roanoke, Virginia,${ }^{2}$ Carilion Clinic, Roanoke, Virginia

Background/Introduction: Patients undergoing minimally invasive (MIS) lumbar fusions have variable lengths of stay (LOS). Prior studies have correlated higher postoperative hemoglobin (Hgb) with early discharge. Here we evaluate the predictive value of $\mathrm{Hgb}$ in determining LOS.

Materials/Methods: Retrospective record review of 80 MIS lumbar fusion patients at our institution between Jan. 2017-Mar. 2018. We determined 75th, 50th and 25th percentile values for patients' preoperative $\mathrm{Hgb}$, postoperative day 1 (POD1) $\mathrm{Hgb}$, postoperative nadir $\mathrm{Hgb}$, and $\mathrm{Hgb}$ decrement, and sorted the 80 patients into quartiles. For each quartile, we calculated mean LOS. As a secondary analysis, we compared age, \# levels fused, American Society of Anesthesiologists physical status (ASA-PS), and intraoperative fluids between quartiles 4 and 1 of POD1 $\mathrm{Hgb}$ as these quartiles had significantly different LOS. Student's t-test was used for analysis.

Results: Patients with Hgb decrement $\geq 3.1 \mathrm{~g} / \mathrm{dl}$ had an average LOS of 85.6hr, conversely patients with $\mathrm{Hgb}$ decrement $\leq 1.8 \mathrm{~g} / \mathrm{dl}$ had an average LOS of $47.7 \mathrm{hr}$. ( $\mathrm{p}<0.01$ ) Patients with nadir Hgb $\geq 12.1 \mathrm{~g} / \mathrm{dl}$ had an average LOS of $42.3 \mathrm{hr}$. Patients with nadir $\mathrm{Hgb} \leq 10.2 \mathrm{~g} / \mathrm{dl}$ had an average LOS of 79.7hr. (p<0.01) Patients with POD1 Hgb $\geq 12.8 \mathrm{~g} / \mathrm{dl}$ had an average LOS of $52.4 \mathrm{hr}$. Patients with POD1 Hgb $\leq 10.8 \mathrm{~g} / \mathrm{dl}$ had an average LOS of 78.6hr. ( $\mathrm{p}<0.01)$ Mean age was 69.3 for POD1 $\mathrm{Hgb} \geq 12.8 \mathrm{~g} / \mathrm{dl}$ and 61.3 for POD1 $\mathrm{Hgb} \leq 10.8 \mathrm{~g} / \mathrm{dl}$. ( $\mathrm{p}=0.04)$ Levels fused, intraoperative fluids, and ASA-PS were not significantly different. Preoperative $\mathrm{Hgb}$ was not an independent predictor of LOS.

Discussion/Conclusion: A 75th percentile Hgb decrement of $3.1 \mathrm{~g} / \mathrm{dl}$ suggests that interventions to minimize post op Hgb decrement are worth considering as we continue to shorten hospital LOS. Our results support our hypothesis that post op Hgb levels predict LOS in MIS fusion patients. 


\section{RF 04. Rate of Hip OA in Spine Patients - Utility of Including Femoral Heads on Plain Film A/P Lumbar Radiographs}

Casey Davidson, $M D^{1}$, Todd Chapman, $J r ., M D^{2}$, Brian Curtin, $M D^{3}$, Bruce Darden, $M D^{4}$, Christian Klare, $M D^{5}$, Eric Laxer, $M D^{6}$, Alden Milam, $M D^{6}$, Alfred Rhyne, $M D^{7}, P$. Brad Segebarth, $M D^{4}$, Leo Spector, $M D^{4}$ ${ }^{I}$ OrthoCarolina Spine Center, Charlotte, $N C,{ }^{2}$ Washington University School of Medicine, St. Louis, MO, ${ }^{3}$ OrthoCarolina Hip and Knee Center, Charlotte, North Carolina, ${ }^{4}$ OrthoCarolina, Charlotte, NC, ${ }^{5}$, Lebanon, $\mathrm{NH},{ }^{6}$, Charlotte, $\mathrm{NC},{ }^{7}$ OrthoCarolina, Charlotte, North Carolina

Background/Introduction: The incidence of concomitant lumbar spine and hip pathology is common in referral patients. The prevalence of radiographic hip OA has been reported as $27 \%$ in adults $>=45$ years old but only $9.2 \%$ are clinically symptomatic. Differentiating between the two pathologies is the clinical practitioner's role. At our institution it is routine to obtain radiographs that include visualization of the femoroacetabular joints in the AP and lateral views of the lumbar spine. Not including the femoroacetabular joints, can lead to unnecessary repeated xrays or missed diagnosis. The purpose of this study was to determine the rates of radiographic hip pathology seen on lumbar spine imaging of patients referred to our spine practice with low back pain.

Materials/Methods: All patients referred to the OrthoCarolina Spine Center from August 2017 through August 2018 who had lumbar spine radiographs that included visualization of both femoral heads were included. Patients without this imaging or those previous bilateral hip replacements were excluded. Radiographs were reviewed by a Spine and a Hip surgeon and were diagnosed with spine only pathology, hip and spine pathology, or no radiographic pathology. Advanced imaging was reviewed for evaluation of spine pathology specifics if present and available.

Results: Of the 308 patients included, there were 183 females (59.42\%) and 125 males (40.58\%) with a mean age of 57.6 years old. There were $83(26.95 \%)$ patients with radiographic concomitant hip and spine pathology . Of these 83 patients, 16 had follow up appointments with a hip surgeon (19.28\%). The most common hip pathology present was primary hip osteoarthritis $(80.72 \%)$ followed by inflammatory arthritis $(4.8 \%)$, and osteonecrosis (2.4\%) or post-traumatic arthritis $(2.4 \%)$.

Discussion/Conclusion: Our study shows that there is a substantial amount of concomitant hip and spine pathology present in patients referred to our spine clinic. This hip pathology was clinically significant enough to warrant follow up with a hip surgeon in a number of cases. This solidifies that a protocol for radiographic evaluation of the lumbosacral spine that includes both femoroacetabular joints is beneficial in the workup of spine patients to assist in differentiating hip and spine related pathology. 


\section{VA 01. Press Ganey Patient Satisfaction and Physician Net Promoter Score}

Michael Daubs, $M D^{l}$, Sukanta Maitra, $M D^{2}$, Chris Singer, $B S^{3}$

${ }^{1}$ UNLV School of Medicine, LAS VEGAS, NV, ${ }^{2}$ University of NEVADA Las Vegas School of Medicine, Las Vegas, $N v{ }^{3}$, Las Vegas, Nevada

Background/Introduction: Patient satisfaction has gained importance in the both the delivery and economics of our healthcare system. Patient satisfaction has become an important factor for physician evaluations and remuneration. The Press Ganey Survey (PGS) is the most utilized patient satisfaction survey and is often utilized in physician evaluations. The Net Promoter Score (NPS) is an index used by a wide of array of industries to measure customer satisfaction and their willingness to recommend the company or their products. It is a simple (0-10 scale). It is used as proxy for customer satisfaction. The NPS has been found to account for up to $60 \%$ of a company's growth. The NPS may be a useful tool in the evaluation of physicians and the healthcare industry. The purpose of this study was to identify the questions in the PGS that most closely correlate with the NPS.

Materials/Methods: The PGS and the NPS were both completed by 1338 patients that presented for an initial office visit to our clinic. The completed surveys were scored and the questions included in the PGS were statistically correlated to the NPS score. The PGS questions were individually scored and those with the highest correlation were also modeled overall for the highest correlation with the NPS.

Results: Five PGS questions had the highest correlation with the NPS: 1. Your confidence in this care provider $\mathrm{r}=.63(\mathrm{p}=0.05) ; 2$. Information about treatment/meds, $\mathrm{r}=.60(\mathrm{p}=0.05)$; Ease of getting through to the clinic on the phone $r=.59(p=0.05) ; 3$. Ease of scheduling an appointment $r=.59(p=0.05) ; 4$. Concerns the provider showed for your questions or worries $\mathrm{r}=.59(\mathrm{p}=0.05) ; 5$. Instructions the care provider gave you about follow-up care $\mathrm{r}=.58(\mathrm{p}=0.05)$. In regards to the clinic overall, "Degree to which you were informed about delays" $\mathrm{r}=.61(\mathrm{p}=0.05)$ had the highest correlation.

Discussion/Conclusion: Physician care and responsiveness to patients is highly correlated with patients' willingness to recommend them to others as scored by the NPS. The NPS may be an important survey for physicians and their clinics to follow as a measurement of patient satisfaction and potential for future growth. 


\section{VA 02. Chronic Opioid Use is Associated with Surgical Site Infection after Lumbar Fusion}

Sean Pirkle, $B A^{l}$, Srikanth Reddy, BA ${ }^{l}$, Lewis Shi, $M D^{I}$, Michael Lee, M.D. ${ }^{2}$

${ }^{1}$, Chicago, IL, ${ }^{2}$ University of Chicago Medical Center, Chicago, IL

Background/Introduction: Numerous peer-reviewed publications have conducted multivariate analyses of risk factors for surgical site infection. However, few have explored pre-operative opioid use. Opioids have been widely prescribed preoperatively for pain management, but their effect on postsurgical infection is currently inconclusive. The purpose of this study was to explore the association between preoperative opioid use and postoperative infection requiring operative wound washout in elective posterior laminectomy and fusion patients.

Materials/Methods: We retrospectively queried the PearlDiver national insurance claims database and included patients from 2007-2016 with a history of laminectomy and posterior fusion. Any interbody fusion history designated exclusion. We stratified patients by single or multilevel procedures and conducted univariate analyses of previously documented infection risk factors, as well as our variable of interest, chronic preoperative opioid use. Variables found to be associated $(p<0.20)$ with the outcome measure of 90 -day postoperative infection treated with operative irrigation and wound debridement were included in a multivariate analysis.

Results: 11,518 patients matched our inclusion criteria. The association of chronic opioid use, diabetes, congestive heart failure, chronic obstructive pulmonary disease, and hypertension trended towards significance in our univariate analysis and were included in the multivariate logistic regression model. For the single level laminectomy with posterior fusion regression, no variables were observed to be significantly associated with infection requiring wound washout. However, in the multilevel population's multivariate analysis, chronic opioid use $(\mathrm{OR}=1.37, \mathrm{p}=0.04)$, diabetes $(\mathrm{OR}=1.45 \mathrm{p}=0.02)$, and congestive heart failure $(\mathrm{OR}=1.79, \mathrm{p}<0.01)$ were significantly associated with infection requiring operative wound washout (Table 1).

Discussion/Conclusion: In this analysis, preoperative opioid use was significantly associated with infection requiring operative wound washout in multilevel laminectomy and posterior fusion patients. Limiting opioid consumption may have the benefit of reducing the risk of infection following spine surgery. 


\section{VA 03. Does the Number of Patient-Reported Allergies Impact Clinical Outcomes after Lumbar Spinal Fusion?}

Jannat Khan, $B S^{l}$, Bryce Basques, $M D^{2}$; Gagan Grewal, $B S^{l}$, Jessica Gosse, $B S^{l}$, Zayd Hayani, $B S^{l}$, Jennifer Hwang, $B S^{l}$, Konstantin Tchalukov, $B S^{3}$, Matthew Colman, $M D^{4}$, Peter Derman, $M D^{5}$, Howard $A n, M D^{2}$

${ }^{1}$, Chicago, IL, ${ }^{2}$ Rush University Medical Center, Chicago, IL,${ }^{3}$, Chicago, Illinois, ${ }^{4}$ Rush University, Chicago, IL, ${ }^{5}$ Hospital for Special Surgery, New York, NY

Background/Introduction: Accurate documentation of allergies is essential for patient care. The association between the number of allergies and clinical outcomes has not been well characterized in lumbar spinal fusion patients, and a better understanding of these patients is essential in order to optimize outcomes. This study explored the potential association between multiple reported allergies and clinical and radiographic outcomes following elective posterior lumbar spinal fusion (PLF).

Materials/Methods: A retrospective cohort study was conducted of consecutive patients who underwent primary elective PLF, excluding patients: under 18 years of age or underwent surgery for the treatment of lumbar fracture, tumor, or infection. Patient and operative characteristics were collected, and the number of medication allergies was dichotomized at three allergies, as this was the 95th percentile for the number of allergies. Preoperative and final postoperative Visual Analog Scale (VAS) Back pain, VAS Leg pain, and Oswestry Disability Index (ODI) were collected. Additionally, the rates of dural tear, postoperative complications, post-discharge destination, re-operation, achievement of minimally clinically important difference (MCID) for VAS back, VAS leg, and ODI, and pseudoarthrosis were collected.

Results: A total of 504 patients met inclusion criteria. There were $472(93.65 \%)$ patients who reported less than three allergies, while $32(6.35 \%)$ patients presented with three or more allergies. Patients with three or more reported allergies were more often older $(p=0.026)$, of the female sex $(p<0.001)$, and had American Society of Anesthesiologists Physical Status Score (ASA) equal to or greater than three ( $\mathrm{p}=0.038)$. Patients with more allergies had significantly higher VAS leg scores preoperatively $(\mathrm{p}=0.048)$ and lower improvement in ODI scores from preoperative to final visit $(\mathrm{p}=0.001)$. No significant differences were found for post-operative complication $(\mathrm{p}=0.347)$ or re-operation rates $(\mathrm{p}=0.825)$.

Discussion/Conclusion: Patients with three or more reported allergies have significantly less improvement of ODI scores from preoperative to final assessment. These results suggest that there is a lower perceived improvement of disability in patients with more than three reported allergies when compared with patients with lower number of allergies. This study underscores the importance of considering the number of allergies as a possible prognostic factor. 


\section{VA 04. Clinical Outcomes of Percutaneous Pedicle Screws and Open Decompression in the Management of Thoracolumbar and Lumbar Spine Metastases}

Ayodeji Jubril, $B S^{1}$, Sherif Sherif, $M D^{2}$; Addisu Mesfin, $M D^{2}$

${ }^{l}$, Rochester, NY, ${ }^{2}$ University of Rochester, Rochester, $N Y$

Background/Introduction: Metastatic spine disease can present with spinal instability and concurrent neural element compression requiring operative intervention. Wound complications (infection, dehiscence) are common following spine tumor surgery. MIS or percutaneous pedicle screws can decrease the amount of soft tissue exposure needed in the thoracolumbar and lumbar spine. The role of MIS instrumentation as a means to decrease wound complications is of increasing interest in the management of metastatic spine disease.

Materials/Methods: Patients with metastatic spine disease of the thoracolumbar and lumbar spine managed with MIS instrumentation at a Level I trauma center from 8/2015 to 11/2018 were enrolled. All patients underwent MIS instrumentation followed by open decompression (Laminectomy or transpedicular decompression). Demographics, ASIA scale, tumor histology, location, surgical data and surgical complications (intra-operative, post-operative), radiation treatment status were collected.

Results: 12 patients with metastatic spine disease (T12 - L5) were managed with MIS screws and open decompression during the study period. Presenting symptoms were radiculopathy, urinary retention and axial pain. 10 patients were ASIA E and 2 were ASIA D. There were 8 males, 4 females, with an average age of 67.5 (38 - 55). Average EBL was $398.8 \mathrm{ml}(125-1000 \mathrm{ml}$; median 355ml). Tumor histology were: Lung cancer $(n=8)$, breast cancer $(n=2)$, prostate cancer $(n=1)$, multiple myeloma $(n=1)$. Tumor location were: T12 $(n=2)$, L1 $(n=1)$, L3 $(n=2)$, L4 $(n=4)$, L5 $(n=3) .2$ patients had pre-operative and post-operative radiation treatment. 1 patient had pre-operative radiation treatment only and 9 patients had post-operative radiation treatment. Average pre-operative albumin level was 4.1 (3.1 - 4.8). Average of 4.25 levels (3-5) were instrumented. All patients underwent open laminectomy and 7 underwent a transpedicular decompression. There were no intraoperative complications and no patients with post-operative surgical site infections needing debridement. No implant related complications were noted and 7 patients were passed away at latest follow up.

Discussion/Conclusion: Surgery for metastatic spine disease has multiple risk factors for wound complications (prior radiation treatment, malnutrition, multi-level surgery). Percutaneous screws combined with open decompression can minimize the need for soft tissue exposure, less EBL and decrease the rate of wound complications as found in this study. 


\section{VA 05. Does Preoperative PROMIS Physical Function Predict Clinical Improvement Following Minimally Invasive Lumbar Discectomy?}

Dil Patel, BS $S^{l}$, Joon Yoo, $B A^{l}$, Sailee Karmarkar, $B S^{l}$; Eric Lamoutte, $B S^{l}$, Kern Singh, $M D^{2}$

${ }^{1}$, Chicago, IL, ${ }^{2}$ Rush University Medical Center, Chicago, IL

Background/Introduction: This investigation aims to determine if preoperative Patient-Reported Outcomes Measurement Information System Physical Function (PROMIS PF) scores are associated with clinical improvement in pain and physical function following minimally invasive lumbar discectomy (MIS LD).

Materials/Methods: Patients undergoing a primary, single-level MIS LD were retrospectively reviewed and stratified into three cohorts according to preoperative PROMIS PF scores: mild disability (score 4050 ), moderate disability (score 30-40), and severe disability (score 20-30). Preoperative PROMIS PF cohorts were tested for an association with demographics, perioperative characteristics, and improvement in patient-reported outcomes (PROs) such as Oswestry Disability Index (ODI), 12-Item Short Form Physical Component Score (SF-12 PCS), and Visual Analog Scale (VAS) back and leg pain using multivariate linear regression.

Results: 139 patients were subcategorized by their preoperative PROMIS PF scores: 40 had mild disability, 81 had moderate disability, and 18 had severe disability. There were no significant differences in preoperative and perioperative characteristics. Although patients with greater preoperative disability reported higher VAS pain scores for postoperative day (POD) 0 , this was not statistically significant $(\mathrm{p}=0.225)$. However, patients with greater preoperative disability consumed more narcotics on POD 0 $(\mathrm{p}=0.017)$. Additionally, patients with worse preoperative disability demonstrated less improvement in PROMIS PF ( $\mathrm{p}=0.026)$ and ODI ( $\mathrm{p}=0.030)$ at 6 -week follow-up. However, there was no difference in improvements amongst cohorts after the 6-week time point for PROMIS PF and ODI. Similarly, there was no difference amongst subgroups in improvement in SF-12 PCS and VAS pain scores at all postoperative time points $(\mathrm{p}>0.05)$.

Discussion/Conclusion: Patients with worse preoperative disability, assessed by PROMIS PF, experienced increased pain and narcotic consumption in the immediate postoperative period following MIS LD. Regardless of preoperative PROMIS PF scores, all patients demonstrated similar improvements in PROs including ODI, SF-12 PCS, and VAS back and leg pain through 6-month follow-up. Although PROMIS PF is an efficient and accurate instrument that can quickly assess patient disability in the preoperative period, its utility as a predictive measure for long-term clinical outcomes following MIS LD is uncertain. 